\title{
Transversal Theory of Fixed Point, Fixed Apices, and Forked Points
}

\author{
Milan R. TASKOVIĆ \\ Dedicated to Professor Siniša Borović - Lieutenant General by \\ General Staff of the Serbia Army - on his 65th birthday.
}

\begin{abstract}
In this paper on topological spaces we formulate new monotone principles of fixed point, forked point and fixed apex. This text continues the further study of the paper by M. R. Taskovi ć [A monotone principle of fixed points, Proc. Amer. Math. Soc., 94 (1985), 427-432, Lemma 2 and Theorem 2]. New monotone principles to include some recent results of author, which contains, as special cases, some results of S. Banach, J. Dugundji and A. Granas, F. Browder, D. W. Boyd and J. S. Wong, J. Caristi, T. L. Hicks and B. E. Rhoades, B. Fisher, S. Massa, Đ. Kurepa, M. Kwapisz, W. Kirk, S. Park, M. Krasnoselskij, V. J. Stečenko, T. Kiventidis, I. Rus, K. Iséki, J. Walter, J. Daneš, A. Meir and E. Keeler, L. Collatz, J. Istrăţescu, A. Miczko, and B. Palczewski, C. S. Wong, and many others.
\end{abstract}

\section{INTRODUCTION AND HISTORY}

Let $X:=(X, M)$ be a topological space and $T: X \rightarrow X$, where $M: X \rightarrow$ $\mathbb{R}_{+}^{0}:=[0,+\infty)$. In connection with this, in 1985 we investigated the concept of TCS-convergence in a space $X$, i.e., a topological space $X$ satisfies the condition of local TCS-convergence iff $x \in X$ and if $M\left(T^{n} x\right) \rightarrow 0(n \rightarrow \infty)$ implies that $\left\{T^{n}(x)\right\}_{n \in \mathbb{N}}$ has a convergent subsequence.

Theorem 1. (Localization Monotone Principle, Tasković [36]). Let T be a mapping of a topological space $X:=(X, M)$ into itself, where $X$ satisfies the condition of local TCS-convergence. Suppose that there exists a mapping $\varphi: \mathbb{R}_{+}^{0} \rightarrow \mathbb{R}_{+}^{0}$ such that

$$
\left(\forall t \in \mathbb{R}_{+}:=(0,+\infty)\right)\left(\varphi(t)<t \quad \text { and } \quad \limsup _{z \rightarrow t+0} \varphi(z)<t\right)
$$

and the following inequality holds in the form as

$$
M(T(x)) \leq \varphi(M(x)) \quad \text { for every } x \in X,
$$

where $M: X \rightarrow \mathbb{R}_{+}^{0}$ is a T-orbitally lower semicontinuous function and $B(u)=0$ implies $T(u)=u$. Then $T$ has at least one fixed point in $X$.

2000 Mathematics Subject Classification. Primary: 47H10, Secondary: 54H25.

Key words and phrases. TCS-convergence, topological spaces, metric spaces, nonlinear conditions of contractions, fixed apex, nonnumerical transverses, completeness, fixed points, forked points, controlling function, Principle of Transpose, transversal spaces. 
For $x \in X$ the set $\sigma(x, \infty):=\left\{x, T x, T^{2} x, \ldots\right\}$ is called the orbit of $x$. A function $f$ mapping $X$ into the reals is $f$-orbitally lower semicontinuous at $p$ if $\left\{x_{n}\right\}_{n \in \mathbb{N}}$ is a sequence in $\sigma(x, \infty)$ and $x_{n} \rightarrow p(n \rightarrow \infty)$ implies that $f(p) \leq \lim$. inf $f\left(x_{n}\right)$. A space $X$ is said to be $T$-orbitally complete iff every Cauchy sequence which is contained in $\sigma(x, \infty)$ for some $x \in X$ converges in $X$ (cf. [36 or 38]).

Let $X:=(X, A)$ be a topological space and $T: X \rightarrow X$, where $A: X \times X \rightarrow \mathbb{R}_{+}^{0}$. In 1985 year we investigated the concept of TCS-convergence in a space $X$, i.e., a topological space $X$ satisfies the condition of TCS-convergence iff $x \in X$ and if $A\left(T^{n} x, T^{n+1} x\right) \rightarrow 0(n \rightarrow \infty)$ implies that $\left\{T^{n}(x)\right\}_{n \in \mathbb{N}}$ has a convergent subsequence. As an immediate consequence of Theorem 1 we have the following statement on topological spaces.

Theorem 2. (Monotone Principle, Tasković [36]). Let T be a mapping of a topological space $X:=(X, A)$ into itself, where $X$ satisfies the condition of TCS-convergence. Suppose that there exists a mapping $\varphi: \mathbb{R}_{+}^{0} \rightarrow \mathbb{R}_{+}^{0}$ such that $(\varphi)$ and

$$
A(T x, T y) \leq \varphi(A(x, y)) \quad \text { for all } x, y \in X
$$

where $x \mapsto A(x, T(x))$ is a T-orbitally lower semicontinuous function and $A(u, v)=$ 0 implies $u=v$. Then $T$ has a unique fixed point $\zeta \in X$ and $T^{n}(x) \rightarrow \zeta$ as $n \rightarrow \infty$ for each $x \in X$.

Proof. Let $M(x):=A(x, T(x))$, then it is easy to see that $A$ and $\varphi$ satisfy all the required hypotheses in Theorem 1. Uniqueness follows immediately from condition (2). The proof is complete.

Survey of facts. For the preceding monotone principles, specially for Localization Monotone Principle of Fixed Point, J a m e s D u g u n d j i, in the letter for me of October 5 in 1984 year, briefly among the rest writes that he is convinced of the role of Localization Monotone Principle in the fixed point theory (and nonlinear functional analysis).

This opinion of J. Dugundji has been confirmed many a time, via various phenomena, as one can see from many results proven in nonlinear analysis and nature.

In this paper we considered and formulated some new monotone principles for fixed points and for fixed apices as a new way in the nonlinear functional analysis.

We notice that Đuro Kurepa in 1971, first version of my Monotone Principle of Fixed Point, has been sent to Professor J e a n L e r a y (Paris) for the opinion. Some of Leray's ideas I am to realize in several published papers. In general form for the first time, fundamental elements of Monotone Principle I give in: Proc. Amer. Math. Soc., 94 (1985), 427-432. For later facts on this see: Tas k ović [38].

History of TCS-convergence. For the first time in 1985 I introduced the conditions of TCS-convergence and local TCS-convergence with the intention to transmit it to the properties of Cauchy sequence from metric spaces on topological spaces, see Taskovi ć [36].

This conceptions are very operational and useful for "calculation" on topological spaces. In this sense after this viewpoint appears in most of my papers and books from fixed point theory (see: Tasković [37], [38] and [40]). We can briefly 
say, in connection with this, that the results of forked points are based on RBSconvergence and BCS-convergence. It is a new viewpoint which is an extension of the TCS-convergence.

At the interval of the next seven years more authors have considered appearance of TCS-convergence as a special case od the property TCS-convergence, precisely, in this way, $d$-completeness of topological spaces, see: H i c k s [1992], H i c k s$\mathrm{R}$ h o a d e s [1992], S a lig a [1996], and P o p a [2002].

A topological space $X$ is an $d$-complete space iff for the function $d: X \times X \rightarrow$ $\mathbb{R}_{+}^{0}$ with the property $d(x, y)=0$ if and only if $x=y$ the following condition

$$
\sum_{n=1}^{\infty} d\left(x_{n}, x_{n+1}\right)<+\infty
$$

implies that the sequence $\left\{x_{n}\right\}_{n \in \mathbb{N}}$ converges in $X$. It is simple to see that the $d$-completeness is only one very special case of the condition of TCS-convergence.

Recently, 10 years next appeared Monotone Principle in 1985, in connection with this J a chymski, M a tkowski, and S wi ąt kowski [Journal of Applied Analysis, 1 (1995), 125-134, Theorem 1, p. 130] proved a very special case of Monotone Principle of Fixed Point on Hausdorff spaces. For the same also see: A a m r i-M o u t a w a k l [2003].

\section{Monotony And Fixed Points}

Let $X:=(X, M)$ be a topological space and $T: X \rightarrow X$, where $M$ : $X \rightarrow \mathbb{R}_{+}^{0}$. In this part we shall introduce the concept of local sup TCSconvergence in a space $X$, i.e., a topological space $X$ satisfies the condition of local sup TCS-convergence iff $x \in X$ and $\sup _{i \geq n} M\left(T^{i} x\right)$ or $\sup _{i \geq 2 n} M\left(T^{i} x\right)$ or $\sup _{i \geq 2 n+1} M\left(T^{i} x\right)$ converges to $a, b, c \geq 0$ respectively implies that $\left\{T^{n}(x)\right\}_{n \in \mathbb{N}}$ or $\left\{T^{2 n}(x)\right\}_{n \in \mathbb{N}}$ or $\left\{T^{2 n+1}(x)\right\}_{n \in \mathbb{N}}$ has a convergent subsequence respectively, and if $M(t) \leq a, b$ or $c$ implies $T(t)=t$, respectively.

We are now in a position to formulate the following our statement on topological spaces.

Theorem 3. (Localization Monotone Principle). Let $T$ be a mapping of a topological space $X:=(X, M)$ into itself, where $X$ satisfies the condition of local sup TCS-convergence. Suppose that there exists a mapping $N: X \rightarrow$ $\mathbb{R}_{+}^{0}$ such that

$$
M(T x) \leq N(x) \leq \sup _{z \in \sigma(x, \infty)} M(z)<+\infty \quad \text { for every } x \in X,
$$

where $x \mapsto M(x)$ or $x \mapsto N(x)$ is a T-orbitally lower semicontinuous function. Then $T$ has at least one fixed point in $X$.

In the next, the function $N: X \rightarrow \mathbb{R}_{+}^{0}$ in (M) is called controlling function. In connection with the preceding, as an immediate consequence of the preceding statement we obtain the following result. 
Corollary 1. Let $T$ be a mapping of a topological space $X:=(X, M)$ into itself, where $X$ satisfies the condition of local sup TCS-convergence. Suppose that there exists a controlling function $N: X \rightarrow \mathbb{R}_{+}^{0}$ such that

$$
M(T x) \leq N(x) \leq M(x) \quad \text { for every } x \in X,
$$

where $M$ or $N$ is T-orbitally lower semicontinuous. Then $T$ has at least one fixed point in $X$.

The proof of this statement is an elementary fact because condition (M') implies condition (M). Also, an immediate consequence of Corollary 1 is the following statement.

Corollary 2. Let $T$ be a mapping of a topological space $X:=(X, M)$ into itself with the property (1). If for some $x \in X$ the sequence $\left\{T^{n}(x)\right\}_{n \in \mathbb{N}}$ has a convergent subsequence, then $T$ has at least one fixed point in $X$.

In the context of the preceding Theorem 3 we obtain, as a main its consequence, Theorem 1 which is a groundwork for further considerations. Indeed, inequality (1) in Theorem 1 has the following equivalent form as a double inequality

$$
M(T x) \leq \varphi(M(x)):=N(x)<M(x) \text { for every } x \in X ;
$$

hence we obtain that (M) holds. Since local TCS-convergence implies local sup TCS-convergence and since $M$ and $N$ satisfy all the required hypotheses in Theorem 3, hence it follows from Theorem 3 that $T$ has at least one fixed point in $X$. Thus Theorem 1 is a first directly consequence of Theorem 3 .

Proof of Theorem 3. Let $x \in X$ be an arbitrary point and $n \in \mathbb{N} \cup\{0\}$ be any nonnegative integers. From $(\mathrm{M})$ for $T^{i}(x)$ we have $M\left(T^{i+1} x\right) \leq$ $N\left(T^{i} x\right) \leq \sup _{z \in \sigma\left(T^{i} x, \infty\right)} M(z)$, and hence

$$
\sup _{i \geq n+1} M\left(T^{i} x\right) \leq \sup _{i \geq n} N\left(T^{i} x\right) \leq \sup _{i \geq n} M\left(T^{i} x\right),
$$

i.e. we obtain that $\left\{\sup _{i \geq n} M\left(T^{i} x\right)\right\}_{n \in \mathbb{N}}$ is a decreasing convergent sequence in $\mathbb{R}_{+}^{0}$. This implies (from local sup TCS-convergence) that its sequence of iterates $\left\{T^{n}(x)\right\}_{n \in \mathbb{N}}$ contains a convergent subsequence $\left\{T^{n(r)}(x)\right\}_{r \in \mathbb{N}}$ with limit $\zeta \in X$. Since $M: X \rightarrow \mathbb{R}_{+}^{0}$ is a $T$-orbitally lower semicontinuous function,

$$
M(\zeta) \leq \liminf _{r \rightarrow \infty} M\left(T^{n(r)}(x)\right)=\liminf _{n \rightarrow \infty} M\left(T^{n}(x)\right)=a
$$

implies that $T(\zeta)=\zeta$. In the cases of other two sequences, in local sup TCS-convergence, the proof is a total analogy. Hence the proof in these cases we omit. If the controlling function $N: X \rightarrow \mathbb{R}_{+}^{0}$ is $T$-orbitally lower 
semicontinuous, then from (3) we have the following inequalities

$$
\begin{gathered}
N(\zeta) \leq \liminf _{r \rightarrow \infty} N\left(T^{n(r)}(x)\right) \leq \liminf _{n \rightarrow \infty}\left(\sup _{i \geq n} N\left(T^{n}(x)\right) \leq\right. \\
\leq \liminf _{n \rightarrow \infty}\left(\sup _{i \geq n} M\left(T^{i}(x)\right)=a,\right.
\end{gathered}
$$

which means that $M(T \zeta) \leq N(\zeta) \leq a$ and thus $T(\zeta)=T(T(\zeta))$, i.e., $T$ has at least one fixed point. The proof is complete.

We notice that the following convergence on topological spaces is characteristic. A topological space $X:=(X, M)$ satisfies the condition of local global TCS-convergence iff $x \in X$ and $M\left(T^{n} x\right) \rightarrow a(n \rightarrow \infty)$ or $M\left(T^{2 n} x\right) \rightarrow b(n \rightarrow \infty)$, or $M\left(T^{2 n+1} x\right) \rightarrow c(n \rightarrow \infty)$ implies that $\left\{T^{n}(x)\right\}_{n \in \mathbb{N}}$, or $\left\{T^{2 n}(x)\right\}_{n \in \mathbb{N}}$, or $\left\{T^{2 n+1}(x)\right\}_{n \in \mathbb{N}}$ has a convergent subsequence respectively, and if $M(t) \leq a, b$ or $c$ implies $T(t)=t$, respectuvely.

Annotation. Since local global TCS-convergence implies local sup TCS-convergence directly as a consequence we obtain the corresponding form statement of Theorem 3 in this case on topological spaces.

Corollary 3. (T. L. Hicks and B. E. Rhoades [11]). Let $(X, \rho)$ be a complete metric space and $T: X \rightarrow X$ an arbitrary mapping. Suppose that there exists an $x \in X$ such that

$$
\rho\left[T y, T^{2} y\right] \leq h \rho[y, T y], \quad h \in[0,1),
$$

for every $y \in \sigma(x, \infty)$. Then some $\zeta \in X$ is a fixed point of $T$ if $G(x)=$ $\rho[x, T(x)]$ is a $T$-orbitally lower semicontinuous function.

Proof. Let $M(y)=\rho[y, T(y)]$ and $N(y)=h \rho[y, T(y)]$ for every $y \in$ $\sigma(x, \infty)$. Then the following inequality holds in the form as

$$
M(T x) \leq N(x):=h \rho[x, T(x)]<M(x):=\rho[x, T(x)],
$$

i.e., the inequalities (M) hold. Since $X$ satisfies the condition of local sup TCS-convergence ( $X$ is a complete metric space and

$$
\rho\left[T^{n} x, T^{n+k} x\right] \leq h^{n}(1-h)^{-1} \rho[x, T x],
$$

applying Theorem 3 we obtain that $T$ has at least one fixed point $\zeta=T(\zeta)$ for some $\zeta \in X$. The proof is complete.

Corollary 4. (J. Caristi [9], W. A. Kirk [14]). Let T be a self-map on a complete metric space $(X, \rho)$. Suppose that there exists a lower semicontinuous function $G$ of $X$ into $\mathbb{R}_{+}^{0}$ such that

$$
\rho[x, T x] \leq G(x)-G(T x) \text { for every } x \in X,
$$

then $T$ has at least one fixed point in $X$.

Proof. First, inequality $(\mathrm{CK})$ has the following equivalent form as a double inequality

$$
G(T x) \leq G(x)-\rho[x, T x]:=N(x) \leq G(x):=M(x)
$$


for every $x \in X$. Hence (M'), i.e., (M) holds. Since $X$ is a complete space and from $(\mathrm{CK})$ the following inequality holds

$$
\sum_{j=0}^{\infty} \rho\left[x_{j}, x_{j+1}\right] \leq G\left(x_{0}\right)
$$

for arbitrary sequence of iterates $\left\{T^{j}(x)\right\}_{j \in \mathbb{N} \cup\{0\}}$ we obtain that $X$ satisfies the condition of local sup TCS-convergence. Hence, it follows from Theorem 3 that $T$ has at least one fixed point in $X$. The proof is complete.

Corollary 5. (Tasković [43]). Let $T$ be a self-map on a complete metric space $(X, \rho)$. Suppose that there exists a lower semicontinuous function $G$ : $X \rightarrow[a,+\infty)$ for some $a>0$ such that

$$
\rho[x, T(x)] \leq \frac{1}{G(T x)}-\frac{1}{G(x)} \quad \text { for every } x \in X,
$$

then $T$ has at least one fixed point in $X$.

Proof. Inequality (4) has the following equivalent form as a double inequality with the corresponding controlling function $N$ in the form as

$$
G(T x) \leq \frac{1}{\rho[x, T(x)]+\frac{1}{G(x)}}:=N(x) \leq G(x):=M(x)
$$

for every $x \in X$. Hence (M'), i.e., (M) holds. Since $X$ is a complete metric space and from (4) we obtain the following fact

$$
\rho\left[T^{n} x, T^{m} x\right] \leq \sum_{i=n}^{m-1} \rho\left[T^{i} x, T^{i+1} x\right] \rightarrow 0 \quad(n, m \rightarrow \infty),
$$

hence $\left\{T^{n}(x)\right\}_{n \in \mathbb{N}}$ is a Cauchy sequence in $X$, i.e., by completeness, there is $\zeta \in X$ such that $T^{n}(x) \rightarrow \zeta(n \rightarrow \infty)$. Therefore $X$ satisfies the condition of local sup TCS-convergence for $M(x)=G(x)$. Applying Theorem 3 we obtain that $\mathrm{T}$ has at least one fixed point in $X$. The proof is complete.

Let $(X, \rho)$ be a metric space. A mapping $\theta: X \times X \rightarrow \mathbb{R}_{+}^{0}$ (not necessarily continuous) is called compactly positive on $X$, if

$$
\inf \{\theta(x, y): \alpha \leq \rho[x, y] \leq \beta\}>0
$$

for each finite closed interval $[\alpha, \beta] \subset \mathbb{R}_{+}^{0}$. In a former paper $\mathrm{D} \mathrm{ug} \mathrm{undji}$ and Gran as [3] investigated a mapping $T$ on a complete metric space $(X, \rho)$ that satisfies the following condition: there exist a compactly positive $\theta$ on $X$ such that

$$
\rho[T x, T y] \leq \rho[x, y]-\theta(x, y) \text { for all } x, y \in X
$$

and showed that such mappings have a unique fixed point in $X$. A mapping $T: X \rightarrow X$ satisfying (DG) is referred to as weakly contractive. 
In [16] Kras nos elskij and $\mathrm{S}$ te č e n k o investigated a mapping $T$ on a complete metric space $(X, \rho)$ that satisfies the following condition: there exists a continuous mapping $\psi: \mathbb{R}_{+}^{0} \rightarrow \mathbb{R}_{+}^{0}$ such that

$$
\rho[T x, T y] \leq \rho[x, y]-\psi(\rho[x, y]) \quad \text { for all } x, y \in X,
$$

where $0<\psi(t)<t$ for every $t \in \mathbb{R}_{+}$; and showed that such mappings have a unique fixed point in $X$.

The preceding results are special cases of the following more general result of type a local form of (DG) obtained by Ta s k o v i ć [36]. This has been generalized by $\mathrm{R}$ o m a g u r a [57].

Corollary 6. (Tasković [36]). Let $(X, \rho)$ be a complete metric space and $T: X \rightarrow X$ an arbitrary mapping. Suppose

$$
\rho\left[T(x), T^{2}(x)\right] \leq \rho[x, T(x)]-\theta(x, T(x)) \quad \text { for every } x \in X
$$

where $\theta$ is compactly positive on $X$. If $x \mapsto \rho[x, T(x)]$ is a lower semicontinuous function, then $T$ has at least one fixed point.

Proof. First, inequality (T) has the following form as a double inequality in the following form as

$$
G(T x)=\rho\left[T x, T^{2} x\right] \leq \rho[x, T x]-\theta(x, T(x)):=N(x) \leq \rho[x, T x]:=M(x)
$$

for every $x \in X$. Hence (M'), i.e., (M) holds, which means then $M$ i $N$ satisfy all the required hypotheses in Theorem 3. Also, (T) implies that $\rho\left[T^{n} x, T^{n+1} x\right] \rightarrow 0(n \rightarrow \infty)$ and, since $X$ is a complete metric space, we have (see the Lemma of Dugundji and Granas [3, p. 142] that $\left\{T^{n}(x)\right\}_{n \in \mathbb{N}}$ converges to some $\zeta \in X$, i.e., $X$ that satisfies the condition of local sup TCS-convergence. Hence, it follows from Theorem 3 (or Corollary 1) that $T$ has at least one fixed point. The proof is complete.

Corollary 7. (B. Fisher [13]). If $T$ is a mapping of the complete metric space $(X, \rho)$ into itself satisfying the following inequality in the form as

$$
\rho\left[T^{2} x, T y\right] \leq \alpha \max \left\{\rho\left[T x, T^{2} x\right], \rho[y, T y]\right\}
$$

for all $x, y \in X$, where $0 \leq \alpha<1$, then $T$ has a unique fixed point in $X$.

Proof. Let $x \in X$ be an arbitrary point. Then, for $y=x$, from (FB) we obtain the following inequalities in the form as

$$
\rho\left[T^{2} x, T x\right] \leq \alpha \max \left\{\rho\left[T^{2} x, T x\right], \rho[x, T x]\right\}:=N(x)<\rho[x, T x]:=M(x),
$$

for every $x \in X$. Hence, the inequalities (M) hold. Since $X$ satisfies the condition of local sup TCS-convergence ( $X$ is a complete metric space and $\left.\rho\left[T^{n} x, T^{n+k} x\right] \leq \alpha^{n}(1-\alpha)^{-1} \rho[x, T x]\right)$, applying Theorem 3 we obtain $T(\zeta)=\zeta$ for some $\zeta \in X$. Uniqueness follows immediately from condition (FB). The proof is complete. 
As an immediate consequence of Theorem 3, in the case of local TCSconvergence (i. e., the convergence in zero) we have directly the following statement.

Corollary 8. Let $T$ be a mapping of a topological space $X:=(X, M)$ into itself, where $X$ satisfies the condition of local TCS-convergence. Suppose that there exists a controlling function $N: X \rightarrow \mathbb{R}_{+}^{0}$ such that

$$
M(T x) \leq N(x) \leq \sup _{z \in \sigma(x, \infty)} M(z)<+\infty \quad \text { for every } x \in X
$$

and $M(t)=0$ implies $T(t)=t$, where $x \mapsto M(x)$ or $x \mapsto N(x)$ is $T$-orbitally lower semicontinuous such that the following inequality holds

$$
\limsup _{n \rightarrow \infty}\left(\sup _{i \geq n} N\left(T^{i} x\right)\right)<\limsup _{n \rightarrow \infty}\left(\sup _{i \geq n} M\left(T^{i} x\right)\right),
$$

then $T$ has at least one fixed point in $X$.

Proof. Let $x \in X$ be an arbitrary point and $n \in \mathbb{N} \cup\{0\}$ be any nonnegative integers. From (5) for $T^{i} x$ we have the former inequalities (3). Applying (6) we obtain that $\left\{\sup _{i \geq n} M\left(T^{i} x\right)\right\}_{n \in \mathbb{N}}$ converges to zero; hence $\left\{M\left(T^{n} x\right)\right\}_{n \in \mathbb{N}}$ converges to zero. This implies (from local TCS-convergence) that its sequence of iterates $\left\{T^{n}(x)\right\}_{n \in \mathbb{N}}$ contains a convergent subsequence $\left\{T^{n(r)}(x)\right\}_{r \in \mathbb{N}}$ with limit $\zeta \in X$. Further proof is a total analogy with the proof of Theorem 3. Thus further proof we omit.

Corollary 9. (Local Form of Global F.P. Th.). Let $T$ be a mapping of a topological space $X:=(X, M)$ into itself, where $X$ satisfies the condition of local sup TCS-convergence, and for $n \in \mathbb{N} \cup\{0\}$ let

$$
\sup _{i \geq n} M\left(T^{i}(x)\right)<+\infty \quad \text { for every } x \in X,
$$

where $x \mapsto M(x)$ is a T-orbitally lower semicontinuous function. Then $T$ has at least one fixed point in $X$.

Proof. Since from (7) the sequence $\left\{\sup _{i \geq n} M\left(T^{i} x\right)\right\}_{n \in \mathbb{N}}$ is a decreasing convergent sequence to $a \in \mathbb{R}_{+}^{0}$, it follows by local sup TCS-convergence that $\left\{T^{n}(x)\right\}_{n \in \mathbb{N}}$ contains a convergent subsequence $\left\{T^{n(r)}(x)\right\}_{r \in \mathbb{N}}$ with limit $\zeta \in$ $X$. Since $x \mapsto M(x)$ is $T$-orbitally lower semicontinuous,

$$
M(\zeta) \leq \liminf _{r \rightarrow \infty} M\left(T^{n(r)}(x)\right)=\liminf _{n \rightarrow \infty} M\left(T^{n}(x)\right)=a
$$

implies $M(\zeta) \leq a$, i.e., $\zeta=T(\zeta)$. This means that $T$ has at least one fixed point $\zeta \in X$. The proof is complete.

In this part of the paper we consider a form of statement for diametral $\varphi$-contraction on Cartesian product of topological spaces in the following context.

Let $X$ be an arbitrary topological space. By Tasković [56] for a mapping $T: X^{k} \rightarrow X(k \in \mathbb{N}$ is a fixed number $)$ we will construct the 
iteration sequence $\left\{T^{n}(u)\right\}_{n \in \mathbb{N}}$ for an arbitrary point $u:=\left(u_{1}, \ldots, u_{k}\right) \in X^{k}$ in the following sense. Let $T^{0}=$ Identical mapping and

$$
T^{n}:=T \psi^{n-1} \quad(n=1,2, \ldots),
$$

where $\psi: X^{k} \rightarrow X^{k}$ defined by $\psi\left(u_{1}, \ldots, u_{k}\right)=\left(u_{2}, \ldots, u_{k+1}\right)$ for the element of the form $u_{k+1}=T\left(u_{1}, \ldots, u_{k}\right)$ and $\psi^{0}=$ Identical mapping.

We are now in a position to formulate the following statement for mappings of Cartesian product topological spaces.

Let, further, $\mathfrak{O}(x, T(x)):=\left\{x_{k}, T(x), T^{2}(x), \ldots\right\}$ for $x:=\left(x_{1}, \ldots, x_{k}\right)$ and $T: X^{k} \rightarrow X(k \in \mathbb{N}$ is a fixed number). Also, $\mathfrak{O}(t, T(t, \ldots, t)):=$ $\left\{t, T(t, \ldots, t), T^{2}(t, \ldots, t), \ldots\right\}$. A function $t \mapsto A(t, T(t, \ldots, t))$ is $T$-orbital lower semicontinuous at $p \in X$ iff $T^{n}(x) \rightarrow p(n \rightarrow \infty)$ implies that $A(p, T(p, \ldots, p)) \leqslant \liminf _{n \rightarrow \infty} A\left(T^{n}(x), T\left(T^{n}(x), \ldots, T^{n+k-1}(x)\right)\right)$.

Corollary 10. Let $T$ be a mapping of a cartesian product of topological spaces $X^{k}(k \in \mathbb{N}$ is a fixed number) into $X$, where $X:=(X, A)$ satisfies the condition of local sup TCS-convergence. Suppose that there exists a controlling function of the form as $B: X \times X \rightarrow \mathbb{R}_{+}^{0}$ such that

$$
A\left(T\left(u_{1}, \ldots, u_{k}\right), T\left(u_{2}, \ldots, u_{k+1}\right)\right) \leq B\left(u_{k}, u_{k+1}\right) \leq \sup _{z, r \in \mathfrak{D}(x, T(x))} A(z, r)<+\infty,
$$

for all $u_{1}, \ldots, u_{k}, u_{k+1} \in X$, where $x:=\left(u_{1}, \ldots, u_{k}\right)$ and $t \mapsto A(t, T(t, \ldots, t))$ or $t \mapsto B(t, T(t, \ldots, t))$ is a $T$-orbitally lower semicontinuous function, then there exists at least one point $\zeta \in X$ such that $T(\zeta, \ldots, \zeta)=\zeta$.

Proof. For the point $x=\left(u_{1}, \ldots, u_{k}\right)$ from (8) for $M(x):=A(x, T(x))$ and $N(x):=B\left(x_{k}, x_{k+1}\right)$ we obtain that the sequence $\left\{\sup _{i \geq n} M\left(T^{i}(x)\right\}_{n \in \mathbb{N}}\right.$ converges in $\mathbb{R}_{+}^{0}$. Hence, from local sup TCS-convergence, we obtain that $\left\{T^{n}(x)\right\}_{n \in \mathbb{N}}$ has a convergent subsequence $\left\{T^{n(r)}(x)\right\}_{n \in \mathbb{N}}$ with limit $\zeta \in X$. Since $A$ and $B$ satisfy all the required hypotheses in Theorem 3 , we obtain that there exists at least one point $\zeta \in X$ such that $T(\zeta, \ldots, \zeta)=\zeta$. The proof is complete.

Uniqueness of fixed point. Let $X:=(X, A)$ be a topological space and $T: X \rightarrow X$, where $A: X \times X \rightarrow \mathbb{R}_{+}^{0}$. In this part we shall introduce the concept of sup TCS-convergence in a space $X$, i.e., a topological space $X$ satisfies the condition of sup TCS-convergence iff $x \in X$ and $\sup _{i, j \geq n} A\left(T^{i} x, T^{j} x\right)$ or $\sup _{i, j \geq 2 n} A\left(T^{i} x, T^{j} x\right)$ or $\sup _{i, j \geq 2 n+1} A\left(T^{i} x, T^{j} x\right)$ converges to $a, b, c \geq 0$, respectively implies that $\left\{T^{n}(x)\right\}_{n \in \mathbb{N}}$ or $\left\{T^{2 n}(x)\right\}_{n \in \mathbb{N}}$ or $\left\{T^{2 n+1}(x)\right\}_{n \in \mathbb{N}}$ has a convergent subsequence respectively, and if $A(s, t) \leq a, b$ or $c$ implies $s=t$, respectively.

In connection with this for $x, y \in X$ the set $\sigma(x, y, \infty):=\left\{x, y, T x, T y, T^{2} x\right.$, $\left.T^{2} y, \ldots\right\}$ is called the orbit of $x$ and $y$. In this part we begin with a statement which is fundamental for the further considerations. 
Theorem 4. (Corollary of Theorem 3). Let $T$ be a mapping of a topological space $X:=(X, A)$ into itself, where $X$ satisfies the condition of sup TCSconvergence. Suppose that there exists a controlling function $B: X \times X \rightarrow$ $\mathbb{R}_{+}^{0}$ such that

$$
A(T x, T y) \leq B(x, y) \leq \sup _{z, r \in \sigma(x, y, \infty)} A(z, r)<+\infty
$$

for all $x, y \in X$, where $x \mapsto A(x, T x)$ or $x \mapsto B(x, T x)$ is $T$-orbitally lower semicontinuous, then $T$ has at least one fixed point in $X$.

Proof. Let $x \in X$ be an arbitrary point in $X$ and $y=T x$ in (A). Hence we obtain condition (M) in Theorem 3 such that $X$ is in this case with the property of the local sup TCS-convergence. Applying Theorem 3, in this case, we obtain that $T$ has at least one fixed point. The proof is complete.

The following statement give uniqueness of fixed point on topological spaces with the property of TCS-convergence.

Corollary 11. Let $T$ be a mapping of a topological space $X:=(X, A)$ into itself, where $X$ satisfies the condition of TCS-convergence. Suppose that there exists a controlling function $B: X \times X \rightarrow \mathbb{R}_{+}^{0}$ such that

$$
A(T x, T y) \leq B(x, y)<A(x, y) \quad \text { for all } x, y \in X
$$

or

$$
A(T x, T y)<B(x, y) \leq A(x, y) \quad \text { for all } x, y \in X,
$$

where $x \mapsto A(x, T x)$ or $x \mapsto B(x, T x)$ is a $T$-orbitally lower semicontinuous function such that the following inequality holds

$$
\limsup _{n \rightarrow \infty}\left(\sup _{i, j \geq n} B\left(T^{i} x, T^{j} x\right)\right)<\limsup _{n \rightarrow \infty}\left(\sup _{i, j \geq n} A\left(T^{i} x, T^{j} x\right)\right)<+\infty,
$$

then $T$ has a unique fixed point $\zeta \in X$ and $T^{n}(x) \rightarrow \zeta$ as $n \rightarrow \infty$ for arbitrary element $x \in X$.

Proof. This statement is a directly consequence of Theorem 4. Uniqueness follows immediately from condition (9) or $\left(9^{\prime}\right)$. The proof is complete.

Annotation. Let $X:=(X, A)$ be a topological space with the property of sup TCS-convergence. If the conditions (A) and (10) hold, then the space $X$ satisfies also the condition of TCS-convergence.

Theorem 5. (Monotone Principle of F.P.). Let $T$ be a mapping of a topological space $X:=(X, A)$ into itself, where $X$ satisfies the condition of sup TCS-convergence. Suppose that there exists a controlling function $B: X \times X \rightarrow \mathbb{R}_{+}^{0}$ such that

$$
A(T x, T y) \leq B(x, y)<\sup _{z, r \in \sigma(x, y, \infty)} A(z, r)<+\infty
$$


for all $x, y \in X$, or

$$
A(T x, T y)<B(x, y) \leq \sup _{z, r \in \sigma(x, y, \infty)} A(z, r)<+\infty
$$

for all $x, y \in X$, where $x \mapsto A(x, T x)$ or $x \mapsto B(x, T x)$ is T-orbitally lower semicontinuous, then $T$ has at least one fixed point in $X$. If additional $A(t, t) \leq \max \{A(s, t), A(t, s)\}$ for all $s, t \in X$, then $T$ has a unique fixed point $\zeta \in X$ and $T^{n}(x) \rightarrow \zeta$ as $n \rightarrow \infty$ for arbitrary $x \in X$.

Proof. First part of statement is a directly consequence of Theorem 3. Thus the proof of this part we omit. We complete the proof by showing that $T$ can have at most one fixed point: for, if $\zeta \neq \eta$ were two fixed points, then from (11) or (11') we obtain

$$
\begin{aligned}
\max \{A(\zeta, \eta), A(\eta, \zeta)\} & <\max \{A(\zeta, \eta), A(\eta, \zeta), A(\zeta, \zeta), A(\eta, \eta)\}= \\
= & \max \{A(\zeta, \eta), A(\eta, \zeta)\}
\end{aligned}
$$

a contradiction. Thus $T$ has a unique fized point $\zeta \in X$. The proof, together with the first part, is complete.

In connection with the preceding Theorem 5 we obtain, as a main its consequence, Theorem 2 which is a groundwork for further facts in this paper. Indeed, inequality (11) in Theorem 2 has the following equivalent form as a double inequality in the form as

$$
A(T x, T y) \leq \varphi(A(x, y)):=B(x, y)<A(x, y)
$$

for all $x, y \in X$; hence we obtain that (11) holds. Since TCS-convergence implies sup TCS-convergence and since $A$ and $B$ satisfy all required hypotheses in Theorem 5 , hence it follows from Theorem 5 that $T$ has a unique fixed point in $X$.

As an immediate consequence of Theorem 5 we obtain the following global result for sup TCS-convergence on topological spaces.

Corollary 12. Let $T$ be a mapping of a topological space $X:=(X, A)$ into itself, where $X$ satisfies the condition of sup TCS-convergence and for $n \in \mathbb{N} \cup\{0\}$ let

$$
\sup _{i, j \geq n} A\left(T^{i} x, T^{j} x\right)<+\infty \quad \text { for every } x \in X,
$$

where $x \mapsto A(x, T(x))$ is a T-orbitally lower semicontinuous function. Then $T$ has at least one fixed point in $X$. If additional there exists a controlling function $B: X \times X \rightarrow \mathbb{R}_{+}^{0}$ such that (11) or (11'), then $T$ has a unique fixed point $\zeta \in X$ and $T^{n}(x) \rightarrow \zeta$ as $n \rightarrow \infty$ for arbitrary $x \in X$.

Also, an immediate consequence of Theorem 5, in the case of TCSconvergence (i.e., the convergence in zero) we obtain directly the following statement. 
Corollary 13. (Global F.P. Th. for the TCS-convergence). Let $T$ be a mapping of a topological space $X:=(X, A)$ into itself, where $X$ satisfies the condition of TCS-convergence, and for every $x \in X$ the following fact holds

$$
\sup _{i, j \geq n} A\left(T^{i} x, T^{j} x\right) \rightarrow 0 \quad(n \rightarrow \infty),
$$

where $x \mapsto A(x, T x)$ is a T-orbitally lower semicontinuous function, then $T$ has at least one fixed point in $X$. If additional there exists a controlling function $B: X \times X \rightarrow \mathbb{R}_{+}^{0}$ such that (11) or (11'), then $T$ has a unique fixed point $\zeta \in X$ and $T^{n}(x) \rightarrow \zeta$ as $n \rightarrow \infty$ for arbitrary $x \in X$.

Corollary 14. (J. Dugundji and A. Granas [3]). Let $(X, \rho)$ be a complete metric space and $T: X \rightarrow X$ weakly contractive:

$$
\rho[T x, T y] \leq \rho[x, y]-\theta(x, y) \quad \text { for all } x, y \in X,
$$

where $\theta$ is a compactly positive function on $X$. Then $T$ has a unique fixed point $\zeta \in X$ and $T^{n}(x) \rightarrow \zeta$ as $n \rightarrow \infty$ for arbitrary $x \in X$.

Proof. Inequality (DG) has the folowing equivalent form as double inequality with the corresponding controlling function $B$ in the form as

$$
\rho[T x, T y] \leq \rho[x, y]-\theta(x, y):=B(x, y)<\rho[x, y]:=A(x, y)
$$

for all $x, y \in X$. Hence (11) holds. It is easy that $A$ and $B$ satisfy all the required hypotheses in Theorem 5. Since completeness implies sup TCSconvergence, it follows from Theorem 5 that $T$ has a unique fixed point in $X$. The proof is complete.

Corollary 15. (M. A. Krasnoselskij - V. Stečenko [16] and T. Kiventidis [19]). Let $X$ be a Hausdorff space, $T: X \rightarrow X$ a continuous mapping, and $F: X \times X \rightarrow \mathbb{R}_{+}^{0}$ be a continuous function such that $F(x, y) \neq 0$ for all $x, y \in X(x \neq y)$ and

$$
F(T x, T y) \leq F(x, y)-\mu(F(x, y)) \quad \text { for all } x, y \in X,
$$

where $\mu: \mathbb{R}_{+}^{0} \rightarrow \mathbb{R}_{+}^{0}$ is a continuous function with the property $0<\mu(r)<r$ for every $r \in \mathbb{R}_{+}$. If for some $x \in X$ the sequence of iterates $\left\{T^{n}(x)\right\}_{n \in \mathbb{N}}$ has a convergent subsequence, then $T$ has a unique fixed point in $X$.

We notice that in a former paper in 1969 year $\mathrm{Krasnoselskij}$ and $\mathrm{S}$ t e č e n ko [16] investigated a mapping $T$ on a complete metric space $(X, \rho)$ that satisfies condition (KS), i.e., condition (K), for $F(x, y)=\rho[x, y]$, and showed that such mappings have a unique fixed point in $X$. Practically, Corollary 15 is an extension of the result by Krasnoselskij - Stečenko [16] obtained by Kiventidis [19].

Proof of Corollary 15. Let $A(x, y)=F(x, y)$, then $x \mapsto A(x, T x)$ is a $T$-orbitally lower semicontinuous function. Inequality $(\mathrm{K})$ has the following 
equivalent form

$$
F(T x, T y) \leq F(x, y)-\mu(F(x, y)):=B(x, y)<F(x, y):=A(x, y)
$$

for all $x, y \in X$; thus (11) holds. It is easy that $X, A$ and $B$ satisfy all the required hypotheses in Theorem 5 . Applying Theorem 5 we obtain that $T$ has a unique fixed point in $X$. The proof is complete.

Corollary 16. (F. Browder [5], D. W. Boyd - J. S. Wong [8]). Let T be a self-map on a complete metric space $(X, \rho)$. Suppose that there exist an upper semicontinuous from the right function $\Psi$ on $\mathbb{R}_{+}^{0}$ satisfying $\Psi(t)<t$ for every $t>0$ such that

$$
\rho[T x, T y] \leq \Psi(\rho[x, y]) \quad \text { for all } x, y \in X
$$

then $T$ has a unique fixed point $\zeta \in X$ and $\left\{T^{n}(x)\right\}_{n \in \mathbb{N}}$ converges to $\zeta$ for arbitrary element $x \in X$.

Proof. Let $A(x, y):=\rho[x, y]$ and $B(x, y):=\Psi(\rho[x, y])$. It is easy to see that $X, A$ and $B$ satisfy all the required hypotheses in Theorem 5 . This means that $T$ has a unique fixed point in $X$. The proof is complete.

Annotation. We notice that first in 1968 Browder established a fixed point theorem for a self-map $T$ on a complete bounded metric space $(X, \rho)$ satisfying continuous from the right function such that $\Psi(t)<t$ for every $t \in \mathbb{R}_{+}$.

Also, B r ow der in 1968 showed that in the case when $X$ is unbounded, a fixed point theorem if $\Psi: \mathbb{R}_{+}^{0} \rightarrow \mathbb{R}_{+}^{0}$ fulfills the following additional condition in the form: $t-\Psi(t) \rightarrow+\infty$ as $t \rightarrow \infty$. In connection with this the following two conditions are well known:

(12) (A. M e ir and E. K e e le r [26]). For any $\varepsilon>0$ there exists $\delta(\varepsilon)>0$ such that for all $x, y \in X$ the following fact holds in the form as

$$
\rho[T x, T y]<\varepsilon \quad \text { whenever } \quad \varepsilon \leq \rho[x, y]<\varepsilon+\delta(\varepsilon) .
$$

(13) (C. S. Wong [33]). There exists a lower semicontinuous function $f$ : $\mathbb{R}_{+}^{0} \rightarrow \mathbb{R}_{+}^{0}$ satisfying $f(t)>t$ for every $t>0$ such that the following inequality holds in the form as

$$
f(\rho[T x, T y]) \leq \rho[x, y] \text { for all } x, y \in X .
$$

Annotations. We notice that in 1975 year C. S. Wong proved that the following conditions are equivalent: (BW), (12) and (13). For this see Tas k ović [39]. Also, the reference: B r o w d e r [5] contains some applications to differential equations in Banach spaces. Further variants of the Banach fixed point theorem are also contained in: C o l l a t z [27], R u s [20], I s t r ă ţe s c u [28], M i c z k o$\mathrm{P}$ a l c z e w s k i [31], Is é k i [22], and B r ow d e r [7].

In connection with the preceding facts in this context of Theorem 5 the following nonlinear conditions are special cases of the conditions (11) or (11') of Theorem 5:

(14) (J. D a n ěs [24] and M. R. Ta s kovi ć [35]). There exists a nondecreasing function $\Psi: \mathbb{R}_{+}^{0} \rightarrow \mathbb{R}_{+}^{0}$ satisfying: $\Psi^{n}(t) \rightarrow 0(n \rightarrow \infty, t>0)$, and 
$(t-\Psi(t)) \rightarrow+\infty(t \rightarrow \infty)$, such that the following inequality holds in the form

$$
\rho[T x, T y] \leq \Psi(\operatorname{diam}\{x, y, T x, T y\}) \text { for all } x, y \in X .
$$

(15) (Tasković [37]) There exists a nondecreasing function $\Psi: \mathbb{R}_{+}^{0} \rightarrow \mathbb{R}_{+}^{0}$ satisfying $\lim \sup _{z \rightarrow t+0} \Psi(z)<t$ for every $t>0$ such that the following inequality holds in the form:

$$
\rho[T x, T y] \leq \Psi\left(\operatorname{diam}\left\{x, y, T x, T y, \ldots, T^{k} x, T^{m} y\right\}\right)
$$

for arbitrary fixed integers $k, m \geq 0$ and for all points $x, y \in X$. (This is a nonlinear condition for diameter of finite number points.)

(16) (Ta s k o vi ć [39]). Let $T$ be a mapping of a topological space $X:=(X, A)$ into itself and for any $\varepsilon>0$ there exists $\delta=\delta(\varepsilon)>0$ such that for all $x, y \in X$ the following fact holds in the forma as

$$
A(T x, T y)<\varepsilon \quad \text { whenever } \varepsilon \leq \sup _{z, r \in \sigma(x, y, \infty)} A(z, r)<\varepsilon+\delta(\varepsilon) .
$$

(17) (Local form of (16) Ta s k o vi ć [39]). Let $T$ be a mapping of a topological space $X:=(X, M)$ into itself and for $\varepsilon>0$ there exists $\delta=\delta(\varepsilon)>0$ such that for every $x \in X$ the following fact holds in the form as

$$
M(T x)<\varepsilon \quad \text { whenever } \varepsilon \leq \sup _{z \in \sigma(x, \infty)} M(z)<\varepsilon+\delta(\varepsilon) .
$$

Corollary 17. (Tasković [35, p. 250, Theorem 1]). Let $T$ be a mapping of a metric space $(X, \rho)$ into itself and let $X$ be T-orbital complete. Suppose that there exists a function $\varphi: \mathbb{R}_{+}^{0} \rightarrow \mathbb{R}_{+}^{0}$ satisfying

$$
(I \varphi) \quad\left(\forall t \in \mathbb{R}_{+}\right)\left(\varphi(t)<t \quad \text { and } \quad \limsup _{z \rightarrow t+0} \varphi(z)<t\right)
$$

such that the following inequality holds in the form as

$$
\rho[T x, T y] \leq \varphi\left(\operatorname{diam}\left\{x, y, T x, T y, T^{2} x, T^{2} y, \ldots\right\}\right)
$$

for all $x, y \in X$. If $\operatorname{diam}(\sigma(x, \infty)) \in \mathbb{R}_{+}^{0}$ for every $x \in X$, then $T$ has a unique fixed point $\zeta \in X$ and $\left\{T^{n}(a)\right\}_{n \in \mathbb{N}}$ converges to $\zeta$ for arbitrary $a \in X$.

In connection with this Tasković's result we notice that this statement in 1980 year is well-known as: "the finest theorem of nonlinear functional analysis" for metric spaces.

An special case, of the Tasković's condition (J) in 1980 year, appered one year later in Walter's paper as an answer to Browder's result in 1979. But, both conditions (B r ow der [7] and Walter [23]) are very special cases of $(\mathrm{J})$ and $(I \varphi)$ which are give by Tas k ovi ć [35]. We notice that a special case of the conditions $(\mathrm{J})$ and $(I \varphi)$ appeared recently by A k kou chy [58] - 25 years next. But, the author is to neglect and ignore this historical fact! Also, K ir k-S a l i g a [59] are to ignore this historical fact. 
Proof of Corollary 17. Inequality $(\mathrm{J})$ has the following equivalent form as a double inequality with the corresponding controlling function $B$ : $X \times X \rightarrow \mathbb{R}_{+}^{0}$ in the form as

$$
\begin{aligned}
& \rho[T x, T y] \leq \varphi\left(\operatorname{diam}\left\{x, y, T x, T y, T^{2} x, T^{2} y, \ldots\right\}\right):=B(x, y)< \\
& <\operatorname{diam}\left\{x, y, T x, T y, T^{2} x, T^{2} y, \ldots\right\}=\sup _{z, r \in \sigma(x, y, \infty)} A(z, r)<+\infty
\end{aligned}
$$

for all $x, y \in X$, where $A(z, r):=\rho[z, r]$. Hence inequality (11) holds. It is easy that $A$ and $B$ satisfy all the required hypotheses in Theorem 5 . Since $T$-orbitally completeness implies sup TCS-convergence, it follows from Theorem 5 that $T$ has a unique fixed point in $X$. The proof is complete.

Corollary 18. Let $T$ be a mapping of a cartesian product of topological spaces $X^{k}$ ( $k \in \mathbb{N}$ is a fixed number) into $X$, where $X:=(X, A)$ satisfies the condition of sup TCS-convergence. Suppose that there exists a controlling function $B: X \times X \rightarrow \mathbb{R}_{+}^{0}$ such that

$A\left(T\left(u_{1}, \ldots, u_{k}\right), T\left(u_{2}, \ldots, u_{k+1}\right)\right) \leq B\left(u_{k}, u_{k+1}\right)<\sup _{z, r \in \mathfrak{D}(x, T x)} A(z, r)<+\infty$ for all $u_{1}, \ldots, u_{k}, u_{k+1} \in X$ or

$A\left(T\left(u_{1}, \ldots, u_{k}\right), T\left(u_{2}, \ldots, u_{k+1}\right)\right)<B\left(u_{k}, u_{k+1}\right) \leq \sup _{z, r \in \mathfrak{D}(x, T x)} A(z, r)<+\infty$ for all $u_{1}, \ldots, u_{k}, u_{k+1} \in X$, where $x:=\left(u_{1}, \ldots, u_{k}\right)$ and $t \mapsto A(t, T(t, \ldots, t))$ or $t \mapsto B(t, T(t, \ldots, t))$ is a T-orbitally lower semicontinuous function, then there is $\zeta \in X$ such that $T(\zeta, \ldots, \zeta)=\zeta$. If additional $A(t, t) \leq$ $\max \{A(s, t), A(t, s)\}$ for all $s, t \in X$, then there is a unique $\zeta \in X$ such that $T(\zeta, \ldots, \zeta)=\zeta$, and

$$
T^{n}(x):=u_{n+k}=T\left(u_{n}, \ldots, u_{n+k-1}\right) \quad \text { for every } n \in \mathbb{N}
$$

converges to a unique solution $\zeta \in X$ of the equation $T(t, \ldots, t)=t$.

The proof of this statement is a total analogy with the former proof of Corollary 10. Thus the proof we omit.

Transversal upper interval spaces. In connection with the preceding, the function $\rho: X \times X \rightarrow[a, b] \subset \mathbb{R}_{+}^{0}$ for $a<b$ is called an upper (interval) transverse on $X$ (or upper interval transversal) iff: $\rho[x, y]=\rho[y, x]$ and if there is an upper (interval) bisection function $g:[a, b] \times[a, b] \rightarrow[a, b]$ such that

$$
\rho[x, y] \leqslant \max \{\rho[x, z], \rho[z, y], g(\rho[x, z], \rho[z, y])\}
$$

for all $x, y, z \in X$. A transversal upper interval space is a set $X$ together with a given upper intervally transverse $\rho: X \times X \rightarrow[a, b] \subset \mathbb{R}_{+}^{0}$ for $a<b$ on $X$. 
A fundamental first example of upper transversal spaces for the upper bisection function $g:\left(\mathbb{R}_{+}^{0}\right)^{2} \rightarrow \mathbb{R}_{+}^{0}:=[0, \infty)$ defined in the following sense by $g(s, t)=s+t$ is a metric space.

Further, a mapping $M: \mathbb{R} \rightarrow[a, b] \subset \mathbb{R}_{+}^{0}$ for $a<b$ is called an upper (distribution) function if it is nonincreasing, left-continuous with inf $M=a$ and $\sup M=b$. We will denote by $\mathfrak{D}$ the set of all upper (distribution) functions.
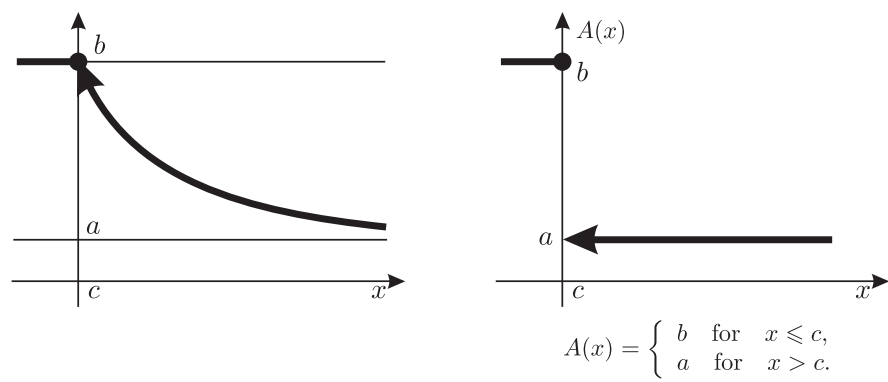

Figure 1

The next two spaces are very interesting examples of transversal upper spaces. First, an upper statistical space is a pair $(X, \mathcal{R})$, where $X$ is an abstract set and $\mathcal{R}$ is a mapping of $X \times X$ into the set of all upper (distribution) functions $\mathfrak{D}$. We shall denote the upper (distribution) function $\mathcal{R}(u, v)$ by $M_{u, v}(x)$ or $M_{u, v}$, whence the symbol $M_{u, v}(x)$ will denote the value of $M_{u, v}$ at $x \in \mathbb{R}$. The functions $M_{u, v}$ are assumed to satisfy the following conditions: $M_{u, v}=M_{v, u}, M_{u, v}(c)=b$ for some $c \in \mathbb{R}$, and

$$
M_{u, v}(x)=a \quad \text { for } \quad x>c \quad \text { if and only if } u=v,
$$

and if $M_{u, r}(x)=a$ and $M_{r, v}(y)=a$ implies $M_{u, v}(x+y)=a$ for all $u, v, r \in X$ and for all $x, y \in \mathbb{R}$.

In view of the condition $M_{u, v}(c)=b$, which evidently, implies that $M_{u, v}(x)=b$ for every $x \leq c$. Thus, condition (Eq) is equivalent to the statement: $u=v$ if and only if $M_{u, v}(x)=A(x)$, where $A(x)=b$ if $x \leq c$ and $A(x)=a$ if $x>c$. See Figure 1.

Obviously, every metric space may be regarded as an upper statistical space of a special kind. One has only to set $M_{u, v}(x)=A(x-d(u, v))$ for every pair of points $(u, v)$ in the metric space $(X, d)$. Also, $M_{u, v}(x)$ may be interpreted as the "measure" that the distance between $u$ and $v$ is less than $x$.

Second example of transversal upper space, an upper interval space (or Taskovićs interval space from Tas k ov ic [46]) is a nonempty set $X$ together with the functions $M_{u, v}(x)$ with the following properties: $M_{u, v}=M_{v, u}, M_{u, v}(c)=b$ for some $c \in \mathbb{R},(\mathrm{Eq})$, and if there is a nondecreasing function $f:[a, b] \times[a, b] \rightarrow[a, b]$ with the property $f(t, t) \leq t$ for all $t \in[a, b]$ such that

$$
M_{u, v}(x+y) \leq f\left(M_{u, r}(x), M_{r, v}(y)\right)
$$


for all $u, v, r \in X$ and for all $x, y \geqslant c$. (Namely, the function $f:[a, b] \times[a, b] \rightarrow[a, b]$ is nondecreasing if $a_{i}, b_{i} \in[a, b]$ and $a_{i} \leq b_{i}(i=1,2)$ implies $f\left(a_{1}, a_{2}\right) \leq$ $f\left(b_{1}, b_{2}\right)$.).

We notice, if we choose an upper bisection (interval) function $g:[a, b] \times[a, b] \rightarrow$ $[a, b]$ such that $g=f$ (from $(\mathrm{Nt})$ ), then we immediately obtain that every upper interval space, for $\rho[u, v]=M_{u, v}$, is a transversal upper interval space; because in this case from $(\mathrm{Nt})$ the following inequalities hold:

$$
\begin{gathered}
\rho[u, v]=M_{u, v}(x) \leq f\left(M_{u, r}(x-y), M_{r, v}(y)\right):= \\
:=g(\rho[u, r], \rho[r, v]) \leqslant \max \{\rho[u, r], \rho[r, v], g(\rho[u, r], \rho[r, v])\} .
\end{gathered}
$$

On the other hand, if: $M_{u, v}=M_{v, u}, M_{u, v}(c)=b$ for some $c \in \mathbb{R}$, (Eq), and if there is a function $\psi:[a, b] \times[a, b] \rightarrow[a, b]$ such that

$$
M_{u, v}(x) \leq \psi\left(M_{u, r}(x), M_{r, v}(x)\right)
$$

for all $u, v, r \in X$ and for every $x \geqslant c$, then it is an example of transversal upper interval space also.

A mapping $\triangle:[0,1] \times[0,1] \rightarrow[0,1]$ is a $\triangle$-norm if it satisfies: $\triangle(a, 1)=$ $a, \triangle(0,0)=0, \triangle(a, b)=\triangle(b, a), \triangle(c, d) \geqslant \triangle(a, b)$ for $c \geqslant a, d \geqslant b$ and $\triangle(\triangle(a, b), c)=\triangle(a, \triangle(b, c))$.

Let $\mathcal{B}$ denote the set of all $\triangle$-norms, partially ordered as by $\triangle_{1} \leqslant \triangle_{2}$ if and only if $\triangle_{1}(a, b) \leqslant \triangle_{2}(a, b)$ for all $a, b \in[0,1]$ and $\triangle_{1}, \triangle_{2} \in \mathcal{B}$.

In connection with this, an upper probabilistic MT-space is a triplet $(X, \mathcal{R}, \triangle)$, where $(X, \mathcal{R})$ is an upper statistical space and $f \in \mathcal{B}$ satisfies the preceding triangle inequality $(\mathrm{Nt})$.

A very characteristic example, for further work, of the transversal upper interval spaces is the following space in the following form.

A transversal upper interval T-space is a pair $(X, \rho)$, where $X$ is a transversal upper interval space and where the upper (interval) transverse $\rho[u, v]=M_{u, v}(x)$ is satisfying: $M_{u, v}=M_{v, u}, M_{u, v}(c)=b$ for some $c \in \mathbb{R}$, and (Eq).

Further, the concept of a neighborhood can be introduced and defined with the aid of the upper interval transverse. In fact, neighborhoods in transversal upper interval spaces may be defined in several nonequivalent ways. Here, we shall consider only one of these.

If $p \in X, \mu>c$ for some $c \in \mathbb{R}$ and $r$ a positive real, then an $(\mu, r)$-neighborhood of $p$, denoted by $U_{p}(\mu, r)$, is defined by

$$
U_{p}(\mu, r)=\left\{q \in X: \rho[p, q]=M_{p, q}(\mu)<a+r\right\} .
$$

Corollary 19. Let $T$ be a mapping of a transversal upper interval $T$-space $X:=\left(X, M_{u, v}(t)\right)$ into itself, where $X$ satisfies the condition of sup TCSconvergence. Suppose that there exists an upper function $K_{x, y}(t)$, as a controlling function, such that

$$
M_{T x, T y}(t) \leq K_{x, y}(\varphi(t))<\sup _{z, r \in \sigma(x, y, \infty)} M_{z, r}(\varphi(t))
$$


for all $x, y \in X$ or

$$
M_{T x, T y}(t)<K_{x, y}(\varphi(t)) \leq \sup _{z, r \in \sigma(x, y, \infty)} M_{z, r}(\varphi(t))
$$

for all $x, y \in X$, where $\varphi: \mathbb{R} \rightarrow \mathbb{R}$ is an increasing function satisfying $\varphi^{n}(t) \rightarrow+\infty(n \rightarrow \infty)$. Then $T$ has a unique fixed point in $X$.

The proof of this statement is a total analogy with the preceding proofs consequences of the main statements. Thus the proof we omit.

We notice that Corollary 19 has an adequate form which is profitable for appliances in the following form.

Corollary 19a.. Let $T$ be a mapping of a transversal upper interval $T$ space $X:=\left(X, M_{u, v}(t)\right)$ into itself, where $X$ satisfies the condition of sup TCS-convergence. Suppose that there exists an upper function $K_{x, y}(t)$, as a controlling function, such that

$$
M_{T x, T y}(\varphi(t)) \leq K_{x, y}(t)<\sup _{z, r \in \sigma(x, y, \infty)} M_{z, r}(\varphi(t))
$$

for all $x, y \in X$ or

$$
M_{T x, T y}(\varphi(t))<K_{x, y}(t) \leq \sup _{z, r \in \sigma(x, y, \infty)} M_{z, r}(\varphi(t))
$$

for all $x, y \in X$, where $\varphi: \mathbb{R} \rightarrow \mathbb{R}$ is an increasing function satisfying $\varphi^{n}(t) \rightarrow+\infty(n \rightarrow \infty)$. Then $T$ has a unique fixed point in $X$.

Expansion Monotone Principles. Let $X:=(X, A)$ be a topological space and $T: X \rightarrow X$, where $A: X \times X \rightarrow \mathbb{R}_{+}^{0}:=[0,+\infty)$. In 1985 year we investigated the concept of CS-convergence in a space $X$. i.e., a topological space $X$ satisfies the condition of CS-convergence iff $\left\{x_{n}\right\}_{n \in \mathbb{N}}$ is a sequence in $X$ and $A\left(x_{n}, T\left(x_{n}\right)\right) \rightarrow 0(n \rightarrow \infty)$ implies that $\left\{x_{n}\right\}_{n \in \mathbb{N}}$ has a convergent subsequence.

Also, we shall introduce the concept of invariant property for space $X$; i.e., a topological space $X$ satisfies the condition of invariant property if there is a nonempty subset $S$ of $X$ such that $T(S)=S$. Obviously, if $T: X \rightarrow X$ is an onto mapping, then $X$ is with the invariant property for $S=X$. Also, if $T: X \rightarrow X$ continuous on a compact space $X$, then $X$ has the invariant property.

We are now in a position to formulate via Axiom of Choice the following general statement of fixed point on topological spaces.

Theorem 6. (General Expansion, Tasković [44]). Let $T$ be a mapping of a topological space $X:=(X, A)$ into itself, where $X$ with the invariant property and with the condition of CS-convergence. If there is a mapping $\varphi: \mathbb{R}_{+}^{0} \rightarrow$ $\mathbb{R}_{+}^{0}$ such that

$$
\left(\forall t \in \mathbb{R}_{+}\right)\left(\varphi(t)>t \quad \text { and } \quad \liminf _{z \rightarrow t-0} \varphi(z)>t\right)
$$


and the following inequality holds in the form as

$$
A(T x, T y) \geq \varphi(A(x, y)) \quad \text { for all } x, y \in X,
$$

where $x \mapsto A(x, T(x))$ is lower semicontinuous and $A(t, s)=0$ implies $t=s$, then $T$ has a unique fixed point in $X$.

In this context, let $X:=(X, M)$ be a topological space and $T: X \rightarrow X$, where $M: X \rightarrow \mathbb{R}_{+}^{0}$. A topological space $X$ satisfies the condition of LCSconvergence iff $\left\{x_{n}\right\}_{n \in \mathbb{N}}$ is a sequence in $X$ and $M\left(x_{n}\right) \rightarrow 0(n \rightarrow \infty)$ implies that $\left\{x_{n}\right\}_{n \in \mathbb{N}}$ has a convergent subsequence.

Theorem 7. (Localization of general expansion, Tasković [44]). Let $T$ be a mapping of a topological space $X:=(X, M)$ into itself, where $X$ with the invariant property and with the condition of LCS-convergence. If there is a mapping $\varphi: \mathbb{R}_{+}^{0} \rightarrow \mathbb{R}_{+}^{0}$ such that the condition $(\varphi r)$ holds and

$$
M(T x) \geq \varphi(M(x)) \quad \text { for every } x \in X,
$$

where $M: X \rightarrow \mathbb{R}_{+}^{0}$ is lower semicontinuous and $M(t)=0$ implies $T(t)=t$, then $T$ has at least one fixed point in $X$.

The proof of this statement is founded and given in 2001 via Axiom of Choice by Tasković [44]. On the other hand, Theorem 6 is an immediate consequence of Theorem 7 .

Let, in the next, $X:=(X, M)$ be a topological space and $T: X \rightarrow X$, where $M: X \rightarrow \mathbb{R}_{+}^{0}$ is a bounded above function. In this part we shall introduce the concept of local inf TCS-convergence in a space $X$, i.e., a topological space $X$ satisfies the condition of local inf TCS-convergence iff $x \in X$ and $\inf _{i \geq n} M\left(T^{i} x\right)$ or $\inf _{i \geq 2 n} M\left(T^{i} x\right)$ or $\inf _{i \geq 2 n+1} M\left(T^{i} x\right)$ converges to $a, b, c \geq 0$ respectively implies that $\left\{T^{n}(x)\right\}_{n \in \mathbb{N}}$ or $\left\{T^{2 n}(x)\right\}_{n \in \mathbb{N}}$ or $\left\{T^{2 n+1}(x)\right\}_{n \in \mathbb{N}}$ has a convergent subsequence respectively, and if $M(t) \geq a$, $b$, or $c$ implies $T(t)=t$, respectively.

Theorem 8. (Localization Monotone Principle). Let $T$ be a mapping of a topological space $X:=(X, M)$ into itself, where $X$ satisfies the condition of local inf TCS-convergence. Suppose that there exists a sontrolling function $N: X \rightarrow \mathbb{R}_{+}^{0}$ such that

$$
M(T x) \geq N(x) \geq \inf _{z \in \sigma(x, \infty)} M(z)>0 \quad \text { for every } x \in X,
$$

where $x \mapsto M(x)$ or $x \mapsto N(x)$ is a T-orbitally upper semicontinuous function. Then $T$ has at least one fixed point in $X$.

The proof of this statement is a total analogy with the former proof of Theorem 3. Thus the proof we omit.

An immediate consequence of the preceding statement is the following result. 
Corollary 20.. Let $T$ be a mapping of a topological space $X:=(X, M)$ into itself, where $X$ satisfies the condition of local inf TCS-convergence. Suppose that there exists a controlling function $N: X \rightarrow \mathbb{R}_{+}^{0}$ such that

$$
M(T x) \geq N(x) \geq M(x) \quad \text { for every } x \in X,
$$

where $M$ or $N$ is $T$-orbitally upper semicontinuous. Then $T$ has at least one fixed point in $X$.

The proof of this statement is an elementary fact because condition (L') implies condition (L). Also, an immediate consequence of Corollary 20 is the following statement.

Corollary 21.. Let $T$ be a mapping of a topological space $X:=(X, M)$ into itself with the property (L'). If for some $x \in X$ the sequence $\left\{T^{n}(x)\right\}_{n \in \mathbb{N}}$ has a convergent subsequence, then $T$ has at least one fixed point in $X$.

In the context of the preceding Theorem 8 we obtain, as a main its consequence, Theorem 7 which is a groundwork for further considerations. Indeed, inequality (LE) in Theorem 7 has the following equivalent form as a double inequality

$$
M(T x) \geq \varphi(M(x)):=N(x)>M(x) \text { for every } x \in X
$$

hence we obtain that (L) holds. Since local TCS-convergence implies local $\inf$ TCS-convergence and since $M$ and $N$ satisfy all the required hypotheses in Theorem 8 , hence it follows from Theorem 8 that $T$ has at least one fixed point in $X$. Thus Theorem 7 is a first directly consequence of Theorem 8 .

Corollary 22.. (Tasković [41]). Let $T$ be a self-map on a complete metric space $(X, d)$. Suppose that there exists an upper semicontinuous bounded above function $G: X \rightarrow \mathbb{R}$ such that

$$
d(x, T(x)) \leq G(T x)-G(x)
$$

for every $x \in X$. Then $T$ has at least one fixed point in $X$.

Proof. Let $M(x)=G(x)$, whish is bounded above and a $T$-orbitally upper semicontinuous function on $X$. Inequality $(\mathrm{R})$ has the following equivalent form

$$
G(T x) \geq G(x)+d(x, T(x)):=N(x) \geq G(x):=M(x)
$$

for every $x \in X$. Hence (L) holds in Theorem 8. Since $X$ satisfies the condition of local inf TCS-convergence ( $X$ is a complete metric space and from $(\mathrm{R})$ for $x_{n}:=T^{n}(x)$ we obtain

$$
\sum_{i=0}^{n} d\left(x_{i}, x_{i+1}\right) \leq G\left(x_{n+1}\right)-G(x),
$$

where $G$ is a bounded above functional, such that $\left\{T^{n} x\right\}_{n \in \mathbb{N}}$ converges to some $\zeta \in X$ ), applying Theorem $8, T$ has at least one fixed point in $X$. The proof is complete. 
Corollary 23.. (Tasković [43]). Let $T$ be a self-map on a complete metric space $(X, \rho)$. Suppose that there exists an upper semicontinuous bounded above function $G: X \rightarrow[a,+\infty)$ for some $a>0$ such that

$$
\rho[x, T(x)] \leq \frac{1}{G(x)}-\frac{1}{G(T x)} \quad \text { for every } x \in X,
$$

then $T$ has at least one fixed point in $X$.

Proof. Inequality (15) has the following equivalent form as a double inequality with the corresponding controlling function $N$ in the form as

$$
G(T x) \geq \frac{1}{\frac{1}{G(x)}-\rho[x, T(x)]}:=N(x) \geq G(x):=M(x)
$$

for every $x \in X$; hence (L) holds. Since completeness implies local inf TCSconvergence for $M(x)=G(x)$, applying Theorem 8 it follows that $T$ has at least one fixed point in $X$. The proof is complete.

Corollary 24.. Let $T$ be a mapping of a topological space $X:=(X, M)$ into itself, where $X$ satisfies the condition of local inf TCS-convergence, and for $n \in \mathbb{N} \cup\{0\}$ let

$$
\inf _{i \geq n} M\left(T^{i}(x)\right)>0 \quad \text { for every } x \in X,
$$

where $x \mapsto M(x)$ is a T-orbitally upper semicontinuous function. Then $T$ has at least one fixed point in $X$.

The proof of this statement is a total analogous with the former proof of Corollary 9. Thus proof we omit.

Uniqueness of fixed point. Let $X:=(X, A)$ be a topological space and $T: X \rightarrow X$, where $A: X \times X \rightarrow \mathbb{R}_{+}^{0}$. In this part we shall introduce the concept of inf TCS-convergence in a space $X$, i.e., a topological space $X$ satisfies the condition of inf TCS-convergence iff $x \in X$ and $\inf _{i, j \geq n} A\left(T^{i} x, T^{j} x\right)$ or $\inf _{i, j \geq 2 n} A\left(T^{i} x, T^{j} x\right)$ or $\inf _{i, j \geq 2 n+1} A\left(T^{i} x, T^{j} x\right)$ converges to $a, b, c \geq 0$ respectively implies that $\left\{T^{n}(x)\right\}_{n \in \mathbb{N}}$ or $\left\{T^{2 n}(x)\right\}_{n \in \mathbb{N}}$ or $\left\{T^{2 n+1}(x)\right\}_{n \in \mathbb{N}}$ has a convergent subsequence respectively, and if $A(s, t) \geq a, b$ or $c$ implies $s=t$, respectively.

Theorem 9. (Monotony General Expansion, Tasković [51]). Let $T$ be a mapping of a topological space $X:=(X, A)$ into itself, where $X$ satisfies the condition of inf TCS-convergence. Suppose that there exists a controlling function $B: X \times X \rightarrow \mathbb{R}_{+}^{0}$ such that

$$
A(T x, T y) \geq B(x, y)>\inf _{z, r \in \sigma(x, y, \infty)} A(z, r)>0
$$

for all $x, y \in X$, or

$$
A(T x, T y)>B(x, y) \geq \inf _{z, r \in \sigma(x, y, \infty)} A(z, r)>0
$$


for all $x, y \in X$, where $x \mapsto A(x, T x)$ or $x \mapsto B(x, T x)$ is T-orbitally upper semicontinuous, then $T$ has at lest one fixed point in $X$. If $A(t, t) \geq$ $\min \{A(t, s), A(s, t)\}$ for all $s, t \in X$, then $T$ has a unique fixed point $\zeta \in X$ and $T^{n}(x) \rightarrow \zeta$ as $n \rightarrow \infty$ for arbitrary $x \in X$.

The proof of this statement is a total analogy with the former proof of Theorem 5. Thus the proof we omit.

As an immediate consequence of Theorem 9 directly we obtain the following statement on topological spaces.

Corollary 25.. (Global General Expansion). Let $T$ be a mapping of a topological space $X:=(X, A)$ into itself, where $X$ satisfies the condition of inf TCS-convergence and for $n \in \mathbb{N} \cup\{0\}$ let

$$
\inf _{i, j \geq n} A\left(T^{i} x, T^{j} x\right)>0 \quad \text { for every } x \in X,
$$

where $x \mapsto A(x, T(x))$ is a T-orbitally upper semicontinuous function. Then $T$ has at least one fixed point in $X$. In additional, if there exists a controlling function $B: X \times X \rightarrow \mathbb{R}_{+}^{0}$ such that

$$
A(T x, T y) \geq B(x, y)>A(x, y) \quad \text { for all } x, y \in X
$$

or

$$
A(T x, T y)>B(x, y) \geq A(x, y) \quad \text { for all } x, y \in X,
$$

then $T$ has a unique fixed point $\zeta \in X$ and $T^{n}(x) \rightarrow \zeta$ as $n \rightarrow \infty$ for arbitrary $x \in X$.

In [60] Wang, Gao, Li and Iséki proved the following statement for a class of expansive mappings. Namely, if $(X, d)$ is a complete metric space, if a mapping $T: X \rightarrow X$ is onto and if there exists $q>1$ such that

$$
d(T(x), T(y)) \geq q d(x, y) \text { for all } x, y \in X,
$$

then $T$ has a unique fixed point in $X$. Also, Daffer and Kaneko [61] proved: If $T$ is a continuous compact mapping of a metric space $(X, d)$ into itself satisfying the expansive condition (Ik), then $T$ has a unique fixed point in $X$.

We notice that the preceding two results are immediate consequences of Theorem 9. Also see: Tasković [37, p. 62].

Transversal lower interval spaces. In connection with the preceding, the function $\rho: X \times X \rightarrow[a, b] \subset \mathbb{R}_{+}^{0}$ for $a<b$ is called a lower (interval) transverse on $X$ (or lower interval transversal) iff: $\rho[x, y]=\rho[y, x]$ and if there is a lower (interval) bisection function $d:[a, b] \times[a, b] \rightarrow[a, b]$ such that

$$
\rho[x, y] \geqslant \min \{\rho[x, z], \rho[z, y], d(\rho[x, z], \rho[z, y])\}
$$


for all $x, y, z \in X$. A transversal lower interval space is a set $X$ together with a given lower interval transverse $\rho: X \times X \rightarrow[a, b] \subset \mathbb{R}_{+}^{0}$ for $a<b$ on $X$.

Otherwise, a transversal interval space (or a middle transversal interval space) is an upper and a lower transversal interval space simultaneously.

As an important example of transversal lower interval spaces we have a Menger's (probabilistic) space. K a r l M e n g e r introduced in 1942 the notion of probabilistic metric space.

In this sense, a mapping $N: \mathbb{R} \rightarrow[a, b] \subset \mathbb{R}_{+}^{0}$ for $a<b$ is called a lower (distribution) function if it is nondecreasing, left-continuous with inf $N=$ $a$ and $\sup N=b$. We will denote by $\mathcal{L}$ the set of all lower (distribution) functions.

A lower statistical space is a pair $(X, \mathscr{D})$, where $X$ is an abstract set and $\mathscr{D}$ is a mapping of $X \times X$ into the set of all lower (distribution) functions $\mathcal{L}$. We shall denote the lower (distribution) function $\mathscr{D}(p, q)$ by $N_{p, q}(x)$ or $N_{p, q}$, whence the symbol $N_{p, q}(x)$ will denote the value of $N_{p, q}$ at $x \in \mathbb{R}$. The functions $N_{p, q}$ are assumed to satisfy the following conditions: $N_{p, q}=N_{q, p}, N_{p, q}(c)=a$ for some $c \in \mathbb{R}$, and

$$
N_{p, q}(x)=b \quad \text { for } \quad x>c \quad \text { if and only if } p=q,
$$

and if $N_{p, q}(x)=b$ and $N_{q, r}(y)=b$ implies $N_{p, r}(x+y)=b$ for all $p, q, r \in X$ and for all $x, y \in \mathbb{R}$.

In view of the condition $N_{p, q}(c)=a$ for some $c \in \mathbb{R}$, which evidently, implies that $N_{p, q}(x)=a$ for all $x \leq c$. Thus, the condition (Em) is equivalent to the statement: $p=q$ if and only if $N_{p, q}(x)=H(x)$, where $H(x)=a$ if $x \leq c$ and $H(x)=b$ if $x>c$. See Figure 2 .
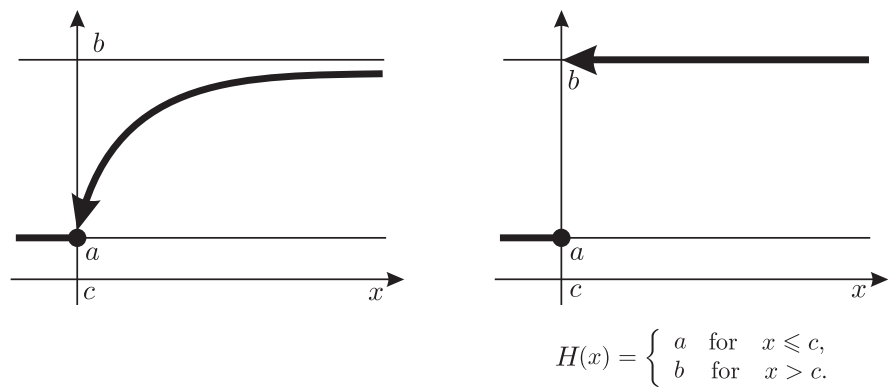

FiguRE 2

Every metric space may be regarded as a statistical lower space of a special kind. One has only to set $N_{p, q}(x)=H(x-\rho(p, q))$ for every pair of points $(p, q)$ in the metric space $(X, \rho)$.

An example of transversal lower interval space is a lower interval space which is a nonempty set $X$ together with the functions $N_{p, q}(x)$ with the following properties: $N_{p, q}=N_{q, p}, N_{p, q}(c)=a$ for some $c \in \mathbb{R},($ Em), and if there is a nondecreasing 
function $\tau:[a, b] \times[a, b] \rightarrow[a, b]$ with the property $\tau(t, t) \geqslant t$ for all $t \in[a, b]$ such that

$(\mathrm{Nm})$

$$
N_{p, q}(x+y) \geqslant \tau\left(N_{p, r}(x), N_{r, q}(y)\right)
$$

for all $p, q, r \in X$ and for all $x, y \geqslant c$.

We notice, if we choose a lower (interval) bisection function $d:[a, b] \times[a, b] \rightarrow$ $[a, b]$ such that $d=\tau$ (from $(\mathrm{Nm})$ ), then we immediately obtain that every lower interval space, for $\rho[p, q]=N_{p, q}$, is a transversal lower interval space; because in this case from $(\mathrm{Nm})$ the following inequalities hold:

$$
\begin{gathered}
\rho[p, q]=N_{p, q}(x) \geqslant \tau\left(N_{p, r}(x-y), N_{r, q}(y)\right):= \\
:=d(\rho[p, r], \rho[r, q]) \geqslant \min \{\rho[p, r], \rho[r, q], d(\rho[p, r], \rho[r, q])\} .
\end{gathered}
$$

In connection with the preceding, a transversal lower interval T-space is a pair $(X, \rho)$, where $X$ is a transversal lower interval space and where the lower (interval) transverse $\rho[u, v]=N_{u, v}(x)$ is satisfying: $N_{u, v}=N_{v, u}, N_{u, v}(c)=a$ for some $c \in \mathbb{R}$ and (Em). This space is a very characteristic example of transversal lower interval spaces for further work.

A Menger space (or lower probabilistic MT-space) is a triplet $(X, \mathscr{D}, \triangle)$, where $(X, \mathscr{D})$ is a lower statistical space, where $\rho[u, v]=F_{u, v}(x): X \times X \rightarrow[0,1]$ and $\tau \in \mathcal{B}$ satisfies the preceding triangle inequality $(\mathrm{Nm})$.

If we choose a lower bisection function $d:[0,1] \times[0,1] \rightarrow[0,1]$ such that $d=\tau$ (for $\tau \in \mathcal{B}$ ) then from $(\mathrm{N} \tau)$ we immediately obtain that every Menger's space, for $\rho[p, q]=F_{p, q}$, is a transversal lower interval space. Every Menger's space is a lower interval space also.

The concept of a neighborhood in a lower transversal interval space $X$ for the lower interval transverse $\rho[p, q]=N_{p, q}(x)$ in $[a, b] \subset \mathbb{R}_{+}^{0}$ for $a<b$ is the following. If $p \in X, \mu>c$ for some $c \in \mathbb{R}$, and $\sigma$ a positive real, then an $(\mu, \sigma)$-neighborhood of $p$ denoted by $\mathcal{O}_{p}(\mu, \sigma)$, is defined by

$$
\mathcal{O}_{p}(\mu, \sigma)=\left\{q \in X: \rho[p, q]=N_{p, q}(\mu)>b-\sigma\right\}
$$

Corollary 26.. Let $T$ be a mapping of a transversal lower interval $T$-space $X:=\left(X, N_{u, v}(t)\right)$ into itself, where $X$ satisfies the condition of inf TCSconvergence. Suppose that there exists a lower function $K_{x, y}(t)$, as a controlling function, such that

$$
N_{T x, T y}(t) \geq K_{x, y}(\varphi(t))>\inf _{z, r \in \sigma(x, y, \infty)} N_{z, r}(\varphi(t))
$$

for all $x, y \in X$ or

$$
N_{T x, T y}(t)>K_{x, y}(\varphi(t)) \geq \inf _{z, r \in \sigma(x, y, \infty)} N_{z, r}(\varphi(t))
$$

for all $x, y \in X$, where $\varphi: \mathbb{R} \rightarrow \mathbb{R}$ is an increasing function satisfying $\varphi^{n}(t) \rightarrow+\infty(n \rightarrow \infty)$. Then $T$ has a unique fixed point in $X$.

The proof of this statement is a total analogy with the preceding proofs consequences of the main statements. Thus the proof we omit. 
We notice that Corollary 26 has an adequate form which is profitable for appliances in the following form.

Corollary 26a.. Let $T$ be a mapping of a transversal lower interval $T$ space $X:=\left(X, N_{u, v}(t)\right)$ into itself, where $X$ satisfies the condition of inf TCS-convergence. Suppose that there exists a lower function $K_{x, y}(t)$, as a controlling function, such that

$$
N_{T x, T y}(\varphi(t)) \geq K_{x, y}(t)>\inf _{z, r \in \sigma(x, y, \infty)} N_{z, r}(\varphi(t))
$$

for all $x, y \in X$ or

$$
N_{T x, T y}(\varphi(t))>K_{x, y}(t) \geq \inf _{z, r \in \sigma(x, y, \infty)} N_{z, r}(\varphi(t))
$$

for all $x, y \in X$, where $\varphi: \mathbb{R} \rightarrow \mathbb{R}$ is an increasing function satisfying $\varphi^{n}(t) \rightarrow+\infty(n \rightarrow \infty)$. Then $T$ has a unique fixed point in $X$.

\section{Monotony and Fixed Apices}

A map $f$ of a partially ordered set $P$ to itself has a fixed point, if there exists an element $\xi$ in $P$ such that $f(\xi)=\xi$, i.e., if the mapping $f$ "is crossing of diagonal" in the fixed point $\xi \in P$ as on Fig. 3
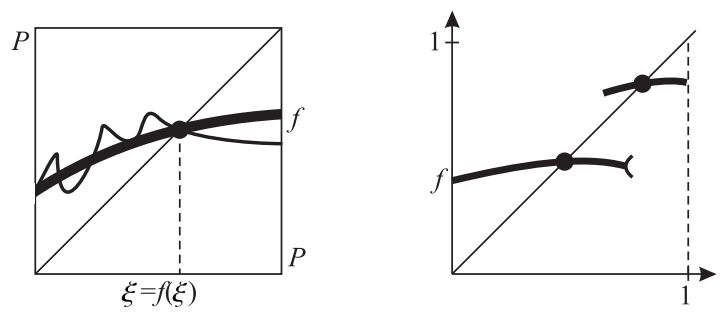

Figure 3

In a paper of mine in 1988 I investigated the concept of fixed apices for a mapping $f$ of a poset $P$ into itself. A map $f$ of a set $P$ to itself has a fixed apex $u \in P$ iff for $u \in P$ there is $v \in P$ such that $f(u)=v$ and $f(v)=u$. The points $u, v \in P$ are called fixed apices of $f$ if $f(u)=v$ and $f(v)=u$, as on Fig. 4.

We notice that fixed points are evidently fixed apices and the set of all fixed points can be a proper subset of the set of fixed apices. Also, a fixed point is evidently a fixed apex.

On the other hand, we notice that the map $f$ has a fixed apex if and only if $f^{2}:=f(f)$ has a fixed point.

Namely, if $f$ has a fixed apex $u \in P$, then $u=f(v)$ and $v=f(u)$, so $f^{2}$ has a fixed point. Reversed, if the equation $x=f^{2}(x)$ has a solution $\xi=f^{2}(\xi)$ for some $\xi \in P$, then $f$ has fixed apices $\xi, f(\xi) \in P$, because $\xi=f^{2}(\xi)$ and $f(\xi)=f(\xi)$. 

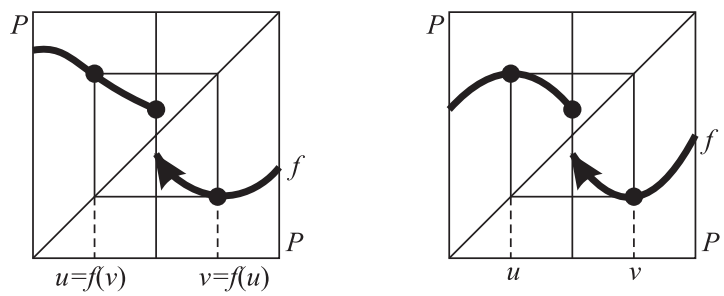

FIGURE 4

In connection with this, we can extend the term apex with $f^{2}$ on the map $f^{n}:=f\left(f^{n-1}\right)$ for an arbitrary fixed integer $n \geqslant 2$. In this sense, some points $u_{1}, \ldots, u_{n} \in P$ are fixed apices for the map $f: P \rightarrow P$ iff

$$
u_{1}=f\left(u_{2}\right), \ldots, u_{n-1}=f\left(u_{n}\right) \text { and } u_{n}=f\left(u_{1}\right),
$$

or, in the reversed direction, iff

$$
u_{2}=f\left(u_{1}\right), \ldots, u_{n}=f\left(u_{n-1}\right) \quad \text { and } \quad u_{1}=f\left(u_{n}\right),
$$

for an arbitrary fixed integer $n \geqslant 2$.

As for case $n=2$, in the place, the map $f$ has fixed apices $u_{1}, \ldots, u_{n} \in P$ if and only if the iteration $f^{n}$, for an arbitrary fixed integer $n \geqslant 2$, has a fixed point. Precisely, we have the following fact.

Proposition 1. Let $X$ be an arbitrary nonempty set, let $T$ be a mapping from $X$ into $X$, and let $n \geqslant 2$ be an arbitrary fixed integer. Then, $T$ has fixed apices $u_{1}, \ldots, u_{n} \in X(n \geqslant 2)$ if and only if the iteration mapping $T^{n}$ $(n \geqslant 2)$ has at least one fixed point.

A brief proof for $n=2$ of this statement (in the case of partially ordered sets) may be found in Tasković [45].

Let $X:=(X, M)$ be a topological space and $T: X \rightarrow X$, where $M$ : $X \rightarrow \mathbb{R}_{+}^{0}$. In this part we shall introduce the concept of local $k$-sup TCSconvergence in a space $X$, i.e., a topological space $X$ satisfies the condition of local $k$-sup TCS-convergence iff $x \in X, k \in \mathbb{N}$ is a fixed number, and $\sup _{i \geq n} M\left(T^{i} x\right)$ or $\sup _{i \geq 1+n k} M\left(T^{i} x\right)$ or, $\ldots$, or $\sup _{i \geq k+n k} M\left(T^{i} x\right)$ converges to $\alpha_{0}, \alpha_{1}, \ldots, \alpha_{k} \geq 0$ respectively implies that $\left\{T^{n}(x)\right\}_{n \in \mathbb{N}}$ or $\left\{T^{n k+1}(x)\right\}_{n \in \mathbb{N}}$ or, $\ldots$, or $\left\{T^{n k+k}(x)\right\}_{n \in \mathbb{N}}$ has a convergent subsequence respectively, and if $M\left(T^{k}(t)\right) \leq \alpha_{i}(i=0,1, \ldots, k)$ implies $T^{k}(t)=t$, respectively.

We are now in a position to formulate the following our theorem on topological spaces for the fixed apices.

Theorem 10. (Localization Monotone Principle for F. A.). Let $T$ be a mapping of a topological space $X:=(X, M)$ into itself, where $X$ satisfies the condition of local $k$-sup TCS-convergence. Suppose that there exists a 
controlling function $N: X \rightarrow \mathbb{R}_{+}^{0}$ such that

(Ma) $\quad M\left(T^{k}(x)\right) \leq N(x) \leq \sup _{z \in \sigma(x, \infty)} M(z)<+\infty \quad$ for every $x \in X$,

where $x \mapsto M\left(T^{k}(x)\right)$ or $x \mapsto N\left(T^{k}(x)\right)$ is a $T$-orbitally lower semicontinuous function. Then $T$ has at least one fixed $k$-apex in $X$.

As an immediate consequence of this statement we have the following result for fixed apices on topological spaces.

Corollary 27.. Let $T$ be a mapping of a topological space $X:=(X, M)$ into itself, where $X$ satisfies local $k$-sup TCS-convergence. Suppose that there exists a controlling function $N: X \rightarrow \mathbb{R}_{+}^{0}$ such that

$$
M\left(T^{k}(x)\right) \leq N(x) \leq M(x) \text { for every } x \in X,
$$

where $x \mapsto M\left(T^{k}(x)\right)$ or $x \mapsto N\left(T^{k}(x)\right)$ is a T-orbitally lower semicontinuous function. Then $T$ has at least one fixed $k$-apex in $X$.

Proof of Theorem 10. Let $x \in X$ be an arbitrary point. From (Ma) for $T^{i} x$ we obtain $M\left(T^{i+k}(x)\right) \leq N\left(T^{i}(x)\right) \leq \sup _{z \in \sigma\left(T^{i} x, \infty\right)} M(z)$, and hence

$$
\sup _{i \geq n+k} M\left(T^{i}(x)\right) \leq \sup _{i \geq n} N\left(T^{i} x\right) \leq \sup _{i \geq n} M\left(T^{i} x\right),
$$

i.e., we obtain that the corresponding sequences of the forms $\left.\sup _{i \geq n} M\left(T^{i} x\right)\right\}_{n \in \mathbb{N}}$, $\left\{\sup _{i \geq 1+n k} M\left(T^{i} x\right)\right\}_{n \in \mathbb{N}}, \ldots,\left\{\sup _{i \geq k+n k} M\left(T^{i} x\right)\right\}_{n \in \mathbb{N}}$ converges decreasing in $\mathbb{R}_{+}^{0}$. This implies (from local $k$-sup TCS-convergence) that their sequences of iterates contains convergent subsequences $\left\{T^{i+n(r) k}(x)\right\}_{r \in \mathbb{N}}$ with limit points $\zeta_{i} \in X(i=0,1, \ldots, k)$. Since $x \mapsto M\left(T^{k}(x)\right)$ is $T$-orbitally lower semicontinuous,

$$
\begin{aligned}
M\left(T^{k}\left(\zeta_{i}\right)\right) & \leq \liminf _{r \rightarrow \infty} M\left(T^{k}\left(T^{i+n(r) k}(x)\right)=\liminf _{r \rightarrow \infty} M\left(T^{i+(n(r)+1) k}(x)\right)=\right. \\
& =\liminf _{n \rightarrow \infty} M\left(T^{i+(n+1) k}(x)\right)=\alpha_{i} \quad(i=0,1, \ldots, k)
\end{aligned}
$$

respectively, which means that $T^{k}\left(\zeta_{i}\right)=\zeta_{i}(i=0,1, \ldots, k)$. This means from Proposition 1 that $T$ has at least $k+1$ points of $k$-fixed apices. If the controlling function $x \mapsto N\left(T^{k}(x)\right)$ is a $T$-orbitally lower semicontinuous function, then we have

$$
\begin{gathered}
N\left(T^{k}\left(\zeta_{i}\right)\right) \leq \liminf _{r \rightarrow \infty} N\left(T^{i+(n(r)+1) k}(x)\right) \leq \\
\leq \liminf _{n \rightarrow \infty}\left(\sup _{j \geq n} N\left(T^{i+j}(x)\right) \leq\right. \\
\leq \liminf _{n \rightarrow \infty}\left(\sup _{j \geq n} M\left(T^{i+j}(x)\right)=\alpha_{i} \quad(i=0,1, \ldots, k),\right.
\end{gathered}
$$

which means that $T^{k}\left(\zeta_{i}\right)=\zeta_{i}(i=0,1, \ldots, k)$, also, i.e., $T$ has (from Proposition 1$)$ at least $k+1$ points of $k$-fixed apices. The proof is complete. 
Corollary 28.. (Local Form of $k$-Global F. A. Th.). Let $T$ be a mapping of a topological space $X:=(X, M)$ into itself, where $X$ satisfies the condition of local $k$-sup TCS-convergence, and for $n \in \mathbb{N} \cup\{0\}$ let

$$
\sup _{i \geq n} M\left(T^{i}(x)\right)<+\infty \quad \text { for every } x \in X,
$$

where $x \mapsto M\left(T^{k}(x)\right)$ is a T-orbitally lower semicontinuous function, then $T$ has at least one fixed $k$-apex in $X$.

The proof of this statement is a total analogy with the former proof of Corollary 9. Thus the proof we omit.

Let $X:=(X, A)$ be a topological space and $T: X \rightarrow X$, where $A$ : $X \times X \rightarrow \mathbb{R}_{+}^{0}$. In this part we shall introduce the concept of $k$-sup TCSconvergence in a space $X$, i.e., a topological space $X$ satisfies the condition of $k$-sup TCS-convergence iff $x \in X, k \in \mathbb{N}$ is a fixed number, and $\sup _{i, j \geq n} A\left(T^{i} x, T^{j} x\right)$ or $\sup _{i, j \geq 1+n k} A\left(T^{i} x, T^{j} x\right)$ or, $\ldots$, or $\sup _{i, j \geq k+n k} A\left(T^{i} x, T^{j} x\right)$ converges to $\alpha_{0}, \alpha_{1}, \ldots, \alpha_{k} \geq 0$ respectively implies that $\left\{T^{n}(x)\right\}_{n \in \mathbb{N}}$ or $\left\{T^{n k+1}(x)\right\}_{n \in \mathbb{N}}$ or, $\ldots$, or $\left\{T^{n k+k}(x)\right\}_{n \in \mathbb{N}}$ has a convergent subsequence respectively, and if $A\left(x, T^{k}(x)\right) \leq \alpha_{i}(i=0,1, \ldots, k)$ implies $T^{k}(t)=t$, respectively.

As an immediate application of Theorem 10 we obtain the following form of the monotone principle for fixed apices on topological spaces.

Theorem 11. (Monotone Principle of F. A.). Let $T$ be a mapping of a topological space $X:=(X, A)$ into itself, where $X$ satisfies the condition of $k$-sup TCS-convergence. Suppose that there exists a controlling function $B: X \times X \rightarrow \mathbb{R}_{+}^{0}$ such that

$$
A\left(T^{k} x, T^{k} y\right) \leq B(x, y) \leq \sup _{z, r \in \sigma(x, y, \infty)} A(z, r)<+\infty
$$

for all $x, y \in X$, where $x \mapsto A\left(x, T^{k} x\right)$ or $x \mapsto B\left(x, T^{k} x\right)$ is T-orbitally lower semicontinuous, then $T$ has at least one $k$-fixed apex in $X$.

As an immediate consequence of this statement we obtain the following result for uniqueness fixed apices on topological spaces.

Corollary 29.. Let $T$ be a mapping of a topological space $X:=(X, A)$ into itself, where $X$ satisfies $k$-sup TCS-convergence. Suppose that there exists a controlling function $B: X \times X \rightarrow \mathbb{R}_{+}^{0}$ such that

$$
A\left(T^{k}(x), T^{k}(y) \leq B(x, y)<A(x, y)\right.
$$

for all $x, y \in X$, or

$$
A\left(T^{k}(x), T^{k}(y)\right)<B(x, y) \leq A(x, y)
$$

for all $x, y \in X$, where $x \mapsto A\left(x, T^{k} x\right)$ or $x \mapsto B\left(x, T^{k} x\right)$ is a T-orbitally lower semicontinuous function. Then $T$ has a unique $k$-fixed apex in $X$. 
We complete the proof by showing that $T$ can have at most one $k$-fixed apex: for, if $\zeta \neq \eta$ were two $k$-fixed apices, then from (23) and (23') we obtain

and

$$
A(\zeta, \eta)=A\left(T^{k}(\zeta), T^{k}(\eta)\right) \leq B(\zeta, \eta)<A(\zeta, \eta)
$$

$$
A(\zeta, \eta)=A\left(T^{k}(\zeta), T^{k}(\eta)\right)<B(\zeta, \eta) \leq A(\zeta, \eta),
$$

two contradictions. The proof of this fact is complete. The proof of the first part of statement is a total analogy with the former proofs. Thus further proof we omit.

As an immediate consequence of Theorem 11 we obtain the following global result for $k$-sup TCS-convergence on topological spaces.

Corollary 30.. Let $T$ be a mapping of a topological space $X:=(X, A)$ into itself, where $X$ satisfies the condition of $k$-sup TCS-convergence and for $n \in \mathbb{N} \cup\{0\}$ let

$$
\sup _{i, j \geq n} A\left(T^{i} x, T^{j} x\right)<+\infty \quad \text { for every } x \in X,
$$

where $x \mapsto A\left(x, T^{k} x\right)$ is a T-orbitally lower semicontinuous function. Then $T$ has at least one $k$-fixed apex in $X$.

If additional there exists a controlling function $B: X \times X \rightarrow \mathbb{R}_{+}^{0}$ such that (23) or (23'), then $T$ has a unique $k$-fixed apex in $X$.

Also, as an immediate consequence of Theorem 10, in the case of TCSconvergence (i.e, the convergence in zero) we obtain directly the following result.

Corollary 31.. Let $T$ be a mapping of a topological space $X:=(X, A)$ into itself, where $X$ satisfies the condition of TCS-convergence and for $x \in X$ the following fact holds

$$
\sup _{i, j \geq n} A\left(T^{i} x, T^{j} x\right) \rightarrow 0 \quad(n \rightarrow \infty),
$$

where $x \mapsto A\left(x, T^{k}(x)\right)$ is a T-orbitally lower semicontinuous function, then $T$ has at least one $k$-fixed apex in $X$.

If additional there exist a controlling function $B: X \times X \rightarrow \mathbb{R}_{+}^{0}$ such that (23) or (23'), then $T$ has a unique $k$-fixed apex in $X$.

Let $X:=(X, M)$ be a topological space and $T: X \rightarrow X$, where $M$ : $X \rightarrow \mathbb{R}_{+}^{0}$. In this part we shall introduce the concept of local $k$-inf TCS-convergence in a space $X$, i.e, a topological space $X$ satisfies the condition of local $k$-inf TCS-convergence iff $x \in X, k \in \mathbb{N}$ is a fixed number, and $\inf _{i \geq n} M\left(T^{i} x\right)$ or $\inf _{i \geq 1+n k} M\left(T^{i} x\right)$ or, $\ldots$, or $\inf _{i \geq k+n k} M\left(T^{i} x\right)$ converges to $\alpha_{0}, \alpha_{1}, \ldots, \alpha_{k} \geq 0$ respectively implies that $\left\{T^{n}(x)\right\}_{n \in \mathbb{N}}$ or $\left\{T^{n k+1}(x)\right\}_{n \in \mathbb{N}}$ or, ..., or $\left\{T^{n k+k}(x)\right\}_{n \in \mathbb{N}}$ has a convergent subsequence respectively, and if $M\left(T^{k}(t)\right) \geq \alpha_{i}(i=0,1, \ldots, k)$ implies $T^{k}(t)=t$, respectively. 
We are now in a position to formulate the following our theorem on topological spaces for fixed apices.

Theorem 12. (Localization of General Expansion for F. A.). Let $T$ be a mapping of a topological space $X:=(X, M)$ into itself, where $X$ satisfies the condition of local $k$-inf TCS-convergence. Suppose that there exists a controlling function $N: X \rightarrow \mathbb{R}_{+}^{0}$ such that

$$
M\left(T^{k}(x)\right) \geq N(x) \geq \inf _{z \in \sigma(x, \infty)} M(z)>0 \quad \text { for every } x \in X,
$$

where $x \mapsto M\left(T^{k}(x)\right)$ or $x \mapsto N\left(T^{k}(x)\right)$ is a $T$-orbitally upper semicontinuous function. Then $T$ has at least one $k$-fixed apex in $X$.

As an immediate consequence of this statement we have the following result for fixed apices on topological space.

Corollary 32.. Let $T$ be a mapping of a topological space $X:=(X, M)$ into itself, where $X$ satisfies the condition of local $k$-inf TCS-convergence. Suppose that there exists a controlling function $N: X \rightarrow \mathbb{R}_{+}^{0}$ such that

$$
M\left(T^{k}(x)\right) \geq N(x) \geq M(x) \quad \text { for every } x \in X,
$$

where $x \mapsto M\left(T^{k} x\right)$ or $x \mapsto N\left(T^{k} x\right)$ is a T-orbitally upper semicontinuous function. Then $T$ has at leat one $k$-fixed apex in $X$.

As an immediate consequence of the preceding two results directly we obtain a global statement on topological spaces.

Corollary 33.. Let $T$ be a mapping of a topological space $X:=(X, M)$ into itself, where $X$ satisfies the condition of local $k$-inf TCS-convergence, and for $n \in \mathbb{N} \cup\{0\}$ let

$$
\inf _{i \geq n} M\left(T^{i} x\right)>0 \quad \text { for every } x \in X,
$$

where $x \mapsto M\left(T^{k}(x)\right)$ is a T-orbitally upper semicontinuous function. Then $T$ has at least one $k$-fixed apex in $X$.

Let $X:=(X, A)$ be a topological space and $T: X \rightarrow X$, where $A$ : $X \times X \rightarrow \mathbb{R}_{+}^{0}$. In this part we shall introduce the concept of $k$-inf TCSconvergence in a space $X$, i.e., a topological space $X$ satisfies the condition of $k$-inf TCS-convergence iff $x \in X, k \in \mathbb{N}$ is a fixed number, and $\inf _{i, j \geq n} A\left(T^{i} x, T^{j} x\right) \operatorname{or}_{i n f} i_{i, j \geq 1+n k} A\left(T^{i} x, T^{j} x\right)$ or, $\ldots$, or inf $_{i, j \geq k+n k} A\left(T^{i} x, T^{j} x\right)$ converges to $\alpha_{0}, \alpha_{1}, \ldots, \alpha_{k} \geq 0$ respectively implies that $\left\{T^{n}(x)\right\}_{n \in \mathbb{N}}$ or $\left\{T^{n k+1}(x)\right\}_{n \in \mathbb{N}}$ or, $\ldots$, or $\left\{T^{n k+k}(x)\right\}_{n \in \mathbb{N}}$ has a convergent subsequence respectively, and if $A\left(x, T^{k}(x)\right) \geq \alpha_{i}(i=0,1, \ldots, k)$ implies $T^{k}(t)=t$, respectively.

As an immediate application of Theorem 12 we obtain the following form of the monotone principle for fixed apices on topological spaces. 
Theorem 13. (Expansion Monotone Principle for F. A.). Let $T$ be a mapping of a topological space $X:=(X, A)$ into itself, where $X$ satisfies the condition of $k$-inf TCS-convergence. Suppose that there exists a controlling function $B: X \rightarrow \mathbb{R}_{+}^{0}$ such that

$$
A\left(T^{k} x, T^{k} y\right) \geq B(x, y) \geq \inf _{z, r \in \sigma(x, y, \infty)} A(z, r)>0
$$

for all $x, y \in X$, where $x \mapsto A\left(x, T^{k} x\right)$ or $x \mapsto B\left(x, T^{k} x\right)$ is a $T$-orbitally upper semicontinuous function, then $T$ has at least one $k$-fixed apex in $X$.

As an immediate consequence of this statement we obtain the following result for uniqueness fixed apices on topological spaces.

Corollary 34.. Let $T$ be a mapping of a topological space $X:=(X, A)$ into itself, where $X$ satisfies the condition of $k$-inf TCS-convergence. Suppose that there exists a controlling function $B: X \times X \rightarrow \mathbb{R}_{+}^{0}$ such that

$$
A\left(T^{k} x, T^{k} y\right) \geq B(x, y)>A(x, y)
$$

for all $x, y \in X$, or

$$
A\left(T^{k} x, T^{k} y\right)>B(x, y) \geq A(x, y)
$$

for all $x, y \in X$, where $x \mapsto A\left(x, T^{k} x\right)$ or $x \mapsto B\left(x, T^{k} x\right)$ is a T-orbitally upper semicontinuous function. Then $T$ has a unique $k$-fixed apex in $X$.

As an immediate consequence of Theorem 13 we obtain the following global result for $k$-inf TCS-convergence on topological spaces.

Corollary 35.. Let $T$ be a mapping of a topological space $X:=(X, A)$ into itself, where $X$ satisfies the condition of $k$-inf TCS-convergence and for $n \in \mathbb{N} \cup\{0\}$ let

$$
\inf _{i, j \geq n} A\left(T^{i} x, T^{j} x\right)>0 \quad \text { for every } x \in X,
$$

where $x \mapsto A\left(x, T^{k}(x)\right)$ is a T-orbitally upper semicontinuous function, then $T$ has at least one $k$-fixed apex in $X$. If additional there exists a controlling function $B: X \times X \rightarrow \mathbb{R}_{+}^{0}$ such that (25) or (25'), then $T$ has a unique $k$-fixed apex in $X$.

\section{Transversal spaces With the nOnNumerical transverses}

Spring upper ordered spaces. Let $X$ be a nonempty set and let in further $P:=(P, \preccurlyeq)$ be a partially ordered set such that $a, b \in P$ and $a \prec b$. The set (interval) $[a, b)$ is in further defined by

$$
[a, b):=\{t \in P: a \preccurlyeq t \prec b\} .
$$

The function $A: X \times X \rightarrow[a, b) \subset P$ for $a \prec b$ is called an upper spring ordered transverse (or upper spring ordered transversal) on a nonempty set $X$ iff $A(x, y)=a$ if and only if $x=y$ for all $x, y \in X$. 
An upper spring ordered transversal space $X:=(X, A)$ is a nonempty set $X$ together with a given upper spring ordered transverse $A$ on $X$, where every decreasing sequence $\left\{u_{n}\right\}_{n \in \mathbb{N}}$ of elements in $[a, b)$ has a unique element $u \in[a, b)$ as limit (in nottion $u_{n} \rightarrow u(n \rightarrow \infty)$ ). The element $a \in[a, b) \subset P$ is called spring of space $X$.

In 1986 we investigated the concept of upper spring ordered TCS-convergence in a space $X$, i.e., an upper spring ordered transversal space $X:=$ $(X, A)$ satisfies the condition of upper spring ordered TCS-convergence iff $x \in X$ and if $A\left(T^{n}(x), T^{n+1}(x)\right) \rightarrow a(n \rightarrow \infty)$ implies that $\left\{T^{n}(x)\right\}_{n \in \mathbb{N}}$ has a convergent subsequence in $X$, by Tasković [54].

We notice that the sequence $\left\{x_{n}\right\}_{n \in \mathbb{N}}$ in the upper spring ordered transversal space $X:=(X, A)$ is convergent (or upper convergent) in notation $x_{n} \rightarrow x$ $(n \rightarrow \infty)$ iff $A\left(x_{n}, x\right) \rightarrow a$ as $n \rightarrow \infty$; or equivalently, for a decreasing sequence $\left\{a_{n}\right\}_{n \in \mathbb{N}} \in[a, b)$ which converges to $a$ the folowing inequality holds in the form as

$$
A\left(x_{n}, x\right) \prec a_{n} \quad \text { for every } n \in \mathbb{N},
$$

or for $n$ large enough.

On the other hand, in connection with this, the sequence $\left\{x_{n}\right\}_{n \in \mathbb{N}}$ in $X$ will be called upper fundamental (or upper spring fundamental) if the following inequality holds in the form as

$$
A\left(x_{n}, x_{m}\right) \prec a_{n} \text { for all } n, m \in \mathbb{N}(n<m),
$$

or for $n$ and $m$ large enough, where the decreasing sequence $\left\{a_{n}\right\}_{n \in \mathbb{N}}$ in $[a, b)$ converges to $a$.

An upper spring ordered transversal space $X:=(X, A)$ is called upper complete (or upper spring complete) if any upper fundamental sequence $\left\{x_{n}\right\}_{n \in \mathbb{N}}$ in $X$ is upper convergent (to a point of $X$, of course).

On the other hand, an upper spring ordered transversal space $X:=(X, A)$ satisfies the condition of spring sup TCS-convergence iff $x \in X$ and if $\sup _{i, j \geq n} A\left(T^{i} x, T^{j} x\right)$ or $\sup _{i, j \geq 2 n} A\left(T^{i} x, T^{j} x\right)$ or $\sup _{i, j \geq 2 n+1} A\left(T^{i} x, T^{j} x\right)$ converges to $u, v, c \in[a, b)$ respectively implies that $\left\{T^{n}(x)\right\}_{n \in \mathbb{N}}$ or $\left\{T^{2 n}(x)\right\}_{n \in \mathbb{N}}$ or $\left\{T^{2 n+1}(x)\right\}_{n \in \mathbb{N}}$ has a convergent subsequence respectively, and if $A(s, t) \preccurlyeq$ $u, v$, or $c$ implies $s=t$, respectively.

Also, if $T: X \rightarrow X$, then a function $x \mapsto A(x, T(x))$ is ordered $T$ orbitally lower semicontinuous at $\xi \in X$ if $\left\{x_{n}\right\}_{n \in \mathbb{N}}$ is a sequence in $\sigma(x, y, \infty)$ and $x_{n} \rightarrow \xi(n \rightarrow \infty)$ implies that $A(\xi, T(\xi)) \preccurlyeq \lim _{n \rightarrow \infty} A\left(T^{n}(x)\right.$, $\left.T^{n+1}(x)\right)$.

Theorem 14. (Monotone Principle of F.P.). Let $T$ be a mapping of an upper spring ordered transversal space $X:=(X, A)$ into itself, where $X$ satisfies the condition of spring sup TCS-convrgence. Suppose that there exists a controlling function $B: X \times X \rightarrow[a, b)$ for $a \prec b$ such that

$$
A(T x, T y) \preccurlyeq B(x, y) \prec \sup _{z, r \in \sigma(x, y, \infty)} A(z, r) \prec b
$$


for all $x, y \in X$ or

$$
A(T x, T y) \prec B(x, y) \preccurlyeq \sup _{z, r \in \sigma(x, y, \infty)} A(z, r) \prec b
$$

for all $x, y \in X$, where $x \mapsto A(x, T x)$ or $x \mapsto B(x, T x)$ is ordered $T$-orbitally lower semicontinuous, then $T$ has at least one fixed point in $X$. If additional $A(t, t) \preccurlyeq \sup \{A(s, t), A(t, s)\}$ for all $s, t \in X$, then $T$ has a unique fixed point in $X$.

Let $X:=(X, M)$ be a topological space and $T: X \rightarrow X$, where $M: X \rightarrow$ $[a, b) \subset P$. In this part we shall introduce the concept of local sup TCSconvergence in a space $X$, i.e., a topological space $X$ satisfies the condition of local spring sup TCS-convergence iff $x \in X$ and $\sup _{i \geq n} M\left(T^{i} x\right)$ or $\sup _{i \geq 2 n} M\left(T^{i} x\right)$ or $\sup _{i \geq 2 n+1} M\left(T^{n} x\right)$ converges to $u, v, c \succcurlyeq \bar{a}$ respectively implies that $\left\{T^{n}(x)\right\}_{n \in \mathbb{N}}$ or $\left\{T^{2 n}(x)\right\}_{n \in \mathbb{N}}$ or $\left\{T^{2 n+1}(x)\right\}_{n \in \mathbb{N}}$ has a convergent subsequence respectively, and iff $M(t) \preccurlyeq u, v$ or $c$ implies $T(t)=t$, respectively.

We are now in a position to formulate the following our theorem on topological spaces with nonnumerical transverses.

Theorem 15. (Localization Monotone Principle). Let $T$ be a mapping of a topological space $X:=(X, M)$ into itself, where $X$ satisfies the condition of local spring sup TCS-convergence. Suppose that there exists a controlling mapping $N: X \rightarrow[a, b) \subset P$ such that

$$
M(T x) \preccurlyeq N(x) \preccurlyeq \sup _{z \in \sigma(x, \infty)} M(z) \prec b \quad \text { for every } x \in X
$$

where $x \mapsto M(x)$ or $x \mapsto N(x)$ is ordered T-orbitally lower semicontinuous. Then $T$ has at least one fixed point in $X$.

An immediate consequence of the preceding statement is the following result.

Corollary 36.. Let $T$ be a mapping of a topological space $X:=(X, M)$ into itself, where $X$ satisfies the condition of local spring sup TCS-convergence. Suppose that there exists a controlling function $N: X \rightarrow[a, b) \subset P$ such that

$$
M(T x) \preccurlyeq N(x) \preccurlyeq M(x) \quad \text { for every } x \in X,
$$

where $M$ or $N$ is ordered $T$-orbitally lower semicontinuous. Then $T$ has at least one fixed point in $X$.

The proof of this statement is an elementary fact because condition (D') implies condition (D).

Proof of Theorem 15. Let $x \in X$ be an arbitrary point and $n \in \mathbb{N} \cup\{0\}$ be any nonnegative integers. From (D) for $T^{i}(x)$ we have $M\left(T^{i+1} x\right) \preccurlyeq$ 
$N\left(T^{i} x\right) \preccurlyeq \sup _{z \in \sigma\left(T^{i} x, \infty\right)} M(z)$, and hence

$$
\sup _{i \geq n+1} M\left(T^{i} x\right) \preccurlyeq \sup _{i \geq n} N\left(T^{i} x\right) \preccurlyeq \sup _{i \geq n} M\left(T^{i} x\right),
$$

i.e., we obtain that $\left\{\sup _{i \geq n} M\left(T^{i} x\right)\right\}_{n \in \mathbb{N}}$ is a decreasing convergent sequence in $[a, b) \subset P$. This implies (from local spring sup TCS-convergence) that its sequence of iterates $\left\{T^{n}(x)\right\}_{n \in \mathbb{N}}$ contains a convergent subsequence $\left\{T^{n(r)}(x)\right\}_{r \in \mathbb{N}}$ with limit $\zeta \in X$. Since $M: X \rightarrow[a, b) \subset P$ is an ordered $T$-orbitally lower semicontinuous function,

$$
M(\zeta) \preccurlyeq \lim _{r \rightarrow \infty} M\left(T^{n(r)}(x)\right)=\lim _{n \rightarrow \infty} M\left(T^{n}(x)\right)=a
$$

implies that $T(\zeta)=\zeta$. In the cases of other two sequences, in local spring sup TCS-convergence, the proof is a total analogy. Hence the proof in these cases we omit. If the controlling function $N: X \rightarrow[a, b) \subset P$ is ordered $T$-orbitally lower semicontinuous, then from (27) we have the following inequalities

$$
\begin{gathered}
N(\zeta) \preccurlyeq \lim _{r \rightarrow \infty} N\left(T^{n(r)}(x)\right) \preccurlyeq \lim _{n \rightarrow \infty}\left(\sup _{i \geq n} N\left(T^{n}(x)\right)\right. \\
\preccurlyeq \lim _{n \rightarrow \infty}\left(\sup _{i \geq n} M\left(T^{i}(x)\right)=a,\right.
\end{gathered}
$$

which means that $M(T \zeta) \preccurlyeq N(\zeta) \preccurlyeq a$ and thus $T(\zeta)=T(T(\zeta))$, i.e., $T$ has at least one fixed point. The proof is complete.

As an immediate consequence of Theorem 15, in the case of local spring TCS-convergence (i. e., the convergence in $a$ ) we have directly the following statement.

Corollary 37.. Let $T$ be a mapping of a topological space $X:=(X, M)$ into itself, where $X$ satisfies the condition of local spring TCS-convergence. Suppose that there exists a controlling function $N: X \rightarrow[a, b) \subset P$ such that

$$
M(T x) \preccurlyeq N(x) \preccurlyeq \sup _{z \in \sigma(x, \infty)} M(z) \prec b \quad \text { for every } x \in X,
$$

where $x \mapsto M(x)$ or $x \mapsto N(x)$ is ordered T-orbitally lower semicontinuous such that the following inequality holds

$$
\lim _{n \rightarrow \infty}\left(\sup _{i \geq n} N\left(T^{i} x\right)\right) \prec \lim _{n \rightarrow \infty}\left(\sup _{i \geq n} M\left(T^{i} x\right)\right),
$$

then $T$ has at least one fixed point in $X$.

In connection with this for $x, y \in X$ the set $\sigma(x, y, \infty):=\left\{x, y, T x, T y, T^{2} x\right.$, $\left.T^{2} y, \ldots\right\}$ is called the orbit of $x$ and $y$. In this part we begin with a statement which is fundamental for the further considerations. 
Theorem 16. (Corollary of Theorem 15). Let $T$ be a mapping of a topological space $X:=(X, A)$ into itself, where $X$ satisfies the condition of spring sup TCS-convergence. Suppose that there exists a controlling function $B: X \times X \rightarrow[a, b) \subset P$ such that

$$
A(T x, T y) \preccurlyeq B(x, y) \preccurlyeq \sup _{z, r \in \sigma(x, y, \infty)} A(z, r) \prec b
$$

for all $x, y \in X$, where $x \mapsto A(x, T x)$ or $x \mapsto B(x, T x)$ is ordered T-orbitally lower semicontinuous, then $T$ has at least one fixed point in $X$.

The following statement give uniqueness of fixed point on topological spaces with the property of spring sup TCS-convergence.

Corollary 38. Let $T$ be a mapping of a topological space $X:=(X, A)$ into itself, where $X$ satisfies the condition of spring sup TCS-convergence. Suppose that there exists a controlling function $B: X \times X \rightarrow[a, b) \subset P$ such that

$$
A(T x, T y) \preccurlyeq B(x, y) \prec A(x, y) \quad \text { for all } x, y \in X
$$

or

$$
A(T x, T y) \prec B(x, y) \preccurlyeq A(x, y) \quad \text { for all } x, y \in X,
$$

where $x \mapsto A(x, T x)$ or $x \mapsto B(x, T x)$ is ordered T-orbitally lower semicontinuous such that the following inequality holds

$$
\lim _{n \rightarrow \infty}\left(\sup _{i, j \geq n} B\left(T^{i} x, T^{j} x\right)\right) \prec \lim _{n \rightarrow \infty}\left(\sup _{i, j \geq n} A\left(T^{i} x, T^{j} x\right)\right) \prec b
$$

then $T$ has a unique fixed point $\zeta \in X$ and $T^{n}(x) \rightarrow \zeta$ as $n \rightarrow \infty$ for arbitrary $x \in X$.

Theorem 17. (Monotone Principle of F.P.). Let $T$ be a mapping of a topological space $X:=(X, A)$ into itself, where $X$ satisfies the condition of spring sup TCS-convergence. Suppose that there exists a controlling function $B: X \times X \rightarrow[a, b) \subset P$ such that

$$
A(T x, T y) \preccurlyeq B(x, y) \prec \sup _{z, r \in \sigma(x, y, \infty)} A(z, r) \prec b
$$

for all $x, y \in X$, or

$$
A(T x, T y) \prec B(x, y) \preccurlyeq \sup _{z, r \in \sigma(x, y, \infty)} A(z, r) \prec b
$$

for all $x, y \in X$, where $x \mapsto A(x, T x)$ or $x \mapsto B(x, T x)$ is ordered T-orbitally lower semicontinuous, then $T$ has at least one fixed point in $X$. If additional $A(t, t) \preccurlyeq \sup \{A(s, t), A(t, s)\}$ for all $s, t \in X$, then $T$ has a unique fixed point $\zeta \in X$ and $T^{n}(x) \rightarrow \zeta$ as $n \rightarrow \infty$ for arbitrary $x \in X$.

As an immediate consequence of Theorem 17 we obtain the following global result for spring sup TCS-convergence on topological spaces. 
Corollary 39.. Let $T$ be a mapping of a topological space $X:=(X, A)$ into itself, where $X$ satisfies the condition of spring sup TCS-convergence and for $n \in \mathbb{N} \cup\{0\}$ let

$$
\sup _{i, j \geq n} A\left(T^{i} x, T^{j} x\right) \prec b \quad \text { for every } x \in X,
$$

where $x \mapsto A(x, T(x))$ is ordered T-orbitally lower semicontinuous. Then $T$ has at least one fixed point in $X$.

If additional there exists a controlling function $B: X \times X \rightarrow[a, b) \subset P$ such that (31) or (31'), then $T$ has a unique fixed point $\zeta \in X$ and $T^{n}(x) \rightarrow \zeta$ as $n \rightarrow \infty$ for arbitrary $x \in X$.

Also, an immediate consequence of Theorem 17, in the case of spring sup TCS-convergence (i.e., the convergence in $a$ ) we obtain directly the following statement.

Corollary 40.. (Global F.P. Th. for the spring sup TCS-convergence). Let $T$ be a mapping of a topological space $X:=(X, A)$ into itself, where $X$ satisfies the condition of spring sup TCS-convergence, and for $x \in X$ the following fact holds

$$
\sup _{i, j \geq n} A\left(T^{i} x, T^{j} x\right) \rightarrow a \quad(n \rightarrow \infty),
$$

where $x \mapsto A(x, T x)$ is ordered T-orbitally lower semicontinuous function, then $T$ has at least one fixed point in $X$.

If additional there exists a controlling function $B: X \times X \rightarrow[a, b) \subset P$ such that (31) or (31'), then $T$ has a unique fixed point $\zeta \in X$ and $T^{n}(x) \rightarrow \zeta$ as $n \rightarrow \infty$ for arbitrary $x \in X$.

Let, in the next, $X:=(X, M)$ be a topological space and $T: X \rightarrow X$, where $M: X \rightarrow(a, b] \subset P$ is a bounded above function. In this part we shall introduce the concept of local spring inf TCS-convergence in a space $X$, i.e., a topological space $X$ satisfies the condition of local spring inf TCS-convergence iff $x \in X$ and $\inf _{i \geq n} M\left(T^{i} x\right) \operatorname{or}_{\inf _{i \geq 2 n}} M\left(T^{i} x\right)$ or $\inf _{i \geq 2 n+1} M\left(T^{i} x\right)$ converges to $u, v, c \preccurlyeq b$ respectively implies that $\left\{T^{n}(x)\right\}_{n \in \mathbb{N}}$ or $\left\{T^{2 n}(x)\right\}_{n \in \mathbb{N}}$ or $\left\{T^{2 n+1}(x)\right\}_{n \in \mathbb{N}}$ has a convergent subsequence respectively, and if $M(t) \succcurlyeq u, v$, or $c$ implies $T(t)=t$, respectively.

Also, if $T: X \rightarrow X$, then a function $x \mapsto A(x, T(x))$ is ordered $T$ orbitally upper semicontinuous at $\xi \in X$ if $\left\{x_{n}\right\}_{n \in \mathbb{N}}$ is a sequence in $\sigma(x, y, \infty)$ and $x_{n} \rightarrow \xi(n \rightarrow \infty)$ implies that $A(\xi, T(\xi)) \succcurlyeq \lim _{n \rightarrow \infty} A\left(T^{n}(x)\right.$, $\left.T^{n+1}(x)\right)$.

Theorem 18. (Localization Monotone Principle). Let $T$ be a mapping of a topological space $X:=(X, M)$ into itself, where $X$ satisfies the condition of local spring inf TCS-convergence. Suppose that there exists a sontrolling 
function $N: X \rightarrow(a, b] \subset P$ such that

$$
M(T x) \succcurlyeq N(x) \succcurlyeq \inf _{z \in \sigma(x, \infty)} M(z) \succ a \quad \text { for every } x \in X,
$$

where $x \mapsto M(x)$ or $x \mapsto N(x)$ is ordered T-orbitally upper semicontinuous. Then $T$ has at least one fixed point in $X$.

The proof of this statement is a total analogy with the former proof of Theorem 15. Thus the proof we omit.

An immediate consequence of the preceding statement is the following result.

Corollary 41.. Let $T$ be a mapping of a topological space $X:=(X, M)$ into itself, where $X$ satisfies the condition of local spring inf TCS-convergence. Suppose that there exists a controlling function $N: X \rightarrow(a, b] \subset P$ such that

$$
M(T x) \succcurlyeq N(x) \succcurlyeq M(x) \quad \text { for every } x \in X,
$$

where $M$ or $N$ is ordered T-orbitally upper semicontinuous. Then $T$ has at least one fixed point in $X$.

The proof of this statement is an elementary fact because condition $\left(G^{\prime}\right)$ implies condition $(G)$.

Corollary 42.. Let $T$ be a mapping of a topological space $X:=(X, M)$ into itself, where $X$ satisfies the condition of local spring inf TCS-convergence, and for $n \in \mathbb{N} \cup\{0\}$ let

$$
\inf _{i \geq n} M\left(T^{i}(x)\right) \succ a \quad \text { for every } x \in X,
$$

where $x \mapsto M(x)$ is ordered T-orbitally upper semicontinuous. Then $T$ has at least one fixed point in $X$.

The proof of this statement is a total analogous with the former proof of Corollary 40. Thus proof we omit.

Uniqueness of fixed point. Let $X:=(X, A)$ be a topological space and $T: X \rightarrow X$, where $A: X \times X \rightarrow(a, b] \subset P$. In this part we shall introduce the concept of spring inf TCS-convergence in a space $X$, i.e., a topological space $X$ satisfies the condition of spring inf TCS-convergence iff $x \in X$

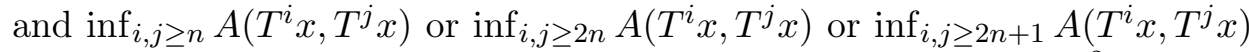
converges to $u, v, c \preccurlyeq b$ respectively implies that $\left\{T^{n}(x)\right\}_{n \in \mathbb{N}}$ or $\left\{T^{2 n}(x)\right\}_{n \in \mathbb{N}}$ or $\left\{T^{2 n+1}(x)\right\}_{n \in \mathbb{N}}$ has a convergent subsequence respectively, and if $A(s, t) \succcurlyeq$ $u, v$ or $c$ implies $s=t$, respectively.

Theorem 19. (Monotony General Expansion.) Let $T$ be a mapping of a topological space $X:=(X, A)$ into itself, where $X$ satisfies the condition of 
spring inf TCS-convergence. Suppose that there exists a controlling function $B: X \times X \rightarrow(a, b] \subset P$ such that

$$
A(T x, T y) \succcurlyeq B(x, y) \succ \inf _{z, r \in \sigma(x, y, \infty)} A(z, r) \succ a
$$

for all $x, y \in X$, or

$$
A(T x, T y) \succ B(x, y) \succcurlyeq \inf _{z, r \in \sigma(x, y, \infty)} A(z, r) \succ a
$$

for all $x, y \in X$, where $x \mapsto A(x, T x)$ or $x \mapsto B(x, T x)$ is ordered $T$ orbitally upper semicontinuous, then $T$ has at lest one fixed point in $X$. If $A(t, t) \succcurlyeq \inf \{A(t, s), A(s, t)\}$ for all $s, t \in X$, then $T$ has a unique fixed point $\zeta \in X$ and $T^{n}(x) \rightarrow \zeta$ as $n \rightarrow \infty$ for arbitrary $x \in X$.

The proof of this statement is a total analogy with the former proof of Theorem 17. Thus the proof we omit.

As an immediate consequence of Theorem 19 directly we obtain the following statement on topological spaces.

Corollary 43.. (Global General Expansion). Let $T$ be a mapping of a topological space $X:=(X, A)$ into itself, where $X$ satisfies the condition of spring inf TCS-convergence and for $n \in \mathbb{N} \cup\{0\}$ let

$$
\inf _{i, j \geq n} A\left(T^{i} x, T^{j} x\right) \succ a \quad \text { for every } x \in X,
$$

where $x \mapsto A(x, T(x))$ is ordered T-orbitally upper semicontinuous function. Then $T$ has at least one fixed point in $X$. In additional there exists a controlling function $B: X \times X \rightarrow(a, b] \subset P$ such that

$$
A(T x, T y) \succcurlyeq B(x, y) \succ A(x, y) \quad \text { for all } x, y \in X
$$

or

$$
A(T x, T y) \succ B(x, y) \succcurlyeq A(x, y) \quad \text { for all } x, y \in X,
$$

then $T$ has a unique fixed point $\zeta \in X$ and $T^{n}(x) \rightarrow \zeta$ as $n \rightarrow \infty$ for arbitrary $x \in X$.

Let $X:=(X, M)$ be a topological space and $T: X \rightarrow X$, where $M$ : $X \rightarrow[a, b) \subset P$. In this part we shall introduce the concept of local spring $k$-sup TCS-convergence in a space $X$, i.e., a topological space $X$ satisfies the condition of local spring $k$-sup TCS-convergence iff $x \in X, k \in$ $\mathbb{N}$ is a fixed number, and $\sup _{i \geq n} M\left(T^{i} x\right)$ or $\sup _{i \geq 1+n k} M\left(T^{i} x\right)$ or, $\ldots$, or $\sup _{i \geq k+n k} M\left(T^{i} x\right)$ converges to $\alpha_{0}, \alpha_{1}, \ldots, \alpha_{k} \succcurlyeq a$ respectively implies that $\left\{T^{n}(x)\right\}_{n \in \mathbb{N}}$ or $\left\{T^{n k+1}(x)\right\}_{n \in \mathbb{N}}$ or, $\ldots$, or $\left\{T^{n k+k}(x)\right\}_{n \in \mathbb{N}}$ has a convergent subsequence respectively, and if $M\left(T^{k}(t)\right) \preccurlyeq \alpha_{i}(i=0,1, \ldots, k)$ implies $T^{k}(t)=t$, respectively.

We are now in a position to formulate the following our theorem on topological spaces for the fixed apices. 
Theorem 20. (Localization Monotone Principle for F. A.). Let $T$ be a mapping of a topological space $X:=(X, M)$ into itself, where $X$ satisfies the condition of local spring $k$-sup TCS-convergence. Suppose that there exists a controlling function $N: X \rightarrow[a, b) \subset P$ such that

$$
M\left(T^{k}(x)\right) \preccurlyeq N(x) \preccurlyeq \sup _{z \in \sigma(x, \infty)} M(z) \prec b \quad \text { for every } x \in X,
$$

where $x \mapsto M\left(T^{k}(x)\right)$ or $x \mapsto N\left(T^{k}(x)\right)$ is ordered $T$-orbitally lower semicontinuous. Then $T$ has at least one fixed $k$-apex in $X$.

As an immediate consequence of this statement we have the following result for fixed apices on topological spaces.

Corollary 44.. Let $T$ be a mapping of a topological space $X:=(X, M)$ into itself, where $X$ satisfies local spring $k$-sup TCS-convergence. Suppose that there exists a controlling function $N: X \rightarrow[a, b) \subset P$ such that

$$
M\left(T^{k}(x)\right) \preccurlyeq N(x) \preccurlyeq M(x) \quad \text { for every } x \in X,
$$

where $x \mapsto M\left(T^{k}(x)\right)$ or $x \mapsto N\left(T^{k}(x)\right)$ is ordered $T$-orbitally lower semicontinuous. Then $T$ has at least one fixed $k$-apex in $X$.

Corollary 45.. (Local Form of $k$-Global F. A. Th.). Let $T$ be a mapping of a topological space $X:=(X, M)$ into itself, where $X$ satisfies the condition of local spring $k$-sup TCS-convergence, and for $n \in \mathbb{N} \cup\{0\}$ let

$$
\sup _{i \geq n} M\left(T^{i}(x)\right) \prec b \quad \text { for every } x \in X,
$$

where $x \mapsto M\left(T^{k}(x)\right)$ is ordered T-orbitally lower semicontinuous, then $T$ has at least one fixed $k$-fork in $X$.

The proof of this statement is a total analogy with the former proof of Corollary 9. Thus the proof we omit.

Let $X:=(X, A)$ be a topological space and $T: X \rightarrow X$, where $A$ : $X \times X \rightarrow[a, b) \subset P$. In this part we shall introduce the concept of spring $k$-sup TCS-convergence in a space $X$, i.e., a topological space $X$ satisfies the condition of spring $k$-sup TCS-convergence iff $x \in X, k \in \mathbb{N}$ is a fixed number, and $\sup _{i, j \geq n} A\left(T^{i} x, T^{j} x\right)$ or $\sup _{i, j \geq 1+n k} A\left(T^{i} x, T^{j} x\right)$ or, $\ldots$, or $\sup _{i, j \geq k+n k} A\left(T^{i} x, T^{j} x\right)$ converges to $\alpha_{0}, \alpha_{1}, \ldots, \alpha_{k} \succcurlyeq a$ respectively implies that $\left\{T^{n}(x)\right\}_{n \in \mathbb{N}}$ or $\left\{T^{n k+1}(x)\right\}_{n \in \mathbb{N}}$ or, $\ldots$, or $\left\{T^{n k+k}(x)\right\}_{n \in \mathbb{N}}$ has a convergent subsequence respectively, and if $A\left(x, T^{k}(x)\right) \preccurlyeq \alpha_{i}(i=0,1, \ldots, k)$ implies $T^{k}(t)=t$, respectively.

As an immediate application of Theorem 20 we obtain the following form of the monotone principle for fixed apices on topological spaces.

Theorem 21. (Monotone Principle of F. A.). Let $T$ be a mapping of a topological space $X:=(X, A)$ into itself, where $X$ satisfies the condition 
of spring k-sup TCS-convergence. Suppose that there exists a controlling function $B: X \times X \rightarrow[a, b) \subset P$ such that

$$
A\left(T^{k} x, T^{k} y\right) \preccurlyeq B(x, y) \preccurlyeq \sup _{z, r \in \sigma(x, y, \infty)} A(z, r) \prec b
$$

for all $x, y \in X$, where $x \mapsto A\left(x, T^{k} x\right)$ or $x \mapsto B\left(x, T^{k} x\right)$ is ordered $T$ orbitally lower semicontinuous, then $T$ has at least one $k$-fixed apex in $X$.

As an immediate consequence of this statement we obtain the following result for uniqueness fixed apices on topological spaces.

Corollary 46.. Let $T$ be a mapping of a topological space $X:=(X, A)$ into itself, where $X$ satisfies spring $k$-sup TCS-convergence. Suppose that there exists a controlling function $B: X \times X \rightarrow[a, b) \subset P$ such that

$$
A\left(T^{k}(x), T^{k}(y) \preccurlyeq B(x, y) \prec A(x, y)\right.
$$

for all $x, y \in X$, or

$$
A\left(T^{k}(x), T^{k}(y)\right) \prec B(x, y) \preccurlyeq A(x, y)
$$

for all $x, y \in X$, where $x \mapsto A\left(x, T^{k} x\right)$ or $x \mapsto B\left(x, T^{k} x\right)$ is ordered $T$ orbitally lower semicontinuous. Then $T$ has a unique $k$-fixed apex in $X$.

Corollary 47.. Let $T$ be a mapping of a topological space $X:=(X, A)$ into itself, where $X$ satisfies the condition of spring $k$-sup TCS-convergence and for $n \in \mathbb{N} \cup\{0\}$ let

$$
\sup _{i, j \geq n} A\left(T^{i} x, T^{j} x\right) \prec b \quad \text { for every } x \in X,
$$

where $x \mapsto A\left(x, T^{k} x\right)$ is ordered T-orbitally lower semicontinuous. Then $T$ has at least one $k$-fixed apex in $X$.

If additional there exists a controlling function $B: X \times X \rightarrow[a, b) \subset P$ such that (35) or (35'), then $T$ has a unique $k$-fixed apex in $X$.

Also, as an immediate consequence of Theorem 21, in the case of spring sup TCS-convergence (i.e, the convergence in $a$ ), we obtain directly the following result.

Corollary 48.. Let $T$ be a mapping of a topological space $X:=(X, A)$ into itself, where $X$ satisfies the condition of spring sup TCS-convergence and for $x \in X$ the following fact holds

$$
\sup _{i, j \geq n} A\left(T^{i} x, T^{j} x\right) \rightarrow a \quad(n \rightarrow \infty),
$$

where $x \mapsto A\left(x, T^{k}(x)\right)$ is ordered T-orbitally lower semicontinuous, then $T$ has at least one $k$-fixed apex in $X$.

If additional there exist a controlling function $B: X \times X \rightarrow[a, b) \subset P$ such that (35) or (35'), then $T$ has a unique $k$-fixed apex in $X$. 
Let $X:=(X, M)$ be a topological space and $T: X \rightarrow X$, where $M$ : $X \rightarrow(a, b] \subset P$. In this part we shall introduce the concept of local spring $k$-inf TCS-convergence in a space $X$, i.e, a topological space $X$ satisfies the condition of local spring $k$-inf TCS-convergence iff $x \in X$, $k \in \mathbb{N}$ is a fixed number, and $\inf _{i \geq n} M\left(T^{i} x\right)$ or $\inf _{i \geq 1+n k} M\left(T^{i} x\right)$ or, $\ldots$, or $\inf _{i \geq k+n k} M\left(T^{i} x\right)$ converges to $\alpha_{0}, \alpha_{1}, \ldots, \alpha_{k} \preccurlyeq b$ respectively implies that $\left\{T^{n}(x)\right\}_{n \in \mathbb{N}}$ or $\left\{T^{n k+1}(x)\right\}_{n \in \mathbb{N}}$ or, $\ldots$, or $\left\{T^{n k+k}(x)\right\}_{n \in \mathbb{N}}$ has a convergent subsequence respectively, and if $M\left(T^{k}(t)\right) \succcurlyeq \alpha_{i}(i=0,1, \ldots, k)$ implies $T^{k}(t)=t$, respectively.

We are now in a position to formulate the following our theorem on topological spaces for fixed apices.

Theorem 22. (Localization of General Expansion for F. A.). Let $T$ be a mapping of a topological space $X:=(X, M)$ into itself, where $X$ satisfies the condition of local spring $k$-inf TCS-convergence. Suppose that there exists a controlling function $N: X \rightarrow(a, b] \subset P$ such that

$$
M\left(T^{k}(x)\right) \succcurlyeq N(x) \succcurlyeq \inf _{z \in \sigma(x, \infty)} M(z) \succ a \quad \text { for every } x \in X,
$$

where $x \mapsto M\left(T^{k}(x)\right)$ or $x \mapsto N\left(T^{k}(x)\right)$ is ordered T-orbitally upper semicontinuous. Then $T$ has at least one $k$-fixed apex in $X$.

As an immediate consequence of this statement we have the following result for fixed apices on topological space.

Corollary 49.. Let $T$ be a mapping of a topological space $X:=(X, M)$ into itself, where $X$ satisfies the condition of local spring $k$-inf TCS-convergence. Suppose that there a controlling function $N: X \rightarrow(a, b] \subset P$ such that

$$
M\left(T^{k}(x)\right) \succcurlyeq N(x) \succcurlyeq M(x) \quad \text { for every } x \in X,
$$

where $x \mapsto M\left(T^{k} x\right)$ or $x \mapsto N\left(T^{k} x\right)$ is ordered T-orbitally upper semicontinuous. Then $T$ has at least one $k$-fixed apex in $X$.

As an immediate consequence of the preceding two results directly we obtain a global statement on topological spaces.

Corollary 50.. Let $T$ be a mapping of a topological space $X:=(X, M)$ into itself, where $X$ satisfies the condition of local spring $k$-inf TCS-convergence, and for $n \in \mathbb{N} \cup\{0\}$ let

$$
\inf _{i \geq n} M\left(T^{i} x\right) \succ a \quad \text { for every } x \in X,
$$

where $x \mapsto M\left(T^{k}(x)\right)$ is ordered $T$-orbitally upper semicontinuous. Then $T$ has at least one $k$-fixed apex in $X$.

Let $X:=(X, A)$ be a topological space and $T: X \rightarrow X$, where $A$ : $X \times X \rightarrow(a, b] \subset P$. In this part we shall introduce the concept of spring 
$k$-inf TCS-convergence in a space $X$, i.e., a topological space $X$ satisfies the condition of spring $k$-inf TCS-convergence iff $x \in X, k \in \mathbb{N}$ is a fixed number, and $\inf _{i, j \geq n} A\left(T^{i} x, T^{j} x\right)$ or inf $_{i, j \geq 1+n k} A\left(T^{i} x, T^{j} x\right)$ or, $\ldots$, or $\inf _{i, j \geq k+n k} A\left(T^{i} x, T^{j} x\right)$ converges to $\alpha_{0}, \alpha_{1}, \ldots, \alpha_{k} \preccurlyeq b$ respectively implies that $\left\{T^{n}(x)\right\}_{n \in \mathbb{N}}$ or $\left\{T^{n k+1}(x)\right\}_{n \in \mathbb{N}}$ or,$\ldots$, or $\left\{T^{n k+k}(x)\right\}_{n \in \mathbb{N}}$ has a convergent subsequence respectively, and if $A\left(x, T^{k}(x)\right) \succcurlyeq \alpha_{i}(i=0,1, \ldots, k)$ implies $T^{k}(t)=t$, respectively.

As an immediate application of Theorem 22 we obtain the following form of the monotone principle for fixed apices on topological spaces.

Theorem 23. (Expansion Monotone Principle for F. A.). Let $T$ be a mapping of a topological space $X:=(X, A)$ into itself, where $X$ satisfies the condition of spring $k$-inf TCS-convergence. Suppose that there exists a controlling function $B: X \rightarrow(a, b] \subset P$ such that

$$
A\left(T^{k} x, T^{k} y\right) \succcurlyeq B(x, y) \succcurlyeq \inf _{z, r \in \sigma(x, y, \infty)} A(z, r) \succ a
$$

for all $x, y \in X$, where $x \mapsto A\left(x, T^{k} x\right)$ or $x \mapsto B\left(x, T^{k} x\right)$ is ordered $T$ orbitally upper semicontinuous, then $T$ has at least one $k$-fixed apex in $X$.

As an immediate consequence of this statement we obtain the following result for uniqueness fixed apices on topological spaces.

Corollary 51.. Let $T$ be a mapping of a topological space $X:=(X, A)$ into itself, where $X$ satisfies the condition of spring $k$-inf TCS-convergence. Suppose that there exists a controlling function $B: X \times X \rightarrow(a, b] \subset P$ such that

$$
A\left(T^{k} x, T^{k} y\right) \succcurlyeq B(x, y) \succ A(x, y)
$$

for all $x, y \in X$, or

$$
A\left(T^{k} x, T^{k} y\right) \succ B(x, y) \succcurlyeq A(x, y)
$$

for all $x, y \in X$, where $x \mapsto A\left(x, T^{k} x\right)$ or $x \mapsto B\left(x, T^{k} x\right)$ is ordered $T$ orbitally upper semicontinuous. Then $T$ has a unique $k$-fixed apex in $X$.

As an immediate consequence of Theorem 23 we obtain the following global result for spring $k$-inf TCS-convergence on topological spaces.

Corollary 52.. Let $T$ be a mapping of a topological space $X:=(X, A)$ into itself, where $X$ satisfies the condition of spring $k$-inf TCS-convergence and for $n \in \mathbb{N} \cup\{0\}$ let

$$
\inf _{i, j \geq n} A\left(T^{i} x, T^{j} x\right) \succ a \quad \text { for every } x \in X,
$$

where $x \mapsto A\left(x, T^{k}(x)\right)$ is ordered T-orbitally upper semicontinuous, then $T$ has at least one $k$-fixed apex in $X$.

If additional there exists a controlling function $B: X \times X \rightarrow(a, b] \subset P$ such that (37) or (37'), then $T$ has a unique $k$-fixed apex in $X$. 
Spring lower ordered spaces. Let $X$ be a nonempty set and let $P:=$ $(P, \preccurlyeq)$ be a partially ordered set such that $a, b \in P$ and $a \prec b$. The set (interval) $(a, b]$ is defined by

$$
(a, b]:=\{t \in P: a \prec t \preccurlyeq b\} .
$$

The function $A: X \times X \rightarrow(a, b] \subset P$ for $a \prec b$ is called a lower spring ordered transverse (or lower spring ordered transversal) on a nonempty set $X$ iff $A(x, y)=b$ if and only if $x=y$ for all $x, y \in X$.

A lower spring ordered transversal space $X:=(X, A)$ is a nonempty set $X$ together with a given lower spring ordered transverse $A$ on $X$, where every increasing sequence $\left\{u_{n}\right\}_{n \in \mathbb{N}}$ of elements in $(a, b]$ has a unique element $u$ in $(a, b]$ as limit (in nottion $u_{n} \rightarrow u(n \rightarrow \infty)$ ). The element $b \in(a, b] \subset P$ is called spring of space $X$.

In 1986 we investigated the concept of lower spring ordered TCS-convergence in a space $X$, i.e., a lower spring ordered transversal space $X:=(X, A)$ satisfies the condition of lower spring ordered TCS-convergence iff $x \in X$ and if $A\left(T^{n}(x), T^{n+1}(x)\right) \rightarrow b(n \rightarrow \infty)$ implies that $\left\{T^{n}(x)\right\}_{n \in \mathbb{N}}$ has a convergent subsequence in $X$, by Tasković [54].

We notice that the sequence $\left\{x_{n}\right\}_{n \in \mathbb{N}}$ in the lower spring ordered transversal space $X:=(X, A)$ is convergent (or lower convergent) in notation $x_{n} \rightarrow x$ $(n \rightarrow \infty)$ iff $A\left(x_{n}, x\right) \rightarrow b$ as $n \rightarrow \infty$; or equivalently for an increasing sequence $\left\{b_{n}\right\}_{n \in \mathbb{N}} \in(a, b]$ which converges to $b$ the folowing inequality holds in the form as

$$
A\left(x_{n}, x\right) \succ b_{n} \quad \text { for every } n \in \mathbb{N},
$$

or for $n$ large enough.

On the other hand, in connection with this, the sequence $\left\{x_{n}\right\}_{n \in \mathbb{N}}$ in $X$ will be called lower fundamental (or lower spring fundamental) if the following inequality holds in the form as

$$
A\left(x_{n}, x_{m}\right) \succ b_{n} \quad \text { for all } n, m \in \mathbb{N}(n<m),
$$

or for $n$ and $m$ large enough, where the increasing sequence $\left\{b_{n}\right\}_{n \in \mathbb{N}} \in(a, b]$ converges to $b$.

A lower spring ordered transversal space $X:=(X, A)$ is called lower complete (or lower spring complete) if any lower fundamental sequence $\left\{x_{n}\right\}_{n \in \mathbb{N}}$ in $X$ is lower convergent (to a point of $X$, of course).

On the other hand, a lower spring ordered transversal space $X:=(X, A)$ satisfies the condition of spring inf TCS-convergence iff $x \in X$ and if $\inf _{i, j \geq n} A\left(T^{i} x, T^{j} x\right)$ or $\inf _{i, j \geq 2 n} A\left(T^{i} x, T^{j} x\right)$ or $\inf _{i, j \geq 2 n+1} A\left(T^{i} x, T^{j} x\right)$ converges to $u, v, c \in(a, b]$ respectively implies that $\left\{T^{n}(x)\right\}_{n \in \mathbb{N}}$ or $\left\{T^{2 n}(x)\right\}_{n \in \mathbb{N}}$ or $\left\{T^{2 n+1}(x)\right\}_{n \in \mathbb{N}}$ has a convergent subsequence respectively, and if $A(s, t) \succcurlyeq$ $u, v$, or $c$ implies $s=t$, respectively.

Theorem 24. (Monotone Principle of F.P.). Let $T$ be a mapping of a lower spring ordered transversal space $X:=(X, A)$ into itself, where $X$ satisfies 
the condition of spring inf TCS-convrgence. Suppose that there exists a controlling function $B: X \times X \rightarrow(a, b]$ for $a \prec b$ such that

$$
A(T x, T y) \succcurlyeq B(x, y) \succ \inf _{z, r \in \sigma(x, y, \infty)} A(z, r) \succ a
$$

for all $x, y \in X$ or

$$
A(T x, T y) \succ B(x, y) \succcurlyeq \inf _{z, r \in \sigma(x, y, \infty)} A(z, r) \succ a
$$

for all $x, y \in X$, where $x \mapsto A(x, T x)$ or $x \mapsto B(x, T x)$ is ordered T-orbitally upper semicontinuous, then $T$ has at least one fixed point in $X$.

If additional $A(t, t) \succcurlyeq \inf \{A(s, t), A(t, s)\}$ for all $s, t \in X$, then $T$ has a unique fixed point in $X$.

Asymptotic conditions on spring ordered spaces. We are now in a position to formulate first the following our theorem on upper spring ordered spaces.

Theorem 25. Let $T$ be a mapping of upper spring ordered transversal space $X:=(X, A)$ into itself, where $X$ satisfies the condition of upper spring ordered TCS-convergence. Suppose that for all $x, y \in X$ there exist a sequence of functions $\left\{\alpha_{n}(x, y)\right\}_{n \in \mathbb{N}}$ such that $\alpha_{n}(x, y) \rightarrow a(n \rightarrow \infty)$ and positive integer $m(x, y)$ such that

$$
A\left(T^{n}(x), T^{n}(y)\right) \preccurlyeq \alpha_{n}(x, y) \quad \text { for all } n \geq m(x, y),
$$

where $x \mapsto A(x, T(x))$ is ordered lower semicontinuous, then $T$ has a unique fixed point $\xi \in X$ and $T^{n}(x) \rightarrow \xi(n \rightarrow \infty)$ for each $x \in X$.

A proof of this statement may be found in: Taskovi ć [51]. For second proof of this statement see: Tasković [37].

We notice that, from the preceding facts of this paper, we can give the following local form of this statement.

Theorem 26. (Localization of $(\mathrm{R})$ ). Let $\mathrm{T}$ be a mapping of upper spring ordered transversal space $X:=(X, A)$ into itself, where $X$ satisfies the condition of upper spring ordered TCS-convergence. Suppose that for each $x \in X$ there exist a sequence of functions $\left\{\alpha_{n}(x, T(x))\right\}_{n \in \mathbb{N}}$ such that $\alpha_{n}(x, T(x)) \rightarrow$ $a(n \rightarrow \infty)$ and positive integer $m(x, T(x))$ such that

$$
A\left(T^{n}(x), T^{n+1}(x)\right) \preccurlyeq \alpha_{n}(x, T(x)) \text { for all } n \geq m(x, T(x)),
$$

where $x \mapsto A(x, T(x))$ is ordered lower semicountinuous, then $T$ has at least one fixed point in $X$.

In the next, we are now in a position to formulate the following our statement on lower spring ordered spaces.

Theorem 27. Let $T$ be a mapping of lower spring ordered transversal space $X:=(X, A)$ into itself, where $X$ satisfies the condition of lower spring ordered TCS-convergence. Suppose that for all $x, y \in X$ there exist a sequence 
of functions $\left\{\alpha_{n}(x, y)\right\}_{n \in \mathbb{N}}$ such that $\alpha_{n}(x, y) \rightarrow b(n \rightarrow \infty)$ and positive integer $m(x, y)$ such that

$$
A\left(T^{n}(x), T^{n}(y)\right) \succcurlyeq \alpha_{n}(x, y) \text { for all } n \geq m(x, y),
$$

where $x \mapsto A(x, T(x))$ is ordered upper semicontinuous, then $T$ has a unique fixed point $\xi \in X$ and $T^{n}(x) \rightarrow \xi(n \rightarrow \infty)$ for each $x \in X$.

A proof of this statement may be found in: Tas k ovi ć [51]. For second proof of this statement see: Taskovi ć [37].

We notice that, from the preceding facts of this paper, we can give the following local form of this statement.

Theorem 28. (Localization of $(\mathrm{G})$ ). Let $T$ be a mapping of lower spring ordered transversal space $X:=(X, A)$ into itself, where $X$ satisfies the condition of lower spring ordered TCS-convergence. Suppose that for each $x \in X$ there exist a sequence of functions $\left\{\alpha_{n}(x, T(x))\right\}_{n \in \mathbb{N}}$ such that $\alpha_{n}(x, T(x)) \rightarrow$ $b(n \rightarrow \infty)$ and positive integer $m(x, T(x))$ such that

$$
A\left(T^{n}(x), T^{n+1}(x)\right) \succcurlyeq \alpha_{n}(x, T(x)) \text { for all } n \geq m(x, T(x)),
$$

where $x \mapsto A(x, T(x))$ is ordered upper semicountinuous, then $T$ has at least one fixed point in $X$.

We notice that a middle spring ordered transversal space $X$ is an upper spring ordered transversal space and a lower spring ordered transversal space simultaneous. For further facts on middle spring ordered transversal spaces see: Task ović [51].

This paper continues the study of the Transversal Chaos Spaces (upper, lower and middle) which have been introduced in mathematics in 1998 by M. R. Task ović. The aim of this paper is to provide some characterizations of a common fixed point for three maps on a transversal parametric spring spaces (upper, lower and middle) with the property of HCS-convergence.

This concept have very important applications in numerical analysis and quantum particle physics by L. Collatz [Funktionalanalysis und Num. Math., Springer - Verlag, Berlin 1964] and El. N a sh i e [A review of E-infinity theory and the mass spectrum of high energy particle physics. Chaos, Solitons \& Fractals 2004; 19: 209-36].

We notice that the upper transversal parametric spaces have been introduced in 1998 by Tasković [46]. For further facts on upper transversal parametric chaos spaces see: Tasković [51].

Let $X$ be a nonempty set, $P:=(P, \preccurlyeq)$ be a partially ordered set, and let $\mathbb{S}$ be a totally ordered set.

In connection with the preceding facts, the function $M: X \times X \times \mathbb{S} \rightarrow$ $[a, b] \subset P($ or $M: X \times X \times \mathbb{S} \rightarrow[a, b) \subset P)$ for $a \prec b$ is called an upper parametric spring transverse on $X$ (or upper parametric spring transver$s a l)$ iff: there is $c \in \mathbb{S}$ such that $M(u, v, t)=a$ for every $t \succ c \succcurlyeq a$ if and 
only if $u=v$, and $M(u, v, t) \rightarrow a$ as $t \rightarrow \sup \mathbb{S}($ or $M(u, v, t)=M(u, v, s)$ and $t \neq s$ implies $u=v$ ) for all $u, v \in X$.

An upper transversal parametric spring space is a set $X$ together with a given upper parametric spring transverse $M: X \times X \times \mathbb{S} \rightarrow[a, b]$ for some $a \prec b$ in notation $X:=(X, M)$. The element $a \in[a, b)$ is called spring of space $X$.

Otherwise, the function $M$ is called a semiupper parametric spring transverse on $X$ (or semiupper parametric spring transversal) iff: there is $c \in \mathbb{S}$ such that $M(u, v, t)=a$ for every $t \succ c \succcurlyeq a$ implies $u=v$, and $M(u, v, t) \rightarrow a$ as $t \rightarrow \sup \mathbb{S}$ (or $M(u, v, t)=M(u, v, s)$ and $t \neq s$ implies $u=v$ ) for all $u, v \in X$. A semiupper transversal parametric spring space $X:=(X, M)$ is a set $X$ together with a given semiupper parametric spring transverse on $X$.

Let $X:=(X, M)$ be an upper transversal parametric spring space. For $S \subset X$ we denoted tpc. $\operatorname{diam}(S)$ as a transversal parametric spring diameter of $S$, in the sense that

$$
\operatorname{tpc} \cdot \operatorname{diam}(S):=\sup \{M(x, y, t): x, y \in S\},
$$

where $t \in \mathbb{S}$ and $S \subset Y$ implies tpc. $\operatorname{diam}(S) \preccurlyeq \operatorname{tpc} \operatorname{diam}(Y)$.

Elements of an upper parametric spring transversal space will usually be called points. Given an upper parametric spring transversal space $X:=$ $(X, M)$ and a point $z \in X$, the open ball of center $z$ and radius $r$ is the set

$$
M(B(z, r)):=\{x \in X: M(z, x, t) \prec r\},
$$

where $t \in \mathbb{S}$. The upper parametric spring convergence $x_{n} \rightarrow x$ as $n \rightarrow \infty$ in the upper transversal parametric spring space $X:=(X, M)$ means that the following fact holds that

$$
M\left(x_{n}, x, t\right) \rightarrow a \quad(\text { for } t \in \mathbb{S}) \quad \text { as } \quad n \rightarrow \infty .
$$

The sequence $\left\{x_{n}\right\}_{n \in \mathbb{N}}$ in the upper transversal parametric spring space $X:=(X, M)$ is called upper transversal parametric spring sequence (or upper parametric spring Cauchy sequence) iff: for every decreasing sequence $\left\{a_{n}\right\}_{n \in \mathbb{N}}$ which converge to $a$ there is an $n_{0}=n_{0}(\varepsilon)$ such that

$$
M\left(x_{n}, x_{m}, t\right) \prec a_{n} \text { for all } n, m \geq n_{0} .
$$

Let $X$ be an upper transversal parametric spring space. We notice, from Tasković [54], that a sequence $\left\{x_{n}\right\}_{n \in \mathbb{N}}$ in $X$ is said to be upper transversal parametric spring sequence if and only if

$$
\lim _{n \rightarrow \infty}\left(\operatorname{tpc} \operatorname{diam}\left\{x_{k}: k \geq n\right\}\right)=a .
$$

In this sense, an upper transversal parametric spring space is called upper parametric spring complete iff every upper transversal parametric spring sequence upper parametric spring converges. Also, a space $X:=(X, M)$ is said to be upper parametric spring orbitally complete (or upper 
parametric spring T-orbitally complete) iff every upper parametric spring transversal sequence which contained in $\mathcal{O}(x):=\left\{x, T x, T^{2} x, \ldots\right\}$ for some $x \in X$ upper parametric spring converges in $X$.

Also, for the convergence of a sequence $\left\{x_{n}\right\}_{n \in \mathbb{N}}$ in the upper transversal parametric spring spaces see: Tasković [54, p. 87, 88 and 89].

Let $X$ be an upper transversal parametric spring space. We shall consider the concept of HCS-convergence in a space $X$ by Tasković [51]; i.e., an upper transversal parametric spring space $X$ satisfies the condition of HCS-convergence iff $\left\{x_{n}\right\}_{n \in \mathbb{N}}$ is a sequence in $X$ and if the convergence of the sequence $\left\{M\left(x_{n}, x_{n+1}, t\right)\right\}_{n \in \mathbb{N}}$ implies that $\left\{x_{n}\right\}_{n \in \mathbb{N}}$ has two convergent subsequences $\left\{x_{2 n(r)}\right\}_{r \in \mathbb{N}}$ and $\left\{x_{2 n(r)+1}\right\}_{r \in \mathbb{N}}$ to a point $\xi \in X$. For this form of convergence on topological spaces see: Tasković [37, p. 61]. For another convergence (in the classical sense) on the upper transversal parametric spaces see: Tasković [50].

Also, let $f$ and $h$ be self maps on a transversal upper parametric spring space $X$. They are compatible if $\lim _{n \rightarrow \infty} f h\left(x_{n}\right)=\lim _{n \rightarrow \infty} h f\left(x_{n}\right)$, whenever $\left\{x_{n}\right\}_{n \in \mathbb{N}}$ is a sequence in $X$ such that $\lim _{n \rightarrow \infty} f\left(x_{n}\right)=\lim _{n \rightarrow \infty} h\left(x_{n}\right)=$ $\xi$ for some point $\xi \in X$.

On the other hand, the mappings $f$ and $h$ from a transversal upper parametric spring space $X$ into itself are weakly compatible if they commute at their coincidence point, i.e., $f(\xi)=h(\xi)$ implies that $f h(\xi)=h f(\xi)$. We notice that a pair $\{f, h\}$ of compatible maps is weakly compatible, but converse is not true in general.

We are now in a position to formulate the following statement, as a consequence of the preceding facts, on upper transversal parametric spring spaces.

Theorem 29. (Characterization of the common fixed point). Let $f$ and $h$ be maps from a transversal upper parametric spring space $X:=(X, M)$ into itself which is with the property of HCS-convergence, where $t \mapsto M(u, v, t)$ is decreasing and $(u, v) \mapsto M(u, v, t)$ is continuous with respect to sequences and symmetrical.

Then $f$ and $h$ have a common fixed point in $X$ if and only if there exists a continuous with respect to sequences mapping $T: X \rightarrow f(X) \cap h(X)$ such that the pair $\{f, T\}$ is compatible and the pair $\{h, T\}$ is weakly compatible and

$$
M(T x, T y, \varphi(t)) \preccurlyeq M(f x, h y, t) \quad \text { for all } \quad x, y \in X,
$$

where $\varphi: \mathbb{S} \rightarrow \mathbb{S}$ is a function satisfying $c \prec \varphi(t) \prec t$ for every $t \succ c \succcurlyeq a$. Also, in this case, $f, h$ and $T$ have a unique common fixed point in $X$.

Proof. Necessity. Suppose $f(\xi)=h(\xi)=\xi$ for some $\xi \in X$ and let $T(x)=\xi$ for all $x \in X$. Thus, $T$ is a continuous mapping of $X$ into $f(X) \cap h(X)$. Also, $T$ commutes with $f$ and $h$ and thus $\{f, T\}$ is compatible and $\{h, T\}$ is weakly compatible. On the other hand, for any $t \succ c(t \in \mathbb{S})$, 
we obtain

$$
M(T x, T y, \varphi(t))=M(\xi, \xi, \varphi(t))=a \preccurlyeq M(f x, h y, t)
$$

for all $x, y \in X$. This proves the necessity.

Sufficiency. Conversely of the preceding conditions, suppose that $T$ is with the properties in statement. Define $y_{0}=f\left(x_{0}\right)$ for an arbitrary fixed $x_{0} \in X$. Since $T(X) \subset h(X)$ we can choose a point $x_{1} \in X$ such that $h\left(x_{1}\right)=T\left(x_{0}\right)=y_{1}$. Inequality $T(X) \subset f(X)$ yields a point $x_{2} \in X$ such that $f\left(x_{2}\right)=T\left(x_{1}\right)=y_{2}$. In further, having chosen the point $x_{2 n-2}$ we choose a point $x_{2 n-1}$ such that $h\left(x_{2 n-1}\right)=T\left(x_{2 n-2}\right)=y_{2 n-1}$. Also, for the point $x_{2 n}$ we have $f\left(x_{2 n}\right)=T\left(x_{2 n-1}\right)=y_{2 n}$. From (M) we have the following inequality in the form

$$
\begin{gathered}
M\left(y_{2 n+1}, y_{2 n+2}, \varphi(t)\right)=M\left(T\left(x_{2 n}\right), T\left(x_{2 n+1}\right), \varphi(t)\right) \preccurlyeq \\
\preccurlyeq M\left(f\left(x_{2 n}\right), h\left(x_{2 n+1}\right), t\right)=M\left(y_{2 n}, y_{2 n+1}, t\right),
\end{gathered}
$$

as and the following inequality, similarly in the following form via symmetry as

$$
\begin{aligned}
& M\left(y_{2 n+2}, y_{2 n+3}, \varphi(t)\right)=M\left(T\left(x_{2 n+2}\right), T\left(x_{2 n+1}\right), \varphi(t)\right) \preccurlyeq \\
& \preccurlyeq M\left(f\left(x_{2 n+2}\right), h\left(x_{2 n+1}\right), t\right)=M\left(y_{2 n+1}, y_{2 n+2}, t\right),
\end{aligned}
$$

which means that the following inequality holds in the form as

$$
M\left(y_{n}, y_{n+1}, \varphi(t)\right) \preccurlyeq M\left(y_{n-1}, y_{n}, t\right) \quad \text { for every } n \in \mathbb{N} .
$$

Hence, the sequence $\left\{M\left(y_{n}, y_{n+1}, t\right)\right\}_{n \in \mathbb{N}}$ is a convergent sequence in $[a, b]$. This implies (from HCS-convergence) that its sequence $\left\{y_{n}\right\}_{n \in \mathbb{N}}$ contains convergent subsequences $\left\{y_{n(k)}\right\}_{k \in \mathbb{N}}$ with limit $\xi \in X$, such that hence we obtain

$$
\lim _{k \rightarrow \infty} y_{n(k)}=\lim _{k \rightarrow \infty} f\left(x_{2 n(k)}\right)=\lim _{k \rightarrow \infty} h\left(x_{2 n(k)-1}\right)=\xi .
$$

Since $T$ is continuous and the pair $\{f, T\}$ is compatible, we have $T(\xi)=$ $\lim _{k \rightarrow \infty} f T\left(x_{2 n(k)}\right)$. Now for any $t \succ c(t \in \mathbb{S})$ we obtain

$$
\begin{gathered}
M\left(T\left(T x_{2 n(k)}\right), T\left(x_{2 n(k)-1}\right), \varphi^{m+1}(t)\right) \preccurlyeq \\
\preccurlyeq M\left(f\left(T x_{2 n(k)}\right), h\left(x_{2 n(k)-1}\right), \varphi^{m}(t)\right)
\end{gathered}
$$

for all $m \in \mathbb{N} \cup\{0\}$. Thus, as $k \rightarrow \infty$, we have the following inequality in the form as

$$
M\left(T(\xi), \xi, \varphi^{m+1}(t)\right) \preccurlyeq M\left(T(\xi), \xi, \varphi^{m}(t)\right),
$$


for all $m \in \mathbb{N} \cup\{0\}$, i.e., we obtain and the following inequality in the form as

$$
M\left(T(\xi), \xi, \varphi^{m+1}(t)\right) \preccurlyeq M(T(\xi), \xi, t)
$$

for all $m \in \mathbb{S} \cup\{0\}$. Since $t \mapsto M(u, v, t)$ is a decreasing function and $\varphi(t) \prec t$ for every $t \in \mathbb{S}$ we obtain the following inequality in the form as

$$
M(T(\xi), \xi, t) \preccurlyeq M\left(T(\xi), \xi, \varphi^{m+1}(t)\right),
$$

such that from (39) and (40) we obtain the following equality

$$
M\left(T(\xi), \xi, \varphi^{m+1}(t)\right)=M(T(\xi), \xi, t)=\text { Constant } \in[a, b] .
$$

Taking $t \rightarrow \sup \mathbb{S}$ we obtain that Constant $=a$ and hence $T(\xi)=\xi$ for some $\xi \in X$. On the other hand, since $T(X) \subset f(X)$ there exists $z \in X$ such that $f(z)=T(\xi)$. If $T(z)=\xi$, from $(\mathrm{M})$, we obtain

$$
M\left(T(z), T\left(x_{2 n(k)-1}\right), \varphi(t)\right) \preccurlyeq M\left(f(z), h\left(x_{2 n(k)-1}\right), t\right)
$$

hence, as $k \rightarrow \infty$ to obtain for any $t \succ c(t \in \mathbb{S})$ that

$$
M(T(z), \xi, \varphi(t)) \preccurlyeq M(f(z), \xi, t)=M(\xi, \xi, t)=a,
$$

however, on the other hand, since $M(T(z), \xi, t) \preccurlyeq M(T(z), \xi, \varphi(t))$ it follows $M(T(z), \xi, t)=a$ for every $t \succ c(t \in \mathbb{S})$; which means $T(z)=\xi=$ $T(\xi)=f(z)$. Now $\{f, T\}$ is compatible, $T(z)=f(z)$ implies that $T(f(z))=$ $f(T(z))$, i.e., hence $f(\xi)=\xi$. Also, for $T(X) \subset h(X)$ we obtain that there exists an $r \in X$ such that $T(\xi)=h(r)$. We show that $T(r)=\xi$. Applying (M) for any $t \succ c(t \in \mathbb{S})$ we have

$$
M(T(\xi), T(r), \varphi(t)) \preccurlyeq M(f(\xi), h(r), t)=M(\xi, \xi, t)=a,
$$

and so $M(T(\xi), T(r), t) \preccurlyeq M(T(\xi), T(r), \varphi(t)) \preccurlyeq a$, i.e., thus $T(r)=T(\xi)$. Since $\{h, T\}$ is weakly compatible, $T(r)=h(r)$ implies that $h(T(r))=$ $T(h(r))$, and thus $h(\xi)=h(T(r))=T(h(r))=T(\xi)=\xi$, which means that $\xi$ is a common fixed point of three mappings $f, h$ and $T$ which is sufficiency.

In further, suppose that $u$ and $v$ are two different common fixed points of three maps $f, h$ and $T$. Then, from (M), we obtain

$$
\begin{aligned}
& M\left(u, v, \varphi^{m+1}(t)\right)=M\left(T(u), T(v), \varphi^{m+1}(t)\right) \preccurlyeq \\
& \preccurlyeq M\left(f(u), h(v), \varphi^{m}(t)\right)=M\left(u, v, \varphi^{m}(t)\right),
\end{aligned}
$$

hence $M\left(u, v, \varphi^{m+1}(t)\right) \preccurlyeq M(u, v, t)$. Because, for any $t \succ c(t \in \mathbb{S})$ we have $M(u, v, t) \preccurlyeq M\left(u, v, \varphi^{m+1}(t)\right)$, which means that $M(u, v, t)=$ $M\left(u, v, \varphi^{m+1}(t)\right)=$ Constant $\in[a, b]$. Hence, as $t \rightarrow \sup \mathbb{S}$, we obtain that Constant $=a$, i.e., $u=v$. The proof is complete.

Consequenceses of Theorem 29. As immediate applications of Theorem 29 first we have many examples different spaces for which Theorem 
29 holds. Also, second, as immediate consequences of Theorem 29 we can obtain special statements via special conditions (necessary and sufficient) for existing a unique common fixed point for four mappings.

We notice that the lower transversal parametric spaces have been introduced in 1998 by Tasković [37]. For further facts on lower transversal parametric spring spaces see: Tasković [51].

Let $X$ be a nonempty set, let $P:=(P, \preccurlyeq)$ be a partially ordered set, and let $\mathbb{S}$ be a totally ordered set. In connection with the preceding facts, the function $N: X \times X \times \mathbb{S} \rightarrow[a, b]$ (or $N: X \times X \times \mathbb{S} \rightarrow(a, b]$ ) for $a \prec b$ is called a lower parametric spring transverse on $X$ (or lower parametric spring transversal) iff: there is $c \in \mathbb{S}$ such that $N(u, v, t)=b$ for every $t \succ c \succcurlyeq a$ if and only if $u=v$, and $N(u, v, t) \rightarrow b$ as $t \rightarrow \sup \mathbb{S}($ or $N(u, v, t)=N(u, v, s)$ and $t \neq s$ implies $u=v$ ) for all $u, v \in X$.

A lower transversal parametric spring space is a set $X$ together with a given lower parametric spring transverse $N: X \times X \times \mathbb{S} \rightarrow[a, b]$ for $a \prec b$, in notation $X:=(X, N)$. The element $b \in(a, b]$ is called spring of space $X$.

Otherwise, the function $N$ is called a semilower parametric spring transverse on $X$ (or semilower parametric spring transversal) iff: there is $c \in \mathbb{S}$ such that $N(u, v, t)=b$ for every $t \succ c \succcurlyeq a$ implies $u=v$, and $N(u, v, t) \rightarrow b$ as $t \rightarrow \sup \mathbb{S}$ (or $N(u, v, t)=N(u, v, s)$ and $t \neq s$ implies $u=v$ ) for all $u, v \in X$. A semilower transversal parametric spring space $X:=(X, N)$ is a set $X$ together with a given semilower parametric spring transverse on $X$.

Let $X:=(X, N)$ be a lower transversal parametric spring space. For $S \subset X$ we denoted tpc. $\operatorname{diam}(S)$ as a transversal parametric spring diameter of $S$, in the sense that

$$
\operatorname{tpc} \operatorname{diam}(S):=\inf \{N(x, y, t): x, y \in S\},
$$

where $t \in \mathbb{S}$ and $S \subset Y$ implies tpc. $\operatorname{diam}(S) \succcurlyeq \operatorname{tpc} \operatorname{diam}(Y)$.

Elements of a lower parametric spring transversal space will usually be called points. Given a lower parametric spring transversal space $X:=(X, N)$ and a point $z \in X$, the open ball of center $z$ and radius $r$ is the set

$$
N(B(z, r)):=\{x \in X: N(z, x, t) \succ r\}
$$

where $t \in \mathbb{S}$. The lower parametric spring convergence $x_{n} \rightarrow x$ as $n \rightarrow \infty$ in the lower transversal parametric spring space $X:=(X, N)$ means that the following fact holds that

$$
N\left(x_{n}, x, t\right) \rightarrow b \quad(\text { for } t \in \mathbb{S}) \quad \text { as } \quad n \rightarrow \infty .
$$

The sequence $\left\{x_{n}\right\}_{n \in \mathbb{N}}$ in the lower transversal parametric spring space $X:=(X, N)$ is called lower transversal parametric spring sequence 
(or lower parametric spring Cauchy sequence) iff: for every increasing sequence $\left\{b_{n}\right\}_{n \in \mathbb{N}}$ which converge to $b$ there is an $n_{0}=n_{0}(\varepsilon)$ such that

$$
N\left(x_{n}, x_{m}, t\right) \succ b_{n} \quad \text { for all } n, m \geq n_{0}
$$

and $t \in \mathbb{S}$. Let $X$ be a lower transversal parametric spring space. We notice, from Tasković [54], that a sequence $\left\{x_{n}\right\}_{n \in \mathbb{N}}$ in $X$ is said to be lower transversal parametric spring sequence if and only if

$$
\lim _{n \rightarrow \infty}\left(\operatorname{tpc} \cdot \operatorname{diam}\left\{x_{k}: k \geq n\right\}\right)=b .
$$

In this sense, a lower transversal parametric spring space is called lower parametric spring complete iff every lower transversal parametric spring sequence lower parametric spring converges. Also, a space $X:=(X, N)$ is said to be lower parametric spring orbitally complete (or lower parametric spring T-orbitally complete) iff every lower parametric spring transversal sequence which contained in $\mathcal{O}(x):=\left\{x, T x, T^{2} x, \ldots\right\}$ for some $x \in X$ lower parametric spring converges in $X$.

For the convergence of a sequence $\left\{x_{n}\right\}_{n \in \mathbb{N}}$ in the lower transversal parametric spring spaces see: Tasković [54, p. 87, 88 and 89].

Let $X$ be a lower transversal parametric spring space. We shall considered the concept of HCS-convergence in a space $X$ by Tasković [51]; i.e., a lower transversal parametric spring space $X$ satisfies the condition of HCSconvergence iff $\left\{x_{n}\right\}_{n \in \mathbb{N}}$ is a sequence in $X$ and if the convergence of the sequence $\left\{M\left(x_{n}, x_{n+1}, t\right)\right\}_{n \in \mathbb{N}}$ implies that $\left\{x_{n}\right\}_{n \in \mathbb{N}}$ has two convergent subsequences $\left\{x_{2 n(r)}\right\}_{r \in \mathbb{N}}$ and $\left\{x_{2 n(r)+1}\right\}_{r \in \mathbb{N}}$ to a point $\xi \in X$. For this form of convergence on topological spaces see: Tasković [37, p. 61]. For an another convergence (in the classical sense) on the lower transversal parametric spaces see: Tasković [50].

Also, let $f$ and $h$ be self maps on a transversal lower parametric spring space $X$. They are compatible if $\lim _{n \rightarrow \infty} f h\left(x_{n}\right)=\lim _{n \rightarrow \infty} h f\left(x_{n}\right)$, whenever $\left\{x_{n}\right\}_{n \in \mathbb{N}}$ is a sequence in $X$ such that $\lim _{n \rightarrow \infty} f\left(x_{n}\right)=\lim _{n \rightarrow \infty} h\left(x_{n}\right)=$ $\xi$ for some point $\xi \in X$.

On the other hand, the mappings $f$ and $h$ from a transversal lower parametric spring space $X$ into itself are weakly compatible if they commute at their coincidence point, i.e., $f(\xi)=h(\xi)$ implies that $f h(\xi)=h f(\xi)$. We notice that a pair $\{f, h\}$ of compatible maps is weakly compatible, but converse is not true in general.

We are now in a position to formulate our following statement, as a consequence of the preceding facts, on lower transversal parametric spring spaces.

Theorem 30. (Characterization of the common fixed point). Let $f$ and $h$ be maps from a transversal lower parametric spring space $X:=(X, N)$ into itself which is with the property of HCS-convergence, where $t \mapsto N(u, v, t)$ is increasing and $(u, v) \mapsto N(u, v, t)$ is continuous with respect to sequences and symmetrical. 
Then $f$ and $h$ have a common fixed point in $X$ if and only if there exists a continuous with respect to sequences mapping $T: X \rightarrow f(X) \cap h(X)$ such that the pair $\{f, T\}$ is compatible and the pair $\{h, T\}$ is weakly compatible and

$$
N(T x, T y, \varphi(t)) \succcurlyeq N(f x, h y, t) \quad \text { for all } \quad x, y \in X,
$$

where $\varphi: \mathbb{S} \rightarrow \mathbb{S}$ is a strictly increasing function for any $t \succ c \succcurlyeq$ a satisfying $\varphi(c)=c$ and $\varphi(t) \prec t$ for any $t \succ c \succcurlyeq a$. Also, in this case, $f, h$ and $T$ have a unique common fixed point in $X$.

Proof. Necessity. Suppose $f(\xi)=h(\xi)=\xi$ for some $\xi \in X$ and let $T(x)=\xi$ for all $x \in X$. Thus, $T$ is a continuous mapping of $X$ into $f(X) \cap h(X)$. Also, $T$ commutes with $f$ and $h$ and thus $\{f, T\}$ is compatible and $\{h, T\}$ is weakly compatible. On the other hand, for any $t \succ c(t \in \mathbb{S})$, since $\varphi(t) \succ \varphi(c)=c$, we obtain

$$
N(T x, T y, \varphi(t))=N(\xi, \xi, \varphi(t))=b \succcurlyeq N(f x, h y, t)
$$

for all $x, y \in X$. This proves the necessity.

Sufficiency. Conversely of the preceding conditions, suppose that $T$ is with the properties in statement. Define $y_{0}=f\left(x_{0}\right)$ for an arbitrary fixed $x_{0} \in X$. Since $T(X) \subset h(X)$ we can choose a point $x_{1} \in X$ such that $h\left(x_{1}\right)=T\left(x_{0}\right)=y_{1}$. Inequality $T(X) \subset f(X)$ gives a point $x_{2} \in X$ such that $f\left(x_{2}\right)=T\left(x_{1}\right)=y_{2}$. In further, having chosen the point $x_{2 n-2}$ we choose a point $x_{2 n-1}$ such that $h\left(x_{2 n-1}\right)=T\left(x_{2 n-2}\right)=y_{2 n-1}$. Also, for the point $x_{2 n}$ we have $f\left(x_{2 n}\right)=T\left(x_{2 n-1}\right)=y_{2 n}$. From $(\mathrm{N})$ we have the following inequality in the form

$$
\begin{gathered}
N\left(y_{2 n+1}, y_{2 n+2}, \varphi(t)\right)=N\left(T\left(x_{2 n}\right), T\left(x_{2 n+1}\right), \varphi(t)\right) \succcurlyeq \\
\succcurlyeq N\left(f\left(x_{2 n}\right), h\left(x_{2 n+1}\right), t\right)=N\left(y_{2 n}, y_{2 n+1}, t\right),
\end{gathered}
$$

as and the following inequality, similarly in the following form via symmetry as

$$
\begin{gathered}
N\left(y_{2 n+2}, y_{2 n+3}, \varphi(t)\right)=N\left(T\left(x_{2 n+2}\right), T\left(x_{2 n+1}\right), \varphi(t)\right) \succcurlyeq \\
\succcurlyeq N\left(f\left(x_{2 n+2}\right), h\left(x_{2 n+1}\right), t\right)=N\left(y_{2 n+1}, y_{2 n+2}, t\right),
\end{gathered}
$$

which means that the following inequality holds in the form as

$$
N\left(y_{n}, y_{n+1}, \varphi(t)\right) \succcurlyeq N\left(y_{n-1}, y_{n}, t\right) \quad \text { for every } n \in \mathbb{N} \text {. }
$$

Hence, the sequence $\left\{N\left(y_{n}, y_{n+1}, t\right)\right\}_{n \in \mathbb{N}}$ is a convergent sequence in $[a, b]$. This implies (from HCS-convergence) that its sequences $\left\{y_{n}\right\}_{n \in \mathbb{N}}$ contains convergent subsequences $\left\{y_{n(k)}\right\}_{k \in \mathbb{N}}$ with limit $\xi \in X$, such that hence we obtain

$$
\lim _{k \rightarrow \infty} y_{n(k)}=\lim _{k \rightarrow \infty} f\left(x_{2 n(k)}\right)=\lim _{k \rightarrow \infty} h\left(x_{2 n(k)-1}\right)=\xi .
$$


Since $T$ is continuous and the pair $\{f, T\}$ is compatible, we have $T(\xi)=$ $\lim _{k \rightarrow \infty} f T\left(x_{2 n(k)}\right)$. Now for $t \succ c(t \in \mathbb{S})$ we obtain

$$
\begin{aligned}
& N\left(T\left(T x_{2 n(k)}\right), T\left(x_{2 n(k)-1}\right), \varphi^{m+1}(t)\right) \succcurlyeq \\
& \succcurlyeq N\left(f\left(T x_{2 n(k)}\right), h\left(x_{2 n(k)-1}\right), \varphi^{m}(t)\right)
\end{aligned}
$$

for all $m \in \mathbb{N} \cup\{0\}$. Thus, as $k \rightarrow \infty$, we have the following inequality in the form as

$$
N\left(T(\xi), \xi, \varphi^{m+1}(t)\right) \succcurlyeq N\left(T(\xi), \xi, \varphi^{m}(t)\right),
$$

for all $m \in \mathbb{N} \cup\{0\}$, i.e., we obtain and the following inequality in the form as

$$
N\left(T(\xi), \xi, \varphi^{m+1}(t)\right) \succcurlyeq N(T(\xi), \xi, t)
$$

for all $m \in \mathbb{N} \cup\{0\}$. Since $t \mapsto N(u, v, t)$ is an increasing function and $\varphi(t) \prec t$ for every $t \in \mathbb{S}$ we obtain the following inequality in the form as

$$
N(T(\xi), \xi, t) \succcurlyeq N\left(T(\xi), \xi, \varphi^{m+1}(t)\right),
$$

such that from (41) and (42) we obtain the following equality

$$
N\left(T(\xi), \xi, \varphi^{m+1}(t)\right)=N(T(\xi), \xi, t)=\text { Constant } \in[a, b] .
$$

Taking $t \rightarrow \sup \mathbb{S}$ we obtain that Constant $=b$ and hence $T(\xi)=\xi$ for some $\xi \in X$. On the other hand, since $T(X) \subset f(X)$ there exists $z \in X$ such that $f(z)=T(\xi)$. If $T(z)=\xi$, from $(\mathrm{N})$, we obtain

$$
N\left(T(z), T\left(x_{2 n(k)-1}\right), \varphi(t)\right) \succcurlyeq N\left(f(z), h\left(x_{2 n(k)-1}\right), t\right)
$$

hence, as $k \rightarrow \infty$ to obtain for $t \succ c(t \in \mathbb{S})$ that

$$
N(T(z), \xi, \varphi(t)) \succcurlyeq N(f(z), \xi, t)=N(\xi, \xi, t)=b,
$$

however, on the other hand, since $N(T(z), \xi, t) \succcurlyeq N(T(z), \xi, \varphi(t))$ it follows $N(T(z), \xi, t)=b$ for every $t \succ c(t \in \mathbb{S})$; which means $T(z)=$ $\xi=T(\xi)=f(z)$. Now $\{f, T\}$ is compatible, $T(z)=f(z)$ implies that $T(f(z))=f(T(z))$, i.e., hence $f(\xi)=\xi$. Also, for $T(X) \subset h(X)$ we obtain that there exists an $r \in X$ such that $T(\xi)=h(r)$. We show that $T(r)=\xi$. Applying $(\mathrm{N})$ for $t \succ c(t \in \mathbb{S})$ we have

$$
N(T(\xi), T(r), \varphi(t)) \succcurlyeq N(f(\xi), h(r), t)=N(\xi, \xi, t)=b,
$$

and so $N(T(\xi), T(r), t) \succcurlyeq N(T(\xi), T(r), \varphi(t)) \succcurlyeq b$, i.e., thus $T(r)=T(\xi)$. Since $\{h, T\}$ is weakly compatible, $T(r)=h(r)$ implies that $h(T(r))=$ $T(h(r))$, and thus $h(\xi)=h(T(r))=T(h(r))=T(\xi)=\xi$, which means that $\xi$ is a common fixed point of three mappings $f, h$ and $T$ which is sufficiency. 
In further, suppose that $u$ and $v$ are two different common fixed points of three maps $f, h$ and $T$. Then, from $(\mathrm{N})$, we obtain

$$
\begin{gathered}
N\left(u, v, \varphi^{m+1}(t)\right)=N\left(T(u), T(v), \varphi^{m+1}(t)\right) \succcurlyeq \\
\succcurlyeq M\left(f(u), h(v), \varphi^{m}(t)\right)=N\left(u, v, \varphi^{m}(t)\right),
\end{gathered}
$$

hence $N\left(u, v, \varphi^{m+1}(t)\right) \succcurlyeq N(u, v, t)$. Because, for $t \succ c(t \in \mathbb{S})$ we have $N(u, v, t) \succcurlyeq N\left(u, v, \varphi^{m+1}(t)\right)$, which means that

$$
N(u, v, t)=N\left(u, v, \varphi^{m+1}(t)\right)=\text { Constant } \in[a, b] .
$$

Hence, as $t \rightarrow \sup \mathbb{S}$, we obtain that Constant $=b$, i.e., $u=v$. The proof is complete.

Consequenceses of Theorem 30. As immediate applications of Theorem 30 first we have many examples different spaces for which Theorem 30 holds. Also, second, as immediate consequences of Theorem 30 we can obtain special statements via special conditions (necessary and sufficient) for existing a unique common fixed point for four mappings.

Middle transversal parametric spring spaces. We notice that the middle transversal parametric spaces have been introduced in mathematics in the year 1998 by Tasković with only other name as transversal spaces. This spaces are extension so-called middle transversal interval spaces which was introduced in 2003 by Tasković [46]. For further facts on middle transversal parametric spaces see: Tasković [51].

In the preceding context, a middle transversal parametric spring space (or middle parametric spring space) is an upper transversal parametric spring space and a lower transversal parametric spring space, simultaneous.

In other vords, a middle transversal parametric spring space in notation $X:=(X, M, N)$ is a set $X$ together with a given upper parametric spring transverse $M: X \times X \times \mathbb{S} \rightarrow[a, b] \subset P$ and with a given lower parametric spring transverse $N: X \times X \times \mathbb{S} \rightarrow[a, b] \subset P$ for $a \prec b$. We notice that a middle transversal parametric spring space has two springs: $a$ and $b$.

Elements of a middle transversal parametric spring space will usually be called points. Given a middle transversal parametric spring space $X:=$ $(X, M, N)$ and a point $z \in X$, the open ball of center $z$ and radius $r$ is the set defined for $t \in \mathbb{S}$ as

$$
H(B(z, r, t)):=\{x \in X: N(z, x, t) \succ r, M(z, x, t) \prec r\} .
$$

The convergence $x_{n} \rightarrow x$ as $n \rightarrow \infty$ in the middle transversal parametric spring space $X:=(X, M, N)$ means that

$$
M\left(x_{n}, x, t\right) \rightarrow a \text { and } N\left(x_{n}, x, t\right) \rightarrow b \quad \text { as } n \rightarrow \infty
$$


for arbitrary $t \in \mathbb{S}$. The sequence $\left\{x_{n}\right\}_{n \in \mathbb{N}}$ in the middle transversal parametric spring space $X:=(X, M, N)$ is called middle transversal parametric spring sequence (or middle parametric spring Cauchy sequence) iff for every decreasing sequence $\left\{a_{n}\right\}_{n \in \mathbb{N}}$ which converge to $a$ and for every increasing sequence $\left\{b_{n}\right\}_{n \in \mathbb{N}}$ which converge to $b$ and for every $t \in \mathbb{S}$ there is an $n_{0}=n_{0}(\varepsilon, t)$ such that

$$
M\left(x_{n}, x_{m}, t\right) \prec a_{n} \quad \text { and } \quad N\left(x_{n}, x_{m}, t\right) \succ b_{n}
$$

for all $n, m \geq n_{0}$.

In this sense, a middle transversal parametric spring space is called middle complete iff every middle transversal parametric spring sequence converges.

Also, a space $X:=(X, M, N)$ is said to be middle parametric spring orbitally complete (or middle parametric spring T-orbitally complete) iff every middle parametric spring transversal sequence which contained in the orbit $\mathcal{O}(x)$ for some $x \in X$ converges in $X$.

We are now in a position to formulate our the following statement, as a consequence of the preceding results, on middle transversal parametric spring spaces.

Theorem 31. Let $f, h$ and $\mu$ be maps from a transversal middle parametric spring space $X:=(X, M, N)$ into itself, where $c=a$ and where $X$ satisfies the condition of HCS-convergence. Suppose that $t \mapsto M(u, v, t)$ is decreasing, $t \mapsto N(u, v, t)$ is increasing, $(u, v) \mapsto M(u, v, t)$ and $(u, v) \mapsto N(u, v, t)$ are continuous with respect to sequences and symmetrical.

Then $f, h$ and $\mu$ have a common fixed point in $X$ if and only if there exists a continuous with respect to sequences mapping $T: X \rightarrow f(X) \cap h(X) \cap \mu(X)$ such the pair $\{f, T\}$ is compatible, the pair $\{h, T\}$ is weakly compatible and the pair $\{\mu, T\}$ is compatible, and

$$
M(T x, T y, \varphi(t)) \preccurlyeq M(f x, h y, t)
$$

and

$$
N(T x, T y, \varphi(t)) \succcurlyeq N(\mu x, h y, t)
$$

for all $x, y \in X$, where $\varphi: \mathbb{S} \rightarrow \mathbb{S}(\varphi(c)=c)$ is a strictly increasing function for every $t \succ c \succcurlyeq a$ satisfying $\varphi(t) \prec t$ for every $t \succ c \succcurlyeq a$. Also, in this case, $f, h, \mu$ and $T$ have a unique common fixed point in $X$.

Proof. Applying Theorem 29 we obtain that $T(\xi)=f(\xi)=h(\xi)=\xi$ for some $\xi \in X$. On the other hand, applying Theorem 30, it follows that $T(\eta)=\mu(\eta)=h(\eta)=\eta$ for some $\eta \in X$. We complete the proof by showing that $f, h, \mu$ and $T$ can have at most one common fixed point: for, if $\xi \neq \eta$ 
were two common fixed points (in the preceding sense), then we have

$$
\begin{aligned}
& M(\xi, \eta, \varphi(t))=M(T(\xi), T(\eta), \varphi(t)) \preccurlyeq M(f(\xi), h(\eta), t)=M(\xi, \eta, t), \\
& N(\xi, \eta, \varphi(t))=N(T(\xi), T(\eta), \varphi(t)) \succcurlyeq N(\mu(\xi), h(\eta), t)=N(\xi, \eta, t),
\end{aligned}
$$

i.e., $M\left(\xi, \eta, \varphi^{n}(t)\right) \preccurlyeq M(\xi, \eta, t)$ and $N\left(\xi, \eta, \varphi^{n}(t)\right) \succcurlyeq N(\xi, \eta, t)$, hence applying the preceding facts we obtain $M(\xi, \eta, a)=b \preccurlyeq M(\xi, \eta, t)$ and $N(\xi, \eta, a)=$ $a \succcurlyeq N(\xi, \eta, t)$, which is contradicting, i.e., $\xi=\eta$. Also, this means uniqueness. The proof is complete.

\section{Forked Points on Topological Spaces}

Let $X$ be an arbitrary nonempty set, $T$ be a mapping from $X$ into $X$, and $P:=(P, \preccurlyeq)$ a nonempty partially ordered set. A mapping $f: X \rightarrow P$ (or $T: X \rightarrow X$ ) has a forked point (or furcate point) $p \in X$ if the following equality holds in the form

$$
f(p)=f(T p) \text { for some } p \in X ;
$$

frequently, we say that in this case (Ra), the mapping $f: X \rightarrow P$ has a pair $(p, T p)$ of bifurcation points, or that $T: X \rightarrow X$ has a forks point $p \in X$.

In this context, we notice that the preceding statements, de facto, are results on forked points. If $X=P=[0,1]$, then an illustrative situation of the forked points is given on Figs. 5, 6 and 7 .

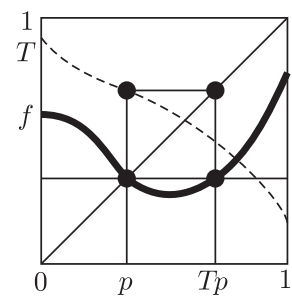

Figure 5

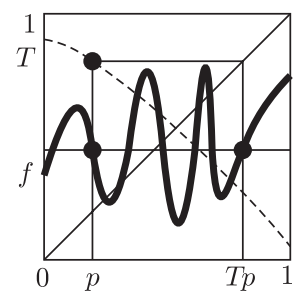

FiguRE 6

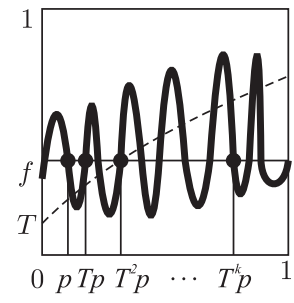

FIGURE 7 
We notice that many problems in nonlinear functional analysis (as and in the fixed point theory) are reducible to the existence of forked points of certain mappings.

Further, let $P:=(P, \preccurlyeq)$ be a partially ordered set with a minimum (or with the property that every nonempty subset in $P$ has an infimum) such that every decreasing sequence $\left\{x_{n}\right\}_{n \in \mathbb{N}}$ in $P$ has a limit in $P$, denoted by $\lim _{n \rightarrow \infty} x_{n}$.

In connection with this, we shall introduce the concept of lower ordered RBS-convergence in a topological space $X$ for $B: X \rightarrow P$, i.e., a topological space $X$ satisfies the condition of lower ordered RBS-convergence iff $\left\{a_{n}(x)\right\}_{n \in \mathbb{N}}$ is an arbitrary sequence in $X$ with arbitrary $x \in X$ and if $B\left(a_{n}(x)\right) \rightarrow b=b(x) \in P(n \rightarrow \infty)$ implies that $\left\{a_{n}(x)\right\}_{n \in \mathbb{N}}$ has a convergent subsequence $\left\{a_{n(k)}(x)\right\}_{k \in \mathbb{N}}$ which converges to $\xi \in X$, where

$$
B(\xi) \preccurlyeq \inf _{x \in X} \lim _{k \rightarrow \infty} B\left(a_{n(k)}(x)\right) .
$$

Theorem 32. (Forked points existence, Tasković [51]). Let $T$ be a mapping of a topological space $X$ into itself, where $X$ satisfies the condition of lower ordered RBS-convergence. If

$$
B(T x) \preccurlyeq B(x) \quad \text { for every } \quad x \in X,
$$

then for $T$ there exists a forked point $\xi \in X$, i.e., then the following equalities hold in the form

$$
B(T \xi)=B(\xi)=\alpha:=\inf _{x \in X} \lim _{n \rightarrow \infty} B\left(b_{n}(x)\right)
$$

for some sequence $\left\{b_{n}(x)\right\}_{n \in \mathbb{N}}$ in $X$ which converges to the forked point $\xi \in X$.

A brief proof of this statement based on some elementary facts may be found in Taskovi ć [51]. For this, also see Taskovi ć [40].

Proof of Theorem 32. Let $x$ be an arbitrary point in $X$. Then from the inequality $(\mathrm{Bu})$ we obtain the following inequalities in the form

$$
\cdots \preccurlyeq B\left(T^{n+1} x\right) \preccurlyeq B\left(T^{n} x\right) \preccurlyeq \cdots \preccurlyeq B(T x) \preccurlyeq B(x)
$$

for every $n \in \mathbb{N} \cup\{0\}$ and for every $x \in X$. Thus, for the sequence $\left\{B\left(T^{n} x\right)\right\}_{n \in \mathbb{N} \cup\{0\}}$ from (45), we obtain that $B\left(T^{n} x\right) \rightarrow b \in P(n \rightarrow \infty)$ with arbitrary $x \in X$. This implies (from the lower ordered RBS-convergence) that its sequence $\left\{T^{n} x\right\}_{n \in \mathbb{N} \cup\{0\}}$ contains a convergent subsequence $\left\{T^{n(k)}(x)\right\}_{k \in \mathbb{N}}$ with a limit point $\xi \in X$. Since $X$ satisfies the condition of lower ordered RBS-convergence, from (45), we have

$$
\begin{gathered}
\alpha:=\inf _{x \in X} \lim _{n \rightarrow \infty} B\left(T^{n} x\right) \preccurlyeq \lim _{n \rightarrow \infty} B\left(T^{n} \xi\right) \preccurlyeq \cdots \\
\cdots \preccurlyeq B(T \xi) \preccurlyeq B(\xi) \preccurlyeq \inf _{x \in X} \lim _{k \rightarrow \infty} B\left(T^{n(k)} x\right)=\alpha ;
\end{gathered}
$$


i.e., $B(T \xi)=B(\xi)=\alpha$. This means that (Ri) holds, i.e., that the mapping $B: X \rightarrow P$ has a forked point $\xi \in X$, where the existing sequence $\left\{b_{n}(x)\right\}_{n \in \mathbb{N}}$, de facto, is the preceding subsequence of the sequence of iterates $\left\{T^{n(k)} x\right\}_{k \in \mathbb{N}}$. The proof is complete.

Let $X$ be an arbitrary nonempty set, $T: X \rightarrow X$, and $P:=(P, \preccurlyeq)$ be a nonempty poset. A mapping $f: X \rightarrow P$ (or $T: X \rightarrow X$ ) has a $k$-forked point (or $k$-furcate point) $p \in X$ if for arbitrary fixed integer $k \geqslant 1$ the following equalities hold in the form

$$
f\left(T^{k} p\right)=\cdots=f(T p)=f(p) \text { for some } p \in X ;
$$

Frequently, we say that in this case (Rk), the mapping $f: X \rightarrow P$ has cycle or $k$-pair $\left(p, T p, \ldots, T^{k} p\right)$ of bifurcation points, or that $T: X \rightarrow X$ has a $k$-forks point.

In connection with this, from the proof of Theorem 32, we obtain, as a direct extension of the preceding result, the following general statement.

Theorem 33. (Existence of $k$-forked points). Let $T$ be a mapping of a topological space $X$ into itself, where $X$ satisfies the condition of lower ordered $R B S$-convergence. If

$$
B(T x) \preccurlyeq B(x) \quad \text { for every } \quad x \in X,
$$

then for $T$ there exists an $k$-forked point $\xi \in X$, i.e., then the following equalities hold in the form

$$
B\left(T^{k} \xi\right)=\cdots=B(T \xi)=B(\xi)=\alpha:=\inf _{x \in X} \lim _{n \rightarrow \infty} B\left(b_{n}(x)\right)
$$

for an arbitrary fixed integer $k \geqslant 1$ and for some sequence $\left\{b_{n}(x)\right\}_{n \in \mathbb{N}}$ in $X$ which converges to $\xi \in X$.

Adequately with the preceding, let $X$ be an arbitrary nonempty set, $T$ : $X \rightarrow X$, and $P:=(P, \preccurlyeq)$ be a nonempty poset. A mapping $f: X \rightarrow P$ (or $T: X \rightarrow X$ ) has a $m(k)$-forked point (or $m(k)$-furcate point) $p \in X$ if (Rm) $f\left(T^{m} p\right)=\cdots=f\left(T^{k} p\right)=\cdots=f(T p)=f(p)$ for some $p \in X$,

for a fixed integer $k \geqslant 1$ and for an arbitrary integer $m \geqslant k$. In this case, we say frequently that the mapping $f: X \rightarrow P$ has an arbitrary cycle or $m(k)$ pair $\left(p, T p, \ldots, T^{k} p, \ldots, T^{m} p\right)$ of bifurcation points, or that $T: X \rightarrow X$ has a $m(k)$-forks point $p \in X$.

Theorem 34. (Existence of $m(k)$-forked points). Let $T$ be a mapping of a topological space $X$ into itself, where $X$ satisfies the condition of lower ordered RBS-convergence. If

$$
B(T x) \preccurlyeq B(x) \quad \text { for every } \quad x \in X,
$$


then for $T$ there exists an $m(k)$-forked point $\xi \in X$, i.e., then the following equalities hold in the form

(Mm)

$$
B\left(T^{m} \xi\right)=\cdots=B\left(T^{k} \xi\right)=\cdots=B(T \xi)=B(\xi)=\alpha:=\inf _{x \in X} \lim _{n \rightarrow \infty} B\left(b_{n}(x)\right)
$$

for a fixed integer $k \geqslant 1$, for an arbitrary integer $m \geqslant k$, and for some sequence $\left\{b_{n}(x)\right\}_{n \in \mathbb{N}}$ in $X$ which converges to $\xi \in X$.

The elementary proof of this statement is totally analogous with the proof of Theorems 32 and 33. A brief proof of this statement may be found in Ta s k ović [51].

Interpretation and facts. We notice, first, that Theorems 32, 33 and 34 hold even we are to make weaker the condition of lower ordered RBSconvergence, in the sense that this condition holds only for iteration sequences.

In this sense, let $X$ be an arbitrary nonempty set, $T: X \rightarrow X, P:=$ $(P, \preccurlyeq)$ be a nonempty poset, and $B: X \rightarrow P$. A topological space $X$ satisfies the condition of orbital lower ordered RBS-convergence iff $\left\{T^{n}(x)\right\}_{n \in \mathbb{N}}$ is an arbitrary iteration sequence in $X$ with arbitrary $x \in X$ and if $B\left(T^{n}(x)\right) \rightarrow b=b(x) \in P(n \rightarrow \infty)$ implies that $\left\{T^{n}(x)\right\}_{n \in \mathbb{N}}$ has a convergent subsequence $\left\{T^{n(k)}(x)\right\}_{k \in \mathbb{N}}$ which converges to $\xi \in X$, where

$$
B(\xi) \preccurlyeq \inf _{x \in X} \lim _{k \rightarrow \infty} B\left(T^{n(k)}(x)\right) .
$$

Also, we shall introduce the concept of RBS-completeness in a space $X$ for a function $B: X \rightarrow P$, i.e., a topological space $X$ is called ordered RBS-complete (orbital ordered RBS-complete) iff $\left\{a_{n}(x)\right\}_{n \in \mathbb{N}}$ is an arbitrary sequence (an arbitrary iteration sequence) in $X$ with arbitrary $x \in X$ and if $B\left(a_{n}(x)\right) \rightarrow b=b(x) \in P$ as $n \rightarrow \infty$ implies that $\left\{a_{n}(x)\right\}_{n \in \mathbb{N}}$ has a convergent subsequence in $X$.

On the other hand, a function $B: X \rightarrow P$ is lower ordered RBScontinuous (orbital lower ordered $R B S$-continuous) at $p \in X$ iff $\left\{a_{n}(x)\right\}_{n \in \mathbb{N}}$ is an arbitrary sequence (an arbitrary iteration sequence) in $X$ with arbitrary $x \in X$ and if $a_{n}(x) \rightarrow p(n \rightarrow \infty)$ implies that

$$
B(p) \preccurlyeq \inf _{x \in X} \lim _{n \rightarrow \infty} B\left(a_{n}(x)\right) .
$$

Second, we are now in a position to formulate the following explanations of the preceding theorems as corresponding equivalent forms:

Theorem 35. Let $T$ be a mapping of a topological space $X$ into itself and let $X$ be orbital $R B S$-complete. If $(B u)$ holds and if $B: X \rightarrow P$ is an orbital lower ordered $R B S$-continuous map, then for $T$ there exists an $k$-forked point $\xi \in X$.

This result is contained in Theorem 32 as the case for $k=1$, i.e., for the case of a forked point. In this sense we obtain the following result. 
Theorem 36. Let $T$ be a mapping of a topological space $X$ into itself and let $X$ be orbital $R B S$-complete. If (Bu) holds and if $B: X \rightarrow P$ is an orbital lower ordered RBS-continuous map, then for $T$ there exists an $m(k)$-forked point $\xi \in X$.

We also notice as in the preceding case of Theorem 35 that the proof of this statement is a totally analogous with the proof of Theorem 34 which is equivalent to the Theorem 36 for the case of orbital RBS-completeness.

Third, we notice that the dual forms of Theorems 32, 33 and 34 also hold. In this sense, a topological space $X$ satisfies the condition of upper ordered RBS-convergence (orbital upper ordered RBS-convergence) iff $\left\{a_{n}(x)\right\}_{n \in \mathbb{N}}$ is an arbitrary sequence (an arbitrary iterative sequence) in $X$ with arbitrary $x \in X$ and if $B\left(a_{n}(x)\right) \rightarrow b=b(x) \in P(n \rightarrow \infty)$ implies that $\left\{a_{n}(x)\right\}_{n \in \mathbb{N}}$ has a convergent subsequence $\left\{a_{n(k)}(x)\right\}_{k \in \mathbb{N}}$ which converges to $\xi \in X$, where

$$
B(\xi) \succcurlyeq \sup _{x \in X} \lim _{k \rightarrow \infty} B\left(a_{n(k)}(x)\right) .
$$

Adequate with this, a function $B: X \rightarrow P$ is upper ordered RBScontinuous (orbital upper ordered $R B S$-continuous) at $p \in X$ iff $\left\{a_{n}(x)\right\}_{n \in \mathbb{N}}$ is an arbitrary sequence (an arbitrary iteration sequence) in $X$ with arbitrary $x \in X$ and if $a_{n}(x) \rightarrow p(n \rightarrow \infty)$ implies that

$$
B(p) \succcurlyeq \sup _{x \in X} \lim _{n \rightarrow \infty} B\left(a_{n}(x)\right) .
$$

Theorem 37. (Duality of Theorems 32 and 33). Let $T$ be a mapping of a topological space $X$ into itself and let $X$ be orbital ordered $R B S$-complete. If

$$
B(T x) \succcurlyeq B(x) \text { for every } x \in X,
$$

and if $B: X \rightarrow P$ is an orbital upper ordered RBS-continuous map, then for $T$ there exists an $k$-forked point $\xi \in X$, i.e., then the following equalities hold:

$$
B\left(T^{k} \xi\right)=\cdots=B(T \xi)=B(\xi)=\alpha:=\sup _{x \in X} \lim _{n \rightarrow \infty} B\left(b_{n}(x)\right)
$$

for an arbitrary fixed integer $k \geqslant 1$ and for an iteration sequence $\left\{b_{n}(x)\right\}_{n \in \mathbb{N}}$ in $X$ which converges to $\xi \in X$.

This result also contained a dual version of Theorem 32 as the case for $k=1$, i.e., for the case of a forked point $\xi \in X$. Directly, the following result also holds.

Theorem 38. (Duality of Theorem 34). Let $T$ be a mapping of a topological space $X$ into itself and let $X$ be orbital ordered $R B S$-complete. If (Bd) and if $B: X \rightarrow P$ is an orbitally upper ordered $R B S$-continuous map, then for $T$ there exists an $m(k)$-forked point $\xi \in X$. 
In connection with the preceding, from the former results: a function $f: X \rightarrow P$, where $P:=(P, \preccurlyeq)$ is a lattice and $T: X \rightarrow X$, has a $\mathrm{R}$-forked point if and only if

$$
\min \{f(x), f(T x)\} \preccurlyeq f(\xi)=f(T \xi) \preccurlyeq \max \{f(x), f(T x)\}
$$

for some $\xi \in X$ and for every $x \in X$. In this sense we also have the following result as a minimax statement for forked points.

Theorem 39. Let $P:=(P, \preccurlyeq)$ be a conditional complete poset, $X$ and $Y$ be nonempty sets, and $T: X \rightarrow Y$. If $f: X \rightarrow P$ has a minimum and $y \mapsto f(T y)$ has a maximum, then the following equality holds in the form

$$
\min _{x \in X} \sup _{y \in Y} \max \{f(x), f(T y)\}=\max _{y \in Y} \inf _{x \in X} \min \{f(x), f(T y)\}
$$

if and only if for any two finite sets $\left\{x_{1}, \ldots, x_{n}\right\} \subset X$ and $\left\{y_{1}, \ldots, y_{m}\right\} \subset Y$ the following inequality holds in the form

$$
\max \left\{f\left(x_{0}\right), f\left(T y_{k}\right)\right\} \preccurlyeq \min \left\{f\left(x_{i}\right), f\left(T y_{0}\right)\right\}
$$

for some elements $x_{0} \in X$ and $y_{0} \in Y$, for all $i=1, \ldots, n$ and for all $k=1, \ldots, m$.

Proof. Necessity. Let the inequality (46) hold. Then there exist elements $x_{0} \in X$ and $y_{0} \in Y$ such that the following equality holds in the form

$$
\sup _{y \in Y} \max \left\{f\left(x_{0}\right), f(T y)\right\}=\inf _{x \in X} \min \left\{f(x), f\left(T y_{0}\right)\right\} ;
$$

and thus $\max \left\{f\left(x_{0}\right), f\left(T y_{k}\right)\right\} \preccurlyeq \min \left\{f\left(x_{i}\right), f\left(T y_{0}\right)\right\}$ for $i=1,2, \ldots, n$ and $k=1,2, \ldots, m$. This means that the conditions (47) hold.

Sufficiency. According to this condition, from the condition (47), since $P$ is a conditional complete poset, we have the following inequality in the form

$$
\sup _{1 \leq k \leqslant m} \max \left\{f\left(x_{0}\right), f\left(T y_{k}\right)\right\} \preccurlyeq \inf _{1 \leq i \leqslant n} \min \left\{f\left(x_{i}\right), f\left(T y_{0}\right)\right\},
$$

and thus, since $P$ is a conditional complete poset, it follows that there is a supremum of left side and an infimum of right side of this inequality, i.e., we obtain that

$\sup _{m \leqslant \text { Card } Y} \sup _{1 \leq k \leqslant m} \max \left\{f\left(x_{0}\right), f\left(T y_{k}\right)\right\} \preccurlyeq \inf _{n \leqslant \text { Card } X} \inf _{1 \leq i \leq n} \min \left\{f\left(x_{i}\right), f\left(T y_{0}\right)\right\}$.

This is an inequality of two inequalities in (46). Since the second inequality trivially holds, it follows that the equality (46) holds. The proof is complete.

Annotation. We notice, first, that the equality (46) has an equivalent booking via power of sets $X$ and $Y$ in the following form:

$\min _{x \in X} \sup _{m \leqslant \operatorname{Card} Y} \sup _{1 \leq k \leqslant m} \max \{f(x), f(T y)\}=\max _{y \in Y} \inf _{n \leqslant \operatorname{Card} X} \inf _{1 \leq i \leq n} \min \{f(x), f(T y)\}$. 
Also, on the other hand, for $P:=\mathbb{R}$, i.e., if $f: X \rightarrow P$ is a real function, then Theorem 39 has the following booking in the form:

Theorem 40. Let $X$ and $Y$ be two compact Hausdorff, spaces and $T: Y \rightarrow$ $Y$. Suppose that $f: X \rightarrow \mathbb{R}$ is lower semicontinuous on $X$ and $y \mapsto f(T y)$ is upper semicontinuous on $Y$. Then

$$
\min _{x \in X} \max _{y \in Y} \max \{f(x), f(T y)\}=\max _{y \in Y} \min _{x \in X} \min \{f(x), f(T y)\}
$$

if and only if for any two finite sets $\left\{x_{1}, \ldots, x_{n}\right\} \subset X$ and $\left\{y_{1}, \ldots, y_{m}\right\} \subset Y$ there exist $x_{0} \in X$ and $y_{0} \in Y$ such that (47).

A brief proof of this statement, based on a structure of the set of real numbers as a conditional complete poset, may be found in Taskovi ć [51].

Forked points and RBS-convergence. If for the partial ordered set $P:=(P, \preccurlyeq)$ to set $P=\mathbb{R}$, where relation order $\preccurlyeq$ is usual number order $\leqslant$, then on the topological space $X$ and for $B: X \rightarrow \mathbb{R}$ we have the following facts.

A topological space $X$ satisfies the condition of lower RBS-convergence (orbital lower $R B S$-convergence) iff $\left\{a_{n}(x)\right\}_{n \in \mathbb{N}}$ is an arbitrary sequence (an arbitrary iteration sequence) in $X$ with arbitrary $x \in X$ and if $B\left(a_{n}(x)\right) \rightarrow$ $b=b(x) \in \mathbb{R} \cup\{-\infty\}$ as $n \rightarrow \infty$ implies that $\left\{a_{n}(x)\right\}_{n \in \mathbb{N}}$ has a convergent subsequence $\left\{a_{n(k)}(x)\right\}_{k \in \mathbb{N}}$ which converges to $\xi \in X$, where

$$
B(\xi) \leqslant \inf _{x \in X} \liminf _{k \rightarrow \infty} B\left(a_{n(k)}(x)\right) .
$$

In this sense, a topological space $X$ is called lower RBS-complete (orbital lower $R B S$-complete) iff $\left\{a_{n}(x)\right\}_{n \in \mathbb{N}}$ is an arbitrary sequence (an arbitrary iteration sequence) in $X$ with arbitrary $x \in X$ and if $B\left(a_{n}(x)\right) \rightarrow$ $b=b(x) \in \mathbb{R} \cup\{-\infty\}$ as $n \rightarrow \infty$ implies that $\left\{a_{n}(x)\right\}_{n \in \mathbb{N}}$ has a convergent subsequence in $X$.

A function $B: X \rightarrow \mathbb{R}$ is lower RBS-continuous (orbital lower $R B S$ continuous) at $p \in X$ iff $\left\{a_{n}(x)\right\}_{n \in \mathbb{N}}$ is an arbitrary sequence (an arbitrary iteration sequence) in $X$ with arbitrary $x \in X$ and if $a_{n}(x) \rightarrow p(n \rightarrow \infty)$ implies that

$$
B(p) \leqslant \inf _{x \in X} \liminf _{n \rightarrow \infty} B\left(a_{n}(x)\right) .
$$

Theorem 41. (Forked points, Tasković [44]). Let $T$ be a mapping of a topological space $X$ into itself and let $X$ be orbital lower $R B S$-complete. If

$$
B(T x) \leq B(x) \text { for every } x \in X,
$$

and if $B: X \rightarrow \mathbb{R}$ is an orbitally lower RBS-continuous map, then for $T$ there exists an $k$-forked point $\xi \in X$, i.e., the following equalities hold:

$$
B\left(T^{k} \xi\right)=\cdots=B(T \xi)=B(\xi)=\alpha:=\inf _{x \in X} \lim _{n \rightarrow \infty} B\left(b_{n}(x)\right)
$$


for any arbitrary fixed integer $k \geqslant 1$ and for an iteration sequence $\left\{b_{n}(x)\right\}_{n \in \mathbb{N}}$ in $X$ which converges to $\xi \in X$.

This result is also contained in the case $k=1$, i.e., for the case when $T$ has a forked point $\xi \in X$. A brief proof of this statement may be found in Taskovi ć [44].

Theorem 42. ( $m(k)$-forked points). Let $T$ be a mapping of a topological space $X$ into itself and let $X$ be an orbital lower $R B S$-complete. If (B) and if $B: X \rightarrow \mathbb{R}$ is an orbital lower $R B S$-continuous map, then for $T$ there exists an $m(k)$-forked point $\xi \in X$.

A topological space $X$ satisfies the condition of upper RBS-convergence (orbital upper $R B S$-convergence) iff $\left\{a_{n}(x)\right\}_{n \in \mathbb{N}}$ is an arbitrary sequence (an arbitrary iteration sequence) in $X$ with arbitrary $x \in X$ and if $B\left(a_{n}(x)\right) \rightarrow$ $b=b(x) \in \mathbb{R} \cup\{+\infty\}$ as $n \rightarrow \infty$ implies that $\left\{a_{n}(x)\right\}_{n \in \mathbb{N}}$ has a convergent subsequence $\left\{a_{n(k)}(x)\right\}_{k \in \mathbb{N}}$ which converges to $\xi \in X$, where

$$
B(\xi) \geqslant \sup _{x \in X} \limsup _{k \rightarrow \infty} B\left(a_{n(k)}(x)\right) .
$$

In this sense, a topological space $X$ is called upper RBS-complete (orbital upper $R B S$-complete) iff $\left\{a_{n}(x)\right\}_{n \in \mathbb{N}}$ is an arbitrary sequence (an arbitrary iteration sequence) in $X$ with arbitrary $x \in X$ and if $B\left(a_{n}(x)\right) \rightarrow$ $b=b(x) \in \mathbb{R} \cup\{+\infty\}$ as $n \rightarrow \infty$ implies that $\left\{a_{n}(x)\right\}_{n \in \mathbb{N}}$ has a convergent subsequence in $X$.

A function $B: X \rightarrow \mathbb{R}$ is upper RBS-continuous (orbital upper $R B S$ continuous) at $p \in X$ iff $\left\{a_{n}(x)\right\}_{n \in \mathbb{N}}$ is an arbitrary sequence (an arbitrary iteration sequence) in $X$ with arbitrary $x \in X$ and if $a_{n}(x) \rightarrow p(n \rightarrow \infty)$ implies that

$$
B(p) \geqslant \sup _{x \in X} \limsup _{n \rightarrow \infty} B\left(a_{n}(x)\right) .
$$

Theorem 43. (Duality of Theorem 41). Let $T$ be a mapping of a topological space $X$ into itself and let $X$ be orbitally upper $R B S$-complete. If

$$
B(T x) \geqslant B(x) \text { for every } x \in X,
$$

and if $B: X \rightarrow \mathbb{R}$ is an orbital upper $R B S$-continuous map, then for $T$ there exists an $k$-forked point $\xi \in X$, i.e., the following equalities hold:

$$
B\left(T^{k} \xi\right)=\cdots=B(T \xi)=B(\xi)=\alpha:=\sup _{x \in X} \lim _{n \rightarrow \infty} B\left(b_{n}(x)\right)
$$

for any arbitrary fixed integer $k \geqslant 1$ and for an iteration sequence $\left\{b_{n}(x)\right\}_{n \in \mathbb{N}}$ in $X$ which converges to $\xi \in X$.

This result also contained the case $k=1$, i.e., for the case when $T$ has a forked point $\xi \in X$. The following result also holds in the preceding sense. 
Theorem 44. (Duality of Theorem 42). Let $T$ be a mapping of a topological space $X$ into itself and let $X$ be orbital upper $R B S$-complete. If (D) and if $B: X \rightarrow \mathbb{R}$ is an orbital upper RBS-continuous map, then for $T$ there exists an $m(k)$-forked point $\xi \in X$.

\section{Consequences of the diametral $\varphi$-COntraction}

Diametral $\varphi$-contraction on metric spaces. In 1980 I proved the following result of a fixed point on metric space which has for the best long of all known sufficient conditions (linear and nonlinear) for the existence of a unique fixed point, cf. T a s k o v i ć [35], [52], and [38]. This result generalizes a great number of known results.

Theorem 45. (Tasković, [35]). Let $T$ be a mapping of a metric space $(X, \rho)$ into itself and let $X$ be T-orbital complete. Suppose that there exists a function $\varphi: \mathbb{R}_{+}^{0} \rightarrow \mathbb{R}_{+}^{0}:=[0,+\infty)$ satisfying

$$
\left(\forall t \in \mathbb{R}_{+}:=(0,+\infty)\right)\left(\varphi(t)<t \quad \text { and } \quad \limsup _{z \rightarrow t+0} \varphi(z)<t\right)
$$

such that the following inequality holds in the form

$$
\rho[T x, T y] \leqslant \varphi\left(\operatorname{diam}\left\{x, y, T x, T y, T^{2} x, T^{2} y, \ldots\right\}\right)
$$

for all $x, y \in X$. If $\operatorname{diam} \mathcal{O}(x) \in \mathbb{R}_{+}^{0}$ for every $x \in X$, then $T$ has a unique fixed point $\xi \in X$ and $\left\{T^{n}(a)\right\}_{n \in \mathbb{N}}$ converges to $\xi$ for every $a \in X$.

In connection with this result, we notice that this statement is well-known as: "the finest theorem of nonlinear functional analysis" for metric spaces.

In thes context of this statement, the following conditions (linear and nonlinear) are special cases of the diametral $\varphi$-contraction on complete metric space $(X, \rho)$, i.e., of the condition $(\mathrm{J})$ in Theorem 45 :

(1) (B a n a c h, [1922]). There exists a number $\lambda \in[0,1)$ such that for all point $x, y \in X$ the following inequality holds in the form

$$
\rho[T(x), T(y)] \leqslant \lambda \rho[x, y] .
$$

(2) (K a n n a n, [1968]). There exists a number $\alpha \in[0,1 / 2)$ such that for all points $x, y \in X$ the following inequality holds in the form

$$
\rho[T(x), T(y)] \leqslant \alpha(\rho[x, T(x)]+\rho[y, T(y)]) .
$$

(3) (Reich [1971], R u s [1971]). There exist nonnegative numbers $a, b, c$ satisfying the following inequality $a+b+c<1$ such that for all $x, y \in X$ the following inequality holds in the form

$$
\rho[T x, T y] \leq a \rho[x, T x]+b \rho[y, T y]+c \rho[x, y] .
$$

(4) (R a k o t c h, [1962]). There exists a monotone decreasing function $\alpha: \mathbb{R}_{+} \rightarrow$ $[0,1)$ such that for all $x, y \in X(x \neq y)$ the following inequality holds in the form

$$
\rho[T(x), T(y)] \leqslant \alpha(\rho[x, y]) \rho[x, y] .
$$


(5) (K r a s n o s els ki j et al., [1973]). There exists a nonnegative function $\beta$ that satisfies the following inequality in the form $\sup \{\beta(x, y): a \leqslant \rho[x, y] \leq b\}<$ 1 for each finite closed interval $[a, b] \subset \mathbb{R}_{+}$and

$$
\rho[T(x), T(y)] \leqslant \beta(x, y) \rho[x, y] \text { for all } x, y \in X .
$$

(6) ( $\mathrm{R}$ h o a d e s [1977], C h a t t e r j e a [1972]). There exists a number $\alpha \in$ $[0,1)$ such that for all points $x, y \in X$ the following inequality holds in the form

$$
\rho[T(x), T(y)] \leqslant \alpha \max \{\rho[x, T(y)], \rho[y, T(x)]\} .
$$

(7) (H a r d y-R o g e r s [1973], I s é k i [1975], K u r e p a [1972]). There exist nonnegative constants $a_{i}(i=1, \ldots, 5)$ satisfying $a_{1}+a_{2}+a_{3}+a_{4}+a_{5}<1$ such that for all $x, y \in X$ the following inequality holds in the form

$$
\rho[T x, T y] \leqslant a_{1} \rho[x, y]+a_{2} \rho[x, T x]+a_{3} \rho[y, T y]+a_{4} \rho[x, T y]+a_{5} \rho[y, T x] .
$$

Annotation. In connection with this inequality, we notice that $\mathrm{T}$ a s k o v i ć [1971] considered a special case of this condition. Also see: K u r e p a [1976], M a t kow ski [1973], and R us [1979]. Interesting, in $1976 \mathrm{~K} \mathrm{u} \mathrm{r} \mathrm{e} \mathrm{p} \mathrm{a} \mathrm{proved}$ a geometric interpretation of the condition (7).

(8) (K u r e p a [1972], M a s s a [1974]). There exists a constant $q \in[0,1)$ such that for all points $x, y \in X$ the following inequality holds in the form

$$
\rho[T x, T y] \leq q \max \{\rho[x, y], \rho[x, T x], \rho[y, T y], \rho[x, T y], \rho[y, T x]\} .
$$

(9) (F i s h e r, [1975]). There exists a constant $\alpha \in[0,1)$ such that for all points $x, y \in X$ the following inequality holds in the form

$$
\rho\left[T^{2}(x), T(y)\right] \leqslant \alpha \max \left\{\rho\left[T(x), T^{2}(x)\right], \rho[y, T(y)]\right\} .
$$

(10) (F i s h e r, [1975]). There exists a constant $\alpha \in[0,1)$ such that for all points $x, y \in X$ the following inequality holds in the form

$$
\rho\left[T^{2}(x), T(y)\right] \leqslant \alpha \max \left\{\rho[T(x), T(y)], \rho\left[y, T^{2}(x)\right]\right\} .
$$

(11) (D u g u n d j i [1976], B i a n ch in i [1972]). There exists a number $\alpha \in$ $[0,1)$ such that for all $x, y \in X$ the following inequality holds in the form

$$
\rho[T(x), T(y)] \leqslant \alpha \max \{\rho[x, T(x)], \rho[y, T(y)]\} .
$$

(12) (J a g g i, [1977]). There exist nonnegative numbers $\alpha, \beta \in[0,1)$ satisfying $\alpha+\beta<1$ such that for all $x, y \in X(x \neq y)$ the following inequality holds in the form

$$
\rho[T x, T y] \leqslant \alpha \rho[x, T x] \rho[y, T y](\rho[x, y])^{-1}+\beta \rho[x, y] .
$$

(13) (P o p a, [1983]). There exist numbers $\alpha, \beta \in[0,1)$ such that $\alpha+\beta<1$ and function $f$ of $X \times X$ into $\mathbb{R}_{+}^{0}$ such that for all $x, y \in X(x \neq y)$ satisfying $f(x, y) \neq 0, f(x, x) f(y, y) \leq f^{2}(x, y)$, and

$$
f(T x, T y) \leqslant \alpha f(x, T x) f(y, T y)(f(x, y))^{-1}+\beta f(x, y) .
$$


(14) (Tasković, [1976]). There exist real numbers $\alpha_{i}(i=1, \ldots, 4)$ and $\beta$ satisfying that $\alpha_{1}+\alpha_{2}+\alpha_{3}>\beta$ and $\beta-\alpha_{2} \geqslant 0$ or $\beta-\alpha_{3} \geqslant 0$ such that for all $x, y \in X$ the following inequality holds in the form

(H)

$$
\alpha_{1} \rho[T x, T y]+\alpha_{2} \rho[x, T x]+\alpha_{3} \rho[y, T y]+\alpha_{4} \min \{\rho[x, T y], \rho[y, T x]\} \leqslant \beta \rho[x, y] .
$$

(15) (I va nov, [1974]). There exist $a, b, c, d \in \mathbb{R}$ satisfying $a+b+2 c<$ $\min \{0,-2 d\}$ and $b+c+d<0$ such that for all $x, y \in X$ the following inequality holds in the form

$$
a \rho[x, y]+b \rho[T x, T y]+c(\rho[x, T x]+\rho[y, T y])+d(\rho[x, T y]+\rho[y, T x]) \geqslant 0 .
$$

Annotation. We notice that the condition (15) is a special case of (14). Indeed, if in $(\mathrm{H})$ we set that is $a=\beta, b=-\alpha_{1}, c=-\alpha_{2}=-\alpha_{3}$, and $d=-\alpha_{4}$, then we obtain that the condition (14) is satisfied, Reverse is not held by an example in Tas k ovi ć [1976].

(16) (H e g e d ü s-S c i l a g y i, [1980]). There exists a function $f: \mathbb{R}_{+}^{0} \rightarrow[0,1)$ such that for every $\varepsilon>0$ there exists $\delta>0$ satisfying $\sup \{f(t): \varepsilon \leqslant t<\varepsilon+\delta\}<1$ and for all $x, y \in X$ the following inequality holds in the form

$$
\rho[T(x), T(y)] \leq f(\rho[x, y]) \rho[x, y] .
$$

(17) (E d elstei n, [1962]). The mapping $T: X \rightarrow X$ is said to be strict contractive if for all $x, y \in X(x \neq y)$ the following inequality holds in the form

$$
\rho[T(x), T(y)]<\rho[x, y] .
$$

(18) (Fr e u d e n t h a l-H u r e w i c z, [1936]). The mapping $T: X \rightarrow X$ is said to be nonexpansive if for all $x, y \in X$ the following inequality holds in the form as

$$
\rho[T(x), T(y)] \leqslant \rho[x, y] .
$$

(19) (B a i le y, [1966]). The mapping $T: X \rightarrow X$ is said to be weakly contractive if for all $x, y \in X(x \neq y)$ there is a positive integer $n=n(x, y)$ such that

$$
\rho\left[T^{n}(x), T^{n}(y)\right]<\rho[x, y] .
$$

(20) (K i r k, [1969]). The mapping $T: X \rightarrow X$ is said to be diminishing orbital diameters if for every $x \in X$ the following inequality holds in the form as

$$
\operatorname{diam} \mathcal{O}(x)>\lim _{n \rightarrow \infty} \operatorname{diam} \mathcal{O}\left(T^{n} x\right), \quad \text { whenever } \quad \operatorname{diam} \mathcal{O}(x) \in \mathbb{R} .
$$

(21) (B e ll u c e-K i r k, [1969]). The mapping $T: X \rightarrow X$ is said to have shrinking orbits if for each $x \in X$ with diam $\mathcal{O}(x) \in \mathbb{R}$ there exists an integer $n \in \mathbb{N}$ such that the following inequality holds in the form as

$$
\operatorname{diam} \mathcal{O}\left(T^{n}(x)\right)<\operatorname{diam} \mathcal{O}(x) .
$$

(22) (Kr a s nos e ls k i j-Z a breiko, [1975]). There exists a function $p$ : $\mathbb{R}_{+} \times \mathbb{R}_{+} \rightarrow[0,1)$ satisfying for all $x, y \in X$ the following inequality in the form

$$
\rho[T(x), T(y)] \leq p(a, b) \rho[x, y]
$$


where $0<a \leqslant \rho[x, y] \leq b$ and $0 \leq p(a, b)<1$ for arbitraries $a, b \in \mathbb{R}_{+}$. (This condition is very connection with the condition (5)).

An application. (Contractive maps on compact sets). Every contractive compact subset $M$ of a metric space $(X, \rho)$ has exactly one fixed point. The sequence of iterates $\left\{x_{n}\right\}_{n \in \mathbb{N} \cup\{0\}}$ defined by $x_{n+1}=T\left(x_{n}\right)$ converges to the fixed point from an arbitrary initial point $x_{0} \in M$.

Proof. (We use that: if (22) holds on complete metric space $(X, \rho)$, then $T$ has exactly one fixed point). By hypothesis, (17) holds, i.e., $\rho[T x, T y]<\rho[x, y]$ for all $x, y \in M(x \neq y)$. Set $f(x, y)=\rho[T x, T y] / \rho[x, y]$ and

$$
S=\{(x, y) \in M \times M: a \leqslant \rho[x, y] \leq b\}, \quad 0<a<b
$$

then the function $f$ is a continuous on the compact set $S$. Therefore, $f$ attains its maximum, denoted by $k(a, b)$. Consequently, $T: M \rightarrow M$ satisfies (22), and thus $T$ has a unique fixed point $\xi \in M$. Since $M$ is compact, $\left\{x_{n}\right\}_{n \in \mathbb{N} \cup\{0\}}$ has a convergent subsequence. From $x_{n+1}=T\left(x_{n}\right)$ it follows that each convergent subsequence of $\left\{x_{n}\right\}_{n \in \mathbb{N} \cup\{0\}}$ has the fixed point $\xi$ as a limit point. Thus, $\left\{x_{n}\right\}_{n \in \mathbb{N} \cup\{0\}}$ converges to $\xi \in M$.

(23) (C h a k r a b a r t y, [1978]). There exists a continuous from the right nondecreasing function $\varphi: \mathbb{R}_{+}^{0} \rightarrow \mathbb{R}_{+}^{0}$ satisfying $\varphi(t)<t$ for every $t>0$, and a continuous function $\psi: X \times X \rightarrow \mathbb{R}_{+}^{0}$ with properties: $\psi(x, x)=0, \rho[x, y] \leqslant \psi(x, y)$ for all $x, y \in X$, and

$$
\psi(T x, T y) \leq \varphi(\psi(x, y)) \text { for all } x, y \in X
$$

where for every $x \in X$ there is an $A \in \mathbb{R}_{+}$such that the following holds in the form $\psi\left(x, T^{n} x\right)<A(x)$.

(24) (B o s e-M u k h e r j e e, [1982]). Let $F: X \times X \rightarrow \mathbb{R}_{+}^{0}$ be a continuous function with properties: $F(x, y)=0$ if and only if $x=y$ and $F(x, y)=F(y, x)$. There exist nonnegative constants $a$ and $b$ satisfying $a+b<1$ such that for all $x, y \in X(x \neq y)$ the following inequality holds in the form

$$
F(T x, T y) \leq a F(x, T x) F(y, T y)(F(x, y))^{-1}+b F(x, y) .
$$

(25) (D h a g e, [1985]). There exist real numbers $a, p, q$ satisfying $0 \leq p+q<1$ such that for all $x, y \in X$ the following inequality holds in the form

$\min \{\rho[T x, T y], \rho[x, T x], \rho[y, T y]\}+a \min \{\rho[x, T y], \rho[y, T x]\} \leqslant p \rho[x, y]+q \rho[x, T x]$.

(26) (B r ow d e r [1968], B oy d-Wong [1969]). There exists a real upper semicontinuous from the right function $\psi: \mathbb{R}_{+}^{0} \rightarrow \mathbb{R}_{+}^{0}$ satisfying $\psi(t)<t$ for every $t>0$ such that

$$
\rho[T(x), T(y)] \leq \psi(\rho[x, y]) \quad \text { for all } \quad x, y \in X .
$$

Annotation. We notice that first in 1968 B r o w d e r established a fixed point theorem for a self-map $T$ on a complete bounded metric space $(X, \rho)$ satisfying continuous from the right function such that $\psi(t)<t$ for every $t \in \mathbb{R}_{+}$.

Also, B r o w d e r [1968] showed that in the case when $X$ is unbounded, a fixed point theorem if $\psi: \mathbb{R}_{+}^{0} \rightarrow \mathbb{R}_{+}^{0}$ fulfills the following additional condition in the form: $t-\psi(t) \rightarrow \infty(t \rightarrow \infty)$. 
This reference (B r ow d e r [1968]) contains also applications to differential equations in Banach spaces. Further variants of the Banach fixed point theorem are also contained in: Collatz [1964], Istrates cu [1973], M i c z ko P a l c z e w s i [1985], and B r ow d e r [1979].

We notice that in Browder's theorem the iterative sequence $x_{n+1}=T\left(x_{n}\right)$ for $n \in \mathbb{N} \cup\{0\}$ converges to $\xi \in X$ from an arbitrary initial point $x_{0} \in M \subset X$, where $M$ is a nonempty bounded subset, and $\rho\left[x_{n}, \xi\right] \leq \psi^{n-1}(\operatorname{diam} M)$ for $n \in \mathbb{N}$. In this case $\psi^{k}$ is the $k$ th iteration, i.e., $\psi^{2}(r)=\psi(\psi(r))$, etc.

(27) (Wo n g, [1968]). There exists a real continuous from the right function $\psi: \mathbb{R}_{+}^{0} \rightarrow \mathbb{R}_{+}^{0}$ satisfying: $\psi(t)<t$ for every $t>0, \psi(0)=0$, and $\liminf _{t \rightarrow 0}(t-$ $\psi(t))=\alpha>0$, such that for every $u \in X$ there is a positive number $n=n(u)$ with property

$$
\rho\left[T^{n}(x), T^{n}(y)\right] \leq \psi(\rho[x, y]) \quad \text { for all } \quad x, y \in \mathcal{O}(n) .
$$

We notice that, in Browder's case, the function $\psi: \mathbb{R}_{+}^{0} \rightarrow \mathbb{R}_{+}^{0}$ satisfies all conditions as in (26).

Annotation. We notice that B r ow der [1968] proved a statement for a special case of Wong's class of functions. It is the case when there exists a constant $n=n(u)$ in the condition (27).

(28) (M e i r-K e e l e r, [1969]). For any $\varepsilon>0$ there exists $\delta(\varepsilon)>0$ such that for all $x, y \in X$ the following fact holds in the form

$$
\rho[T x, T y]<\varepsilon \quad \text { whenever } \varepsilon \leqslant \rho[x, y]<\varepsilon+\delta(\varepsilon) .
$$

(29) (M e i r-K e e l e r [1969], P a r k-K i m [1984]). For any $\varepsilon>0$ there exists a positive number $\varepsilon_{0}<\varepsilon$ and $\delta>0$ such that for all $x, y \in X$ the following fact holds in the form

$$
\rho[T x, T y]<\varepsilon_{0} \quad \text { whenever } \quad \varepsilon \leqslant \rho[x, y]<\varepsilon+\delta .
$$

(30) (C. S.Wo n g, [1976]). There exists a lower semicontinuous function $f$ : $\mathbb{R}_{+}^{0} \rightarrow \mathbb{R}_{+}^{0}$ satisfying $f(s)>s$ for every $s>0$ such that the following inequality holds in the form

$$
f(\rho[T x, T y]) \leq \rho[x, y] \text { for all } x, y \in X .
$$

Annotations. We notice that W o n g [1976] proved that the following conditions are equivalent: (26), (28), and (30). For this see and T a s k ovi ć [1993]. In connection with this is also the following result.

Lemma 1. (Wong, [1976]). Let $h$ be a self map on $\mathbb{R}_{+}^{0}$ such that $h(0)=0, h(t)<t$ for every $t>0$, and $h$ is upper semicontinuous. Then there exists a self map $\psi$ on $\mathbb{R}_{+}^{0}$ such that $\psi(0)=0, \psi(t)<t$ for every $t>0$, and $\psi$ is increasing continuous such that $h(t)<\psi(t)$ for every $t>0$.

(31) (D a n e š [1976], Ta s k ovi ć [1978]). There exists a nondecreasing function $\psi: \mathbb{R}_{+}^{0} \rightarrow \mathbb{R}_{+}^{0}$ satisfying: $\psi^{n}(t) \rightarrow 0(n \rightarrow \infty, t>0)$, and $(t-\psi(t)) \rightarrow+\infty$ $(t \rightarrow \infty)$, such that the following inequality holds in the form

$$
\rho[T x, T y] \leqslant \psi(\operatorname{diam}\{x, y, T x, T y\}) \text { for all } x, y \in X
$$

Annotation. We notice that the condition (DT) as original appeared in Ta s k ovi ć [1978]. On the other hand, the conditions in D a n eš [1976] are different and are in connection with the function of the form $\psi:\left(\mathbb{R}_{+}^{0}\right)^{5} \rightarrow \mathbb{R}_{+}^{0}$ with 
the preceding properties. Also see and: H e g e d ü s-S z i l a g u i [1980], R u s [1979], M a t k ow ski [1977], and Tasković [1975].

(32) (M a t k ow s k i [1977], T a s k ović [1990]). There exists a nondecreasing function $\psi: \mathbb{R}_{+}^{0} \rightarrow \mathbb{R}_{+}^{0}$ with the properties as in (31) such that for every $x \in X$ there exists a positive integer $n=n(x)$ such that the following inequality holds in the form

$$
\rho\left[T^{n} x, T^{n} y\right] \leqslant \psi\left(\operatorname{diam}\left\{x, y, T^{n} x, T^{n} y\right\}\right) \quad \text { for every } \quad y \in X .
$$

Annotation. We notice that the condition (MT) as original appeared in Tas k ovi ć [1990]. On the other hand, the conditions in M a t k ow s k i [1977] are different and are in connection with the function of the form $\psi:\left(\mathbb{R}_{+}^{0}\right)^{5} \rightarrow \mathbb{R}_{+}^{0}$ with the preceding properties.

(33) (C h e h-Y e h, [1978]). There exists a number $\alpha \in[0,1)$ such that for some $n \in \mathbb{N}$ the following inequality holds in the form

$$
\rho\left[T^{n} x, T^{n} y\right] \leqslant \alpha \operatorname{diam}\left\{x, y, T^{n} x, T^{n} y\right\} \quad \text { for all } \quad x, y \in X .
$$

(34) (I v a n o v, [1976]). There exists a real nondecreasing continuous from the right function $\psi: \mathbb{R}_{+}^{0} \rightarrow \mathbb{R}_{+}^{0}$ satisfying $\psi(t)<t$ for every $t>0$, and $(t-\psi(t)) \rightarrow$ $+\infty(t \rightarrow \infty)$, such that for all $x, y \in X$ the following inequality holds in the form

$$
\rho[T x, T y] \leqslant \max \{\psi(\rho[x, y]), \psi(\rho[x, T x]), \psi(\rho[y, T y]), \psi(\rho[x, T y]), \psi(\rho[y, T x])\} .
$$

Annotation. Since the function $\psi: \mathbb{R}_{+}^{0} \rightarrow \mathbb{R}_{+}^{0}$ is nondecreasing, directly, by (I) we obtain that the condition (34) implies the condition $(\mathrm{J})$ in Theorem 82 .

(35) (T a s k o vi ć, [1980]). There exists a nondecreasing function $\psi: \mathbb{R}_{+}^{0} \rightarrow \mathbb{R}_{+}^{0}$ satisfying $\lim \sup _{z \rightarrow t+0} \psi(z)<t$ for every $t>0$ such that the following inequality holds in the form

$$
\rho[T x, T y] \leqslant \psi\left(\operatorname{diam}\left\{x, y, T x, T y, \ldots, T^{k} x, T^{m} y\right\}\right)
$$

for arbitrary fixed integers $k, m \geqslant 0$ and for all points $x, y \in X$. (This is a nonlinear condition for diameter of finite number of points).

Annotation. This nonlinear case has the following geometrical interpretation on the Figure 8. For a given 2-point set $\{x, y\} \subset X$ and $m=k=0$ we consider the corresponding complete graph $\{x, y, T x, T y\} \subset X$. We also, give the cases $m=k=1$, and $m=k=2$.

(36) (D a r b o [1955], F u r i-V i g n o li [1969]). The continuous mapping $T$ is called densifying, if for every bounded subset $A$ of $X$,such that $\alpha(A)>0$, we have $\alpha(T(A))<\alpha(A)$, where $\alpha$ is measure of noncompactness. Let $F$ be a real lower semicontinuous function defined on $X \times X$. The densifying mapping $T$ is said to be weak $F$-contractive if the condition $F(T x, T y)<F(x, y)$ holds for all $x, y \in X$ $(x \neq y)$.

(37) (R a y-C h a t t e r j e e [1977], S in g h [1969], K h a n [1980]). Let $F$ : $X \times X \rightarrow \mathbb{R}_{+}^{0}$ be continuous and $T: X \rightarrow X$ be a densifying mapping such that $F(T x, T y)<a F(x, y)+b F(x, T x)+c F(y, T y)$ for each pair of distant points $x, y \in X$ and for nonnegative real numbers $a, b, c$ with $a+b+c<1$, then $T$ is called generalized densifying. 


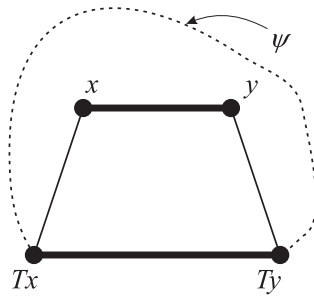

$m=k=0$

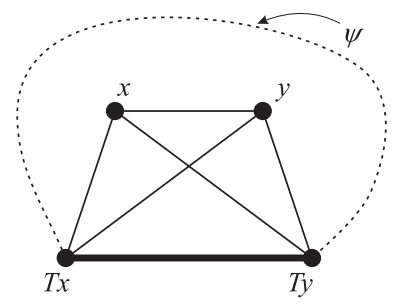

$m=k=1$

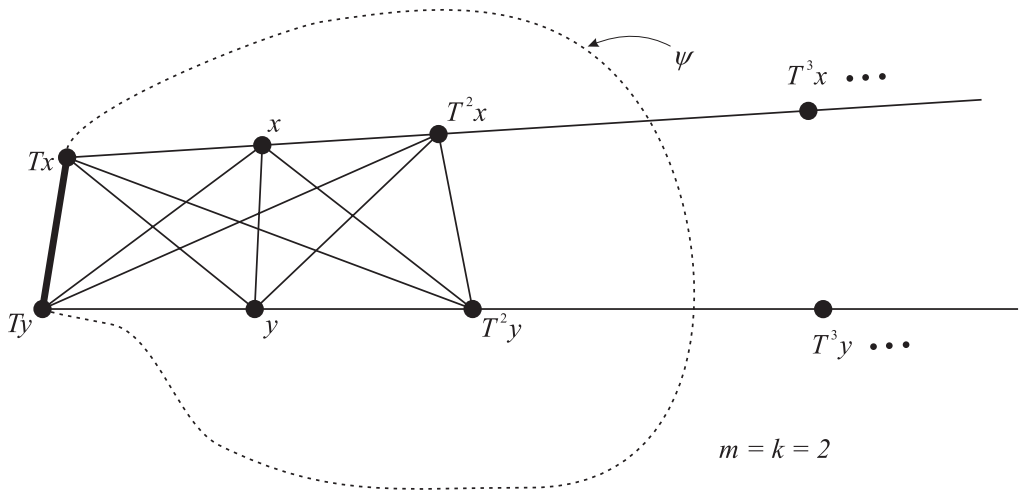

FIGURE 8

(38) (W a l t e r, [1981]). There exists a nondecreasing continuous function $\psi$ : $\mathbb{R}_{+}^{0} \rightarrow \mathbb{R}_{+}^{0}$ satisfying $\psi(t)<t$ for every $t>0$ such that for all $x, y \in X$ the following inequality holds in the form

$$
\rho[T x, T y] \leqslant \psi\left(\operatorname{diam}\left\{x, y, T x, T y, T^{2} x, T^{2} y, \ldots\right\}\right) .
$$

Annotation. This special case of the Tasković's condition in 1980 appeared one year later in Walter as an answer to Browder's result in 1979. But, both conditions (B r ow d e r [1979], and Wa lt e r [1981]) are very special cases of $(\mathrm{J})$ and $(\mathrm{I} \varphi)$ which are give by Tas k ovi ć [1980].

(39) (Linear case of (J): Ta s k o vi ć [1980], and H e g e d ü š [1980]). There exists a number $\alpha \in[0,1)$ such that for all points $x, y \in X$ the following inequality holds in the form

$$
\rho[T x, T y] \leqslant \alpha \max \left\{x, y, T x, T y, T^{2} x, T^{2} y, \ldots\right\} .
$$

(40) (A k k o u ch i, [2002]). There exists a nondecreasing continuous function $\psi: \mathbb{R}_{+}^{0} \rightarrow \mathbb{R}_{+}^{0}$ satisfying that $x \mapsto x-\psi(x): \mathbb{R}_{+}^{0} \rightarrow \mathbb{R}_{+}^{0}$ is a strictly increasing function such that for all $x, y \in X$ the following inequality holds in the form

$$
\rho[T x, T y] \leqslant \psi\left(\operatorname{diam}\left\{x, y, T x, T y, T^{2} x, T^{2} y, \ldots\right\}\right) .
$$

Annotation. We notice that this condition appeared for the first time 27 years ago as the condition $(\mathrm{J})$ in Tas k ovi ć [1980]. But, the author is to neglect and 
ignore this historical fact! Also, K i r k-S a l i g a [2000] are to ignore this historical fact.

(41) (T a s k o vi ć, [1978]). There exists a nondecreasing function $\psi: \mathbb{R}_{+}^{0} \rightarrow \mathbb{R}_{+}^{0}$ satisfying $\lim \sup _{z \rightarrow t+0} \psi(z)<t$ for every $t>0$ such that for all $x, y \in X$ the following inequality holds in the form

$$
\rho[T x, T y] \leqslant \psi(\operatorname{diam}\{x, y, T x, T y\}) .
$$

Without any additional assumptions on $X$ or the mapping $T$, the following sequence of implications hold between the various distance diminishing properties (linear and nonlinear) given above:

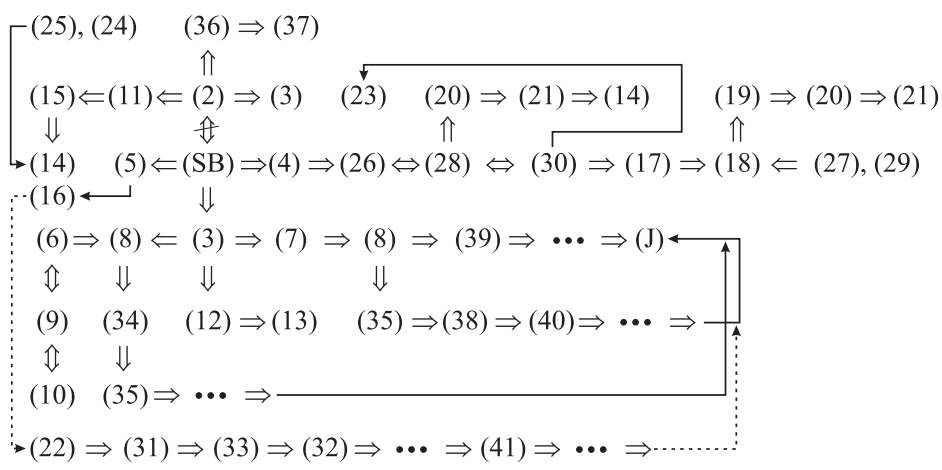

\section{Principles of Transpose}

This part presents some new principles in Functional Analysis. Let $X$ be a nonemty set. Let $\mathcal{C}$ be an arbitrary formula which contains terms $x, y \in X, f_{i}: X \rightarrow X(i=1, \ldots, k \in \mathbb{N})$, where $k \in \mathbb{N}$ is a fixed number, and $\rho: X \times X \rightarrow \mathbb{R}_{+}^{0}$ or $\rho: X \times X \rightarrow[a, b) \in G$ as $a \prec b$ for fixed elements $a, b \in P:=(P, \preccurlyeq)$, where $P$ is an arbitrary partially ordered set and $G$ is a set of all intervals of the form $[a, b)$ for $a, b \in P$ or $a, b \in[0,+\infty]$, satisfying that every decreasing sequence $\left\{u_{n}\right\}_{n \in \mathbb{N}}$ of elements in $[a, b)$ has a unique element $u \in[a, b)$ as limit. Applying Axiom of Choice we obtain the following statement.

Theorem 46. (Principle of Transpose). Let $X$ be a nonempty set, $\mathcal{C}$ an arbitrary formula and let $f_{i}: X \rightarrow X(i=1, \ldots, k \in \mathbb{N})$. Then an assertion of the form: For all $f_{i}(1, \ldots, k)$ and for every $\rho \in \mathbb{R}_{+}^{0}$ the fact

$$
\mathcal{C}\left(x, y \in X, f_{i}(i=1, \ldots, k), \rho\right) \quad \text { implies } f_{i}(i=1, \ldots, k)
$$


is a theorem if and only if the assertion of the form: For all $f_{i}(i=1, \ldots, k)$ and for every $\rho \in[a, b) \in G$ the following fact of the form

$$
\mathcal{C}\left(x, y \in X, f_{i}(i=1, \ldots, k), \rho\right) \text { implies } f_{i}(i=1, \ldots, k)
$$

\section{have a coincidence point}

is a theorem. (A local form of this statement, denoted as: Local Principle of Transpose, is in the case if formulas (A) and (TA) hold only via term $x \in X)$.

A brief proof of this principle based on Axiom of Choice may be found in Tas k ovi ć [51]. The fact that (A) implies (TA), however, may be proved independently and without using the Axiom of Choice!

History. The concept of an abstract metric space, introduced by M. Fré c h e t in 1905, furnishes the common idealization of a large number of mathematical, physical and other scientific constructs in which the notion of a distance appears.

The objects under consideration may be most varied. The may be points, functions, sets, and even the subjective experiences of sensations. A generalization which was first introduced by K. M e n g e r in 1942 and, following him, is called a statistical metric space.

In 1934 Đ. K u r e p a defined pseudodistancional spaces, with the nonnumerical distance, which play an important role in nonlinear numerical analysis (see: L. Collat z [27]). After that several authors investigated the distance functions taking values in partially ordered sets (A. A p p e r t, M. Fréch et, J. C ol m e z, R. D o s s, Ky Fan, and others in the year's 40's and 50's).

Concept of transversal spaces with the nonnumerical transverse were introduced in 1998 by Tasković as a nature extension of Fréchet's, K urepa's, and Menger's spaces in well-know sense. The transversal spaces play an important role in nonlinear functional analysis as and in numerical analysis.

An example of pseudodistancial (as and transversal) spaces is so-called cone of a metric space (or cone metric space). For the cone metric space we formulate principles of transpose.

Let $E:=(E,+)$ be a topological vector space. A subset $P$ of $E$ is called a cone iff $P$ is a closed, nonempty and $P \neq\{0\} ;$ if $a, b \in \mathbb{R}(a, b \geq 0)$ and $x, y \in P$ then $a x+b y \in P$; and $P \cap(-P)=\{0\}$.

For a given cone $P \subset E$, we define a partial ordering $\preccurlyeq$ with respect to $P$ by $x \preccurlyeq y$ if and only if $y-x \in P$. We shall write $x \prec y$ if $x \preccurlyeq y$ and $x \neq y$; also, $x \preccurlyeq y$ means that $y-x \in$ int $P$, where int $P$ denotes the interior of $P$.

The cone $P$ is called normal if there is a number $\sigma>0$ such that for all $x, y \in E$ we have $\|x\| \leq \sigma\|y\|$ whenever $\theta \preccurlyeq x \preccurlyeq y$.

Let $X$ be a nonempty set. In this sense, suppose that the mapping $\rho$ : $X \times X \rightarrow P \subset E$ has all the metric axioms (i.e., $\rho[x, y]=\theta:=0$ if and 
only if $x=y, \rho[x, y]=\rho[y, x]$, and $\rho[x, y] \preccurlyeq \rho[x, z] \oplus \rho[z, y]$ as in the papers: Kurepa [65], Collatz [27], or Sikorski [66]).

In the same manner, $\rho$ is called a cone metric on $X$, and $(X, \rho, \oplus)$ is called cone metric space, where $\oplus=+$ in the topological vector space $E$. Thus $\rho$ satisfies all the axioms of transvsersal spaces with the nonnumerical transverse (as and all axioms of Kurepa's pseudodistantial spaces, see: Kurepa [65]).

Taking one consideration with another, as an immediate fact from the preceding statement, we have directly the following result for cone metric spaces.

Theorem 47. (Cone Principle of Transpose). Let $X$ be a nonempty set and let $\mathcal{C}$ be an arbitrary formula which contains terms $x, y \in X, \leq,+, \preccurlyeq, \oplus$, $f_{i}: X \rightarrow X(i=1, \ldots, k)$ for a fixed number $k \in \mathbb{N}$, and $\rho$. Then, an assertion of the form: For every $f_{i}(i=1, \ldots k)$ and for every $\rho(x, y) \in \mathbb{R}_{+}^{0}$ the following fact in the form

$$
\mathcal{C}\left(x, y \in X, \leq,+, f_{i}(i=1, \ldots, k), \rho\right) \text { implies } f_{i}(i=1, \ldots, k)
$$

have a coincidence point

is a theorem if and only if the assertion of the form: For every $f_{i}(i=$ $1, \ldots, k)$ and for every $\rho(x, y) \in C$, where $C$ is a cone of the set $G$ of all cones, the following fact in the form

$$
\mathfrak{C}\left(x, y \in X, \preccurlyeq, \oplus, f_{i}(i=1, \ldots, k), \rho\right) \text { implies } f_{i}(i=1, \ldots, k)
$$

have a coincidence point

is a theorem. (The local form of this statement we obtain whenever $(E)$ and (R) hold only via term of the form $x \in X$ ).

We notice that in the preceding statement if facts (E) and (R) to substitute with the following facts in the forms as

$$
\mathfrak{C}\left(x, y \in X, \leq,+, f_{i}(i=1, \ldots, k), \rho\right) \text { implies } \mathfrak{M}\left(f_{i}\right),
$$

and

$$
\mathcal{C}\left(x, y \in X, \preccurlyeq, \oplus, f_{i}(i=1, \ldots, k), \rho\right) \quad \text { implies } \mathfrak{M}\left(f_{i}\right),
$$

respectively, where the property $\mathfrak{M}\left(f_{i}\right)$ is a form of: 1$) f_{i}(i=1, \ldots, k)$ have a common fixed point, 2$)$ there exists a countable sequence which converges to a common fixed point of $f_{i}(i=1, \ldots, k)$.

We are now in a position to formulate our next theorems as directly consequences of principle of transpose for cone metric spaces and further. 
Theorem 48. Let $X$ be a nonempty set and let $\mathfrak{C}$ be an arbitrary formula which contains terms $x, y \in X, \leq,+, \preccurlyeq, \oplus, T: X \rightarrow X$ and $\rho$. Then, an assertion of the form: For every $T$ and for every $\rho(x, y) \in \mathbb{R}_{+}^{0}$ the following fact in the form

$$
\mathcal{C}(x, y \in X, \leq,+, T, \rho) \text { implies } T \text { has a fixed point }
$$

is a theorem if and only if the assertion of the form: For every $T$ and for every $\rho(x, y) \in C$, where $C$ is a cone of the set $G$ of all cones, the following fact in the form

$$
\mathfrak{C}(x, y \in X, \preccurlyeq, \oplus, T, \rho) \text { implies } T \text { has a fixed point }
$$

is a theorem. (We recall that a statement is Local Principle of Transpose if (B) and (BA) hold only via term of the form $x \in X$ ).

In connection with the preceding statement if facts (B) and (BA) to substitute with the following facts in the forms as

$$
\mathfrak{C}(x, y \in X, \leq,+, T, \rho) \quad \text { implies } \mathfrak{M}(T),
$$

and

$$
\mathfrak{C}(x, y \in X, \preccurlyeq, \oplus, T, \rho) \quad \text { implies } \mathfrak{M}(T),
$$

respectively, where the property $\mathfrak{M}(T)$ denotes all conclusions of the Banach contraction principle: 1) $T$ has a unique fixed point $\xi \in X, 2$ ) $x_{n}=T^{n}(x) \rightarrow \xi$ for every $x \in X$, and 3) there exists an estimate of the rapidity of convergence; then Theorem 47 also to remain holds.

Consequences of the principles of transpose. Let $X:=(X, \rho)$ be a cone metric space and $\left\{x_{n}\right\}_{n \in \mathbb{N}}$ be a sequence in $X$. Let $x \in X$, if for every $c \in E$ with $\theta \preccurlyeq c$ there is $n_{0} \in \mathbb{N}$ such that $\rho\left[x_{n}, x\right] \preccurlyeq c$ for every $n \geq n_{0}$, then $\left\{x_{n}\right\}_{n \in \mathbb{N}}$ is said to be convergent and it converges to $x$, i.e., $x$ is limit of $\left\{x_{n}\right\}_{n \in \mathbb{N}}$. If for any $c \in E$ with $\theta \preccurlyeq c$, there is $n_{0} \in \mathbb{N}$ such that, $\rho\left[x_{n}, x_{m}\right] \preccurlyeq c$ for all $n, m \geq n_{0}$, then $\left\{x_{n}\right\}_{n \in \mathbb{N}}$ is called a Cauchy sequence in $X$. If every Cauchy sequence is convergent in $X$, then $X$ is called a complete cone metric space.

There exist several applications of the preceding principles of transpose. In the fixed point theory, theorems of the forms (A), (E) and (B) are usually proved first.

However, theorems of the forms (TA), (R) and (BA) are more general (in the sense of sufficiency), so the proofs of the theorems are usually similar. Using our principles of transpose, we are able to state at once the theorems (A) and (TA), (E) and (R) i.e., (B) and (BA) depending which of the theorems is wanted. We shall illustrate the preceding principles of transpose with the several examples. 
In this sense we give some illustrations of the preceding Theorem 46 (Principle of Transpose) for the intervals of the form $[a, b)$ for $\prec b$ in the form of upper spring ordered transversal spaces:

Example 1. (K u r e p a [65]). As a first example of upper spring ordered spaces we obtain so-called pseudodistantial space by Đ. Kurepa with the nonnumerical transverse. In this case the nonnumerical transverse is given in the partially ordered set $[a, b)$, where the element $a$ in the partially ordered set $G$ of the following form: $[a, b)=(G,+, \preccurlyeq)$ is a linearly ordered abelian groups with cofinality $\operatorname{cof}(G)=w_{\mu}$ at he identity element $a=\theta \in G$ (which means that $a$ is the infimum of a strictly decreasing $\tau$-sequnce). An $\tau$ metric on $X$ is a function $\rho: X \times X \rightarrow[a, b)=G$ which satisfies all the metric axioms (i.e., $\rho[x, y]=\theta=a$ if and only if $x=y, \rho[x, y]=\rho[y, x]$, and $\rho[x, y] \preccurlyeq \rho[x, z]+\rho[z, y])$. For this see: Collat z [27], $\mathrm{C}$ a m m a r o t oK o č in a c [67] and S ikorski [66].

Example 2. (Kantorovitch's lineal, $\mathrm{K}$ ant or ovitch [68]). Let $K$ be a real linear space and $K$ be a $K$-lineal, by L. V. Kantorovitch. In this sense, $K$ is a $\mathbf{K}$-lineal if there exists an element $\theta \in K$ as a neutral element such that $\theta \prec x$ for all $x \in K$, and if the partially ordering $\preccurlyeq$ is defined with $x \preccurlyeq y$ if and only if $x-y \succcurlyeq \theta$ for all $x, y \in K$ such that the following hold:

(i) $x \succ \theta, y \succ \theta$ implies $x \neq \theta, x+y \succ \theta$, and $x y \succ \theta$;

(ii) there exists $\sup \{x, y\}$ for two arbitrary elements $x, y \in K$.

In this case, $[a, b)=K$ with nonnumerical upper transversal $\rho: X \times X \rightarrow$ $K$ as an element in $K$.

Example 3. (M-sets, Ta s k ovi ć [37]). Let $\mathcal{O}$ be a partially ordered set by the relation $\preccurlyeq$ such that there exists $\theta \in \mathcal{O}$ with the property: a) $\theta \preccurlyeq u$ for every $u \in \mathcal{O}$. Also, 2): for every nonincreasing sequence $\left\{u_{n}\right\}_{n \in \mathbb{N}}$ there exists the unique element $u \in \mathcal{O}$ called the limit of $\left\{u_{n}\right\}_{n \in \mathbb{N}}$ all signed by $u=\lim _{n \rightarrow \infty} u_{n}$ (alternative designation $u_{n} \downarrow u$ ) such that: $u_{n}=u$ (for $n \in \mathbb{N}$ ) implies $u_{n} \downarrow u$; if $u_{n} \downarrow u, v_{n} \downarrow v, u_{n} \preccurlyeq v_{n}$, then $u \preccurlyeq v$; and the limit of $\left\{u_{n}\right\}_{n \in \mathbb{N}}$ is invariant with respect to the initial conditions. The partially ordered set $\mathcal{O}$ with the preceding properties we call the M-set.

We notice that the property 2 ) is specially realized if in $\mathcal{O}$ is introduced the usual ordered topological structure and eachy subset of $\mathcal{O}$ from the upper side bounded has its supremum, the term of limit having its standard meaning.

If the set $[a, b)=\mathcal{O}$ where $a=\theta$ we obtain an upper nonnumerical transverse $\rho: X \times X \rightarrow \mathcal{O}$ as an element in $[a, b)$. It is evident that $\rho$ satisfy all conditions of the upper transversal ordered spring transverse!!

Example 4. (The cone metrical spaces, $\mathrm{K} \mathrm{u} \mathrm{r} \mathrm{e} \mathrm{p} \mathrm{a} \mathrm{[65]).} \mathrm{We} \mathrm{begin} \mathrm{by}$ introducing a structure ot the ordered Banach space and define a cone metric space. Let $E$ be a real Banach space and $P$ a subset of $E$. In this case $P$ is called a cone if: $P$ is a closed, nonempty set and $P \neq\{0\}, a x+b y \in P$ for all $a, b \in \mathbb{R}(a, b \geq 0)$ and $x, y \in P$, and $x \in P$ such that $-x \in P$ implies $x=0$. In this case $a=0 \in[a, b)$. 
Given a cone $P \subset E$ we define a partially ordering $\preccurlyeq$ with respect to $P$ by $x \preccurlyeq y$ if and only if $y-x \in P$. We shall write $x \prec y$ iff $x \preccurlyeq y$ and $x \neq y$. Also, we shall write $x \ll y$ iff $y-x \in \operatorname{int} P$, where int $P$ denotes the interior of $P$.

In this sense, suppose that the mapping $\rho: X \times X \rightarrow E$ has all the metric axioms as in the papers: Kurepa [65], Collatz [27] and Sikorski [66]. Thus $\rho$ satisfis all the axioms of the upper transversal ordered spring spaces (as and all axioms of Kurepa's pseudodistantial spaces, see: K u r e p a [65]).

\section{ACKNOWLEDGEMENT}

I am indebted to VMA (= Military Medical Academy of Serbia Army) for recovery of health and remedial treatment. In this context I am indebted to: Professor Dr Zoran Anđelković, Professor Dr Miroljub Drašković, Professor Dr Zoran Hajduković, Professor Dr Jevta Kozarski, Dr Vladislav Krstić, Dr Jelena Karajovi'c, Dr Tomislav Mladenovi'c and Professor Dr Marjan Novaković.

Taking one consideration with another, this paper is originate by studying preceding problems for during my stay at VMA-Beograd!

\section{REFERENCES}

[1] Banach, Stefan. Sur les opérations dans les ensembles abstraits et leur application aux équations intégrales, Fund. Math., 3 (1922), 133-181.

[2] Constantin, Adrian. Coincidence point theorems for multivalued contraction mappings, Math. Japonica, 36 (1991), 925-933.

[3] Dugundji, J., and Granas, Andrzej, Weakly contractive maps and elementary domain invariance theorem, Bull. Greek Math. Soc., 19 (1978), 141-151.

[4] Dugundji, J., and Granas, Andrzej, Fixed point theory, Vol. I., Monografie Mathematyczne 61, Polska Acad. Nauk, 1982, Warsaw, 209 p.p.

[5] Browder, Felix, E. On the convergence of successive approximations for nonlinear functional equations, Indagationes, Math., 30 (1968), 27-35.

[6] Browder, Felix, E. On a theorem of Caristi and Kirk, Proc. Seminar of Fixed Point Theory and its Appl., Dalhousie University, June 1975, 23-27.

[7] Browder, Felix, E. Remarks on fixed point theorems of contractive type, Nonlinear Analysis, 3 (1979), 657-661.

[8] Boyd, D.W., and Wong, J.S.W. On nonlinear contractions, Proc. Amer. Math. Soc., 20 (1969), 458-464.

[9] Caristi, J. Fixed point theorems for mappings satisfying inwardness conditions, Trans. Amer. Math.Soc., 215(1976), 241-251.

[10] Caristi, J., and Kirk, W. A. Geometric fixed point theory and inwardness conditions, Proc. Conf. on Geometry of Metric and Linear Spaces, Michigan, 1974, Lecture Notes in Mathematics 490, Springer-Verlag, 1975, 75-83.

[11] Hicks, T. L., and Rhoades, B. E., A Banach fixed point theorem, Math. Japonica, 24 (1979), 327-330. 
[12] Hicks, T. L., and Rhoades, B. E., Fixed point theorems for d-complete topological spaces-II, Math. Japonica, 37 (1992), 847-853.

[13] Fisher, B., Mappings on a metric space, Boll. U. M. I., 12 (1975), 147-151.

[14] Kirk, W. A. Caristi's fixed point theorem and metric convexity, Collog. Math., 36 (1976), 81-86.

[15] Park, Sehie. Equivalent formulations of Ekeland's variational principle and their applications, MSRI-Korea Publ., 1 (1986), 55-68.

[16] Krasnoselskij, M. A., and Stečenko, V. Teoria ecuatilor cu operatori concavi, Sib. Math. J., 10 (1969), 565-572.

[17] Krasnoselskij, M. A. Two remarks on the method of succesive approximations, Uspeh Mat. Nauk, 10 (1955), 123-127, (Russian).

[18] Krasnoselskij, M. et al. Näherungsverfahren zur Lösung von Operatorgleichungen, Akademie-Verlag, Berlin, 1973.

[19] Kiventidis, Thomas. On fixed points in Hausdorff spaces, Indian J. pure appl. Math., 16 (1985), 1420-1424.

[20] Rus, Ioan. A. Fixed point structures, Mathematica (Cluj-Napoca), 28 (1986), 59-64.

[21] Iséki, Kiyoshi, On Banach theorem of contraction mappings, Proc. Japan Acad, 41 (1967), 287-289.

[22] Iséki, Kiyoshi, Some fixed point theorems in metric spaces, Math. Jap. 20 (1975), 101-110.

[23] Walter, Wolfgang. Remark on a paper by F. Browder about contraction, Nonlinear Analysis, 4 (1981), 21-25.

[24] Daneš, J. On densifying and related mappings and their applications in nonlinear functional analysis. In: Theory of Nonlinear Operators, J. Nečas et al., [ed.]. Akademie-Verlag, Berlin 1972, 15-56.

[25] Iséki, Kiyoshi. Fixed point theorems in 2-metric spaces, Math. Sem. Notes, 3 (1975), 133-136.

[26] Meir, A., and Keeler, E. A theorem on contraction mappings, J. Math. Anal. and Appl., 28 (1969), 326-329.

[27] Collatz, Lothar. Funktionalanalysis und Numerische Mathematik, Springer-Verlag, Berlin, 1964.

[28] Istrăţescu, Vasile. I. Fixed Point Theory, Reidel Publishing Company, Dordrecht/Boston/London, 1981, 466 pages.

[29] Massa, Silvio. Generalized contractions in metric spaces, Boll. Unione Mat. Ital., 10 (1974), 689-694.

[30] Kurepa, Đuro, R. Some cases in the fixed point theory, Topology and its Applications, Budva, 1972, 144-153.

[31] Miczko, A., and Palczewski, B. Common fixed points of contractive type mappings in a 2-metric space, Math. Nachr., 124 (1985), 341-355.

[32] Kwapisz, M. Some generalization of an abstract contraction mapping principle, Nonlinear Anal. Theory, Meth. Appl., 3 (1979), 293-302.

[33] Wong, Chi. Song. Maps of contractive type, Proc. Seminar of Fixed Point Theory and its Appl., Dalhousie University, June 1975, 197-207. 
[34] M. R. Tasković: A characterization of the class of contraction type mappings, Kobe J. Math., 2 (1985), 45-55.

[35] M. R. Tasković: Some results in the fixed point theory-II, Publ. Inst. Math., 41 (1980), 249-258.

[36] M. R. Tasković: A monotone principle of fixed points, Proc. Amer. Math. Soc., 94 (1985), 427-n432.

[37] M. R. Tasković: Osnove teorije fiksne tačke, Mat. Biblioteka, 50 (Beograd 1986), p. p. 272. English summary: Fundamental elements of the fixed point theory, 268-271.

[38] M. R. Tasković: Some new principles in fixed point theory, Math. Japonica, 35 (1990), 645-n666.

[39] M. R. Tasković: Nonlinear Functional Analysis, (Fundamental Elements of Theory). First Book: Monographs, Zavod za udžbenike i nastavna sredstva, Beograd 1993, 792 p.p., (Serbo-Croation). English summary: Comments only new main results of this book, Vol. 1 (1993), 713-752.

[40] M. R. Tasković: Nonlinear Functional Analysis, Second Book, Monographs - Global Convex Analysis: General convexity, Variational methods and Optimization, Zavod za udžbenike i nastavna sredstva and Vojnoizdavački zavod, Beograd 2001, (In Serbian), 1223 pages.

[41] M. R. Tasković: A directly extension of Caristi fixed point theorem, Math. Moravica, 1 (1997), 105-108.

[42] M. R. Tasković: New geometric fixed point theorems, Math. Moravica, 2 (1998), 143-148.

[43] M. R. Tasković: Extension of theorems by Krasnoselskij, Stečenko, Dugundji, Granas, Kiventidis, Romaguera, Caristi and Kirk, Math. Moravica, 6 (2002), 109-118.

[44] M. R. Tasković: General expansion mappings on topological spaces, Scientiae Mathematicae Japonicae, No.1, 54 (2001), 61-67,: e4, 497-503.

[45] M. R. Tasković: Fixed points and apices on arbitrary sets, Math. Moravica, 5 (2001), 111-118.

[46] M. R. Tasković: Transversal intervally spaces, Math. Moravica, 7 (2003), 91-106.

[47] M. R. Tasković: Furcate points and lower BCS-convergence in the fixed point theory, First International Videoconference of Math. Science - Fixed Point Theory, Japanese Assoc. Math. Sciences, Osaka, Japan, 2003, 10 p.p.

[48] M. R. Tasković: Fixed points on transversal edges spaces, Math. Moravica, 7 (2003), 175-186.

[49] M. R. Tasković: Survey on transversal normed spaces, Math. Moravica, 7 (2003), 153-174.

[50] M. R. Tasković: Geometric Fixed Point Theorems on Transversal Spaces, Math. Moravica, 8-2 (2004), 29-52.

[51] M. R. Tasković: Theory of transversal point, spaces and forks, Monographs of a new mathematical theory, VIZ-Beograd 2005, (In Serbian), 1054 pages. English summary: 1001-1022.

[52] M. R. Tasković: Some theorems on fixed point and its applications, Ann. Soc. Math. Polon. Ser. I, Comment. Math. Prace. Math., 24 (1984), 323-334. 
[53] M. R. Tasković: Some results in the fixed point theory, Publ. Inst. Math., 34 (1976), 231-242.

[54] M. R. Tasković: Transversal ordered interval and edges spaces, fixed points and applications, Math. Moravica, 13-1 (2009), 49-75.

[55] M. R. Tasković: A generalization of Banach's contraction principle, Publ. Inst. Math. Beograd, 37 (1978), 179-191.

[56] M. R. Tasković: On some mappings of contraction type, Abstracts, 4 th., Balkan Math. Congress, Istanbul, 1971, p. 103.

[57] Romaguera, S. Fixed point theorems for mappings in complete quasimetric spaces, Anal. Stii. Universit. "Al. I. Cuza" Iaşi, 39 (1993), 159-164.

[58] Akkouchi, M. On a result of W. A. Kirk and L. M. Saliga, J. of Comput. Appl. Math., 142 (2002), 445-448.

[59] Kirk, W. A., and Saliga, L. M. Some results on existence and approximation in metric fixed point theory, J. of Comput. and Appl. Math., 113 (2000), 141-152.

[60] Wang, S. Z., Gao, Z. M., Li, B. Y., and Iséki, K. Some fixed point theorems on expansion mappings, Math. Japonica, 29 (1984), 631-636.

[61] Daffer, P. Z., and Kaneko, H. On expansive mappings, Math. Japonica, 37 (1992), 733-735.

[62] K. Kim, and K. H. Leem: Note on common fixed point theorems in metric spaces, Comm. Korean Math. Soc., 11 (1996), 109-115.

[63] K. Kim, T. H, Kim, K. H. Leem, and J. S. Ume: Common fixed point theorems relating to the diameter of orbits, Math. Japonica, 47 (1998), 103-108.

[64] Ohta, M., and Nikaido, G. Remarks on fixed point theorems in complete metric spaces, Math. Japonica, 39 (1994), 287-290.

[65] Kurepa, Đuro, R. Tableaux ramifiés d'ensembles. Espaces pseudo-distanciés, C. R. Acad. Sci. Paris, 198 (1934), 1563-1565.

[66] Sikorski, R. Remarks on some topological spaces of high power, Fund. Math., 37 (1950), 125-136.

[67] Cammaroto, F., and Kočinac, Lj. Some Results on $\omega_{\mu}$-Metrizable and Related Spaces, Boll. U. M. I., 7-B (1993), 607-629.

[68] Kantorovitch, L. V. The method of successive approximations for functional equations, Acta Math., 71 (1939), 63-97.

\author{
Milan R. Tasković \\ Faculty of Mathematics \\ P.O. Box 550 \\ 11000 BEOGRAD \\ SERBIA \\ Home address: \\ Milan R. Tasković \\ Nehruova 236 \\ 11070 BELGRADE \\ SERBIA \\ E-mail address: andreja@predrag.us
}

